Article

\title{
Infrared Investigations of the Neutral-Ionic Phase Transition in TTF-CA and Its Dynamics
}

\author{
Martin Dressel * and Tobias Peterseim \\ 1. Physikalisches Institut, Universität Stuttgart, Pfaffenwaldring 57, D-70550 Stuttgart, Germany; \\ tpeterseim@gmx.de \\ * Correspondence: dressel@pi1.physik.uni-stuttgart.de; Tel.: +49-711-6856-4946; Fax: +49-711-6856-4886 \\ Academic Editors: Anna Painelli and Alberto Girlando \\ Received: 30 November 2016; Accepted: 3 January 2017; Published: 7 January 2017
}

\begin{abstract}
The neutral-ionic phase transition in TTF-CA was investigated by steady-state and time-resolved infrared spectroscopy. We describe the growth of high-quality single crystals and their characterization. Extended theoretical calculations were performed in order to obtain the band structure, the molecular vibrational modes and the optical spectra along all crystallographic axes. The theoretical results are compared to polarization-dependent infrared reflection experiments. The temperature-dependent optical conductivity is discussed in detail. We study the photo-induced phase transition in the vicinity of thermally-induced neutral-ionic transition. The observed temporal dynamics of the photo-induced states is attributed to the random-walk of neutral-ionic domain walls. We simulate the random-walk annihilation process of domain walls on a one-dimensional chain.
\end{abstract}

Keywords: charge-transfer salts; TTF-CA; neutral-ionic phase transition; crystal growth; characterization; steady-state and transient infrared spectroscopy; vibrational spectroscopy; light-induced phase transition; domain-wall motion; random-walk simulations

PACS: 77.84.Jd; 78.30.-j; 63.20.-e; 77.80.Dj; 77.80.Fm

\section{Introduction}

TTF-CA consists of the two organic molecules, tetrathiavulvalene (TTF, $\left.\mathrm{C}_{6} \mathrm{~S}_{4} \mathrm{H}_{4}\right)$ and chloranil $\left(\mathrm{CA}, \mathrm{C}_{6} \mathrm{Cl}_{4} \mathrm{O}_{2}\right.$ ), whose molecular structure is depicted in Figure 1a. It was first synthesized in 1979 [1] with the main goal to create an organic compound with a high electric conductivity that can compete with conventional metals. At present, several synthesis methods exist, which are described in detail in Section 2. In all cases, the stoichiometric ratio of the starting materials is 1:1. At room temperature, the monoclinic unit cell contains two equivalent TTF and CA molecules. The molecules TTF and CA are alternating and equally spaced along the $a$-direction, as depicted in Figure $1 \mathrm{c}, \mathrm{d}$. The CA is the acceptor molecule (A) while TTF is the electron donator (D). Hence, charge transfer takes place between them. Both the TTF and CA molecules are arranged parallel to their molecular plane in the unit cell with a distance of $3.70 \AA$ [2]. Thereby, they are shifted and rotated slightly towards each other, leading to a maximum overlap of the molecular orbitals. First studies on the crystal structure and the bond lengths of the $\mathrm{C}=\mathrm{C}$ double bond of TTF and CA as well as the $\mathrm{C}=\mathrm{O}$ bonds in CA reveal that the molecules are almost neutral at room temperature caused by a small charge transfer between TTF and CA [3] that can be attributed to the large intermolecular distance of $3.70 \AA$ [2]. Indeed, infrared measurements show that the charge transfer is $\rho=0.2 e[4,5]$ at ambient conditions. Therefore, this state is referred to as the neutral phase. Due to the small charge transfer, the crystals glimmer greenish and transparent because the total absorption and reflection spectrum is composed of the single spectrum of the quasi-neutral $\mathrm{TTF}^{0}$ and $\mathrm{CA}^{0}$ molecules whose electronic excitations in the visible range are blue-shifted. For that reason, it is often called the green phase in literature. The color 
can be utilized to check the quality of the crystals (see Figure 5). Besides the green phase, a so-called black phase exists which exhibits different physical properties, for instance the absence of the neutral to ionic phase transition.
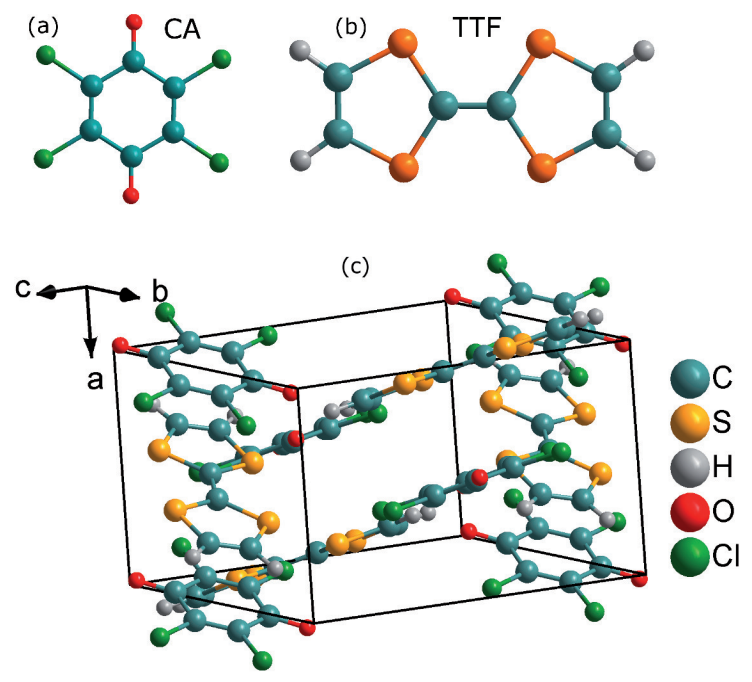

(b)

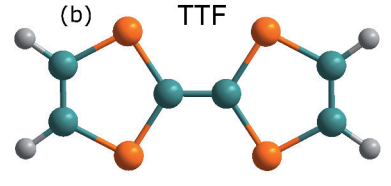

(c)
Figure 1. (a) Chloranil molecule (CA, $\mathrm{C}_{6} \mathrm{Cl}_{4} \mathrm{O}_{2}$, color of the material: yellow); (b) Tetrathiafulvalene (TTF, $\mathrm{C}_{6} \mathrm{~S}_{4} \mathrm{H}_{4}$, color of the solid: orange); (c) Monoclinic unit cell of TTF-CA at room temperature. The TTF and CA molecules are ordered along the crystallographic $a$-axis [2]. Due to the crystal symmetry and the position of the molecules, a unit cell contains two equivalent TTF and CA molecules; (d) At room temperature, the space group of the unit cell is $\mathrm{P} 2_{1} / \mathrm{n}$ and the CA and TTF molecules are stacked equally spaced along the $a$-axis. A further stack is located at $z=c=0.5$, respectively, at which the TTF-CA pairs are tilted opposite to the $a$-axis. Lower right: in the ionic phase, the TTF and CA molecules dimerize along the $a$-direction. By the charge transfer of about $\rho=0.6 e$, electric dipoles are formed along the stacking direction, resulting in a loss of the two-fold screw axis $2_{1}[6,7]$.

Upon cooling, the charge transfer increases slightly from $0.2 e$ to $0.3 e[2,4,5,8]$. At $T_{\mathrm{NI}}=82 \mathrm{~K}$, a phase transition occurs, at which the space group is lowered from $\mathrm{P} 2_{1} / \mathrm{n}$ to $\mathrm{Pn}$ as identified by structure-resolving techniques. Upon the transition, two symmetry operations are lost-the point inversion and the screw rotation along the $b$-direction-as deflected in the abrupt appearing of the $(0 k 0)$ reflections plotted in Figure 2a. Due to thermal contraction, the unit cell shrinks and the cell parameters $b$ and $c$ jump to lower values at the phase transition. Furthermore, in the low temperature phase, the TTF and CA molecules dimerize along the $a$-axis: the initial distance of $3.70 \AA$ decreases to $3.504 \AA$ and $3.685 \AA$ [2], as it is depicted in Figure 1c, respectively. Also, the ionicity $\rho$ increases from $0.3 e$ to about $0.6 e[2,4,5,8]$, which was consistently determined by infrared, Raman and X-ray studies. The strong dimerization and the charge transfer lead to the creation of strong electric dipoles between the TTF and CA molecules. This was demonstrated by dielectric measurements showing a sharp feature in $\epsilon_{1}$ at the transition temperature $T_{\mathrm{NI}}$. In Figure $2 \mathrm{~b}$, the real part $\epsilon_{1}$ of the complex dielectric function is displayed versus temperature for two frequencies, $f=100 \mathrm{~Hz}$ and $100 \mathrm{kHz}$. At the transition temperature $T_{\mathrm{NI}}, \epsilon_{1}(T)$ rises steeply and diverges for both frequencies. This behavior corresponds to a transition from a paraelectric to a ferroelectric state, where $\epsilon_{1}$ follows a Curie-Weiss law above the critical temperature $T_{\mathrm{NI}}$. The feature between $T=100$ and $200 \mathrm{~K}$ for $f=100 \mathrm{~Hz}$ is attributed to solitons and neutral-ionic domains walls, which will be described in detail in the subsequent Sections 5 and 6.

In Figure 2c, the temperature-dependent behavior of the specific heat $C_{p}(T)$ is illustrated. Similar to $\epsilon_{1}(T)$, a pronounced divergency of $C_{p}(T)$ is observed at the phase transition, giving evidence for a first-order transition. 


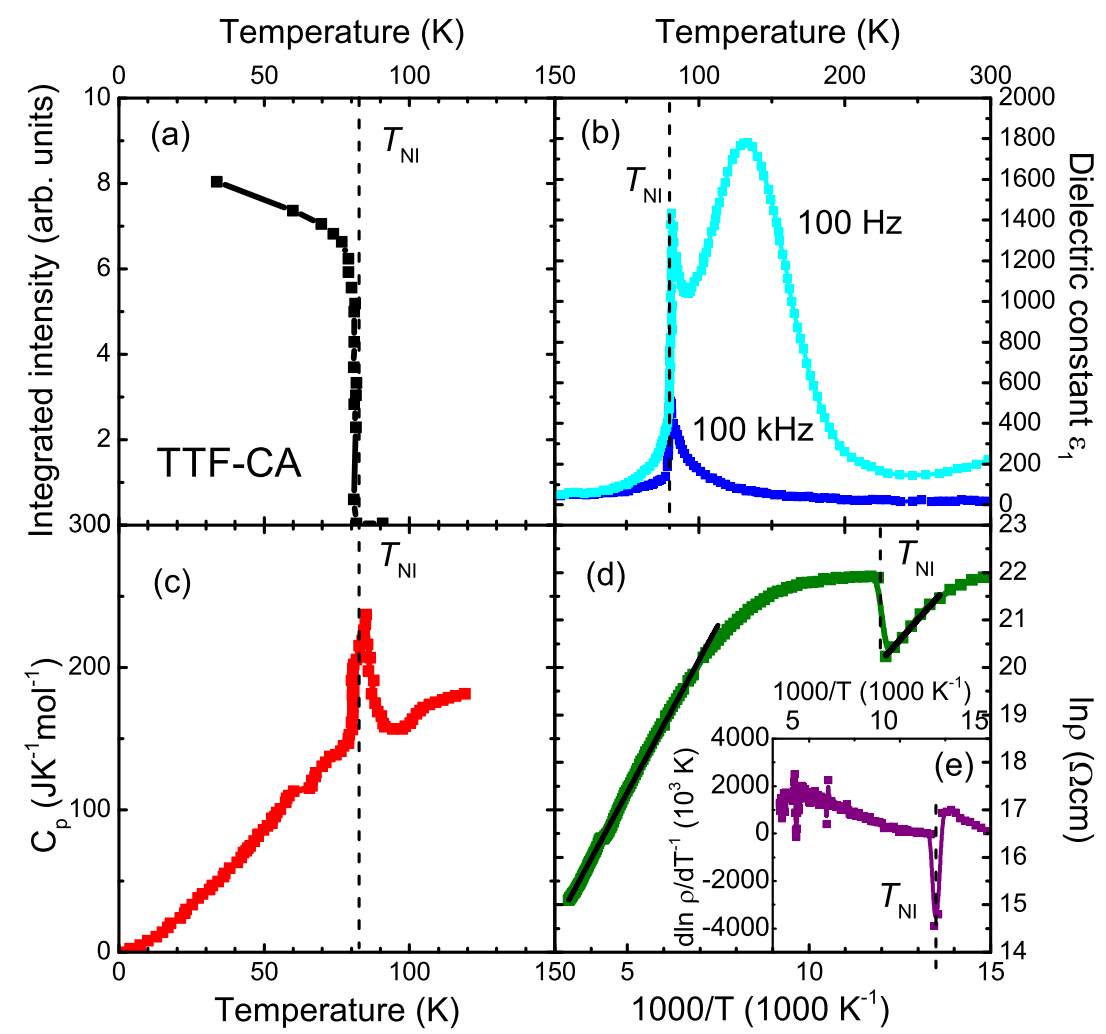

Figure 2. (a) Temperature-dependent (0k0)-reflection of TTF-CA [2], occurring after the loss of the twofold screw axis which is caused by the break of symmetry and the structural phase transition; (b) Temperature evolution of the real part $\epsilon_{1}$ of the dielectric function, recorded at a frequency of $100 \mathrm{~Hz}$ (light blue) and $100 \mathrm{kHz}$ (dark blue). $\epsilon_{1}$ diverges for all frequencies at the critical temperature $T_{\mathrm{NI}}$ and marks a first-order para- to ferroelectric transition [9]. The feature, appearing between $T=100$ and $200 \mathrm{~K}$ for $100 \mathrm{~Hz}$, is attributed to the excitation of neutral-ionic domain walls; (c) At the phase transition, the specific heat capacity $C_{p}$ of TTF-CA diverges similar to $\epsilon_{1}$, indicating a first-order phase transition [10]; (d) Arrhenius plot of the resistivity $\rho_{d c}$ (green) of TTF-CA along the stacking direction. Above the transition, it behaves as a classic band insulator with an activation energy of $\Delta=0.12 \mathrm{eV}$.

Figure $2 \mathrm{~d}$ displays the resistivity $\rho_{d c}$ along the stacking direction of a TTF-CA single crystal. The specific resistivity in the neutral phase is $\rho_{d c}=3 \times 10^{6} \Omega \mathrm{cm}$ indicating an insulating behavior at ambient conditions. Previous dc-measurements from Mitani et al. [11] yield values of $\rho_{d c}$ between $10^{5}$ and $10^{6} \Omega \mathrm{cm}$. Upon cooling, the resistivity follows an Arrhenius development $\rho_{d c}(T)=\rho_{0} \exp \{\Delta / T\}$, at which $\Delta$ is the temperature-independent activation energy, or energy gap in a semiconductor picture, respectively. In the neutral phase, the activation energy is $0.12 \mathrm{eV}$ (corresponding to $1276 \mathrm{~K}$ ), which is close to the literature values lying between $0.095 \mathrm{eV}$ and $0.065 \mathrm{eV}[9,11,12]$. In the inset (e) of Figure 2, the derivative of the logarithmic resistivity is presented, in order to illustrate the variations of the slope upon cooling. At the transition to the ionic phase, the resistivity $\rho_{d c}(T)$ is reduced by one order of magnitude, but it increases again afterwards. Below the transition $T_{\mathrm{NI}}$ the activation energy $\Delta$ is only $0.065 \mathrm{eV}$ and agrees excellently with literature values.

In order to better understand the neutral-ionic phase transition, let us consider the total energy $[13,14]$

$$
E_{\text {tot }}(\rho)=(I-A) \rho-\alpha\left\langle\frac{e^{2}}{d_{\text {Dim }}}\right\rangle \rho^{2}
$$

of a donor-acceptor (D-A) crystal, such as TTF-CA. Obviously, it mainly depends on the ionicity $\rho$. Here, the first term $I-A$ describes the energy necessary to ionize a D-A pair, at which $I$ is the 
ionization energy of $\mathrm{D}$, and $A$ is the electron affinity of $\mathrm{A}$. It competes with the Madelung energy $\alpha\left\langle\frac{e^{2}}{d_{\operatorname{Dim}}}\right\rangle$ set free in case the lattice becomes ionic. $d$ is the distance between the acceptor and the donor molecule and varies with temperature; $e$ the electron charge and $\alpha$ the Madelung constant. $E_{\text {tot }}(\rho)$ is minimal for the case $\rho=0$ if $(I-A)>\alpha\left\langle\frac{e^{2}}{d_{\text {Dim }}}\right\rangle$, and for the case $\rho=1$ if $(I-A)<\alpha\left\langle\frac{e^{2}}{d_{\text {Dim }}}\right\rangle$. When $(I-A)<\alpha\left\langle\frac{e^{2}}{d_{\operatorname{Dim}}}\right\rangle$, a transition takes place from a neutral to ionic phase. This means that in the D-A crystal, the terms $I-A$ and $\alpha\left\langle\frac{e^{2}}{d_{\text {Dim }}}\right\rangle$ compete with each other. In the case of TTF-CA, the ionization energy of TTF is $I=6.2-6.8 \mathrm{eV}$ [15-18] and the electron affinity of CA is $A=2.8 \mathrm{eV}$ [19], resulting in $I-A=3.7 \mathrm{eV}$ with $I=6.5 \mathrm{eV}$ for TTF. Tanaka et al. [20] calculated a Madelung energy of $\alpha\left\langle\frac{e^{2}}{d_{\operatorname{Dim}}}\right\rangle=3.59 \mathrm{eV}$ for TTF-CA, leading to an energy difference between the Madelung energy and $I-A$ of about $0.1 \mathrm{eV}$. Energetic considerations hence confirm that under ambient conditions, TTF-CA is supposed to be in the neutral phase. As seen from Equation (1), the Madelung energy is a function of the intermolecular distance $d_{\text {Dim }}$ between $\mathrm{D}$ and $\mathrm{A}$. Thus, varying the distance by thermal contraction upon cooling or by hydrostatic pressure, the Madelung energy increases and becomes larger than $I-A$. This leads to a transition from the neutral to the ionic phase occurring at $T_{\mathrm{NI}}=81.5 \mathrm{~K}$ or at approximately $9 \mathrm{kbar}$ for room temperature [21].

Due to the thermal shrinking of the unit cell parameter $a$, as listed in Table 1, the molecule distance $d_{\text {Dim }}$ decreases and induces the phase transition at $T_{\mathrm{NI}}=81.5 \mathrm{~K}$. According to the above energy considerations, this transition is possible even at room temperature by applying hydrostatic pressure. This was first shown by Mitani et al. [11] measuring the electrical transport under pressure. They found that a pressure of $p=11$ kbar induces the ionic phase at $T=295 \mathrm{~K}$; with decreasing temperature, less pressure is necessary to trigger the transition. Recently, Dengl et al. obtained the ionicity of TTF-CA as a function of temperature for different pressure values. While there is a clear jump of $\rho(T)$ at $T_{\mathrm{NI}}=81 \mathrm{~K}$ at ambient pressure, the step becomes reduced and the transition smoother as pressure increases. Above $9 \mathrm{kbar}$, the ionicity basically reaches the value of the ionic phase already at room temperature [21].

Table 1. Unit cell parameter TTF-CA for $300 \mathrm{~K}$ and $40 \mathrm{~K}$. The data were taken from Mazerle et al. and LeCointe et al. [2,3].

\begin{tabular}{ccc}
\hline Parameter & TTF-CA (300 K) [3] & TTF-CA (40 K) [2] \\
\hline$a(\AA)$ & 7.41 & 7.19 \\
$b(\AA)$ & 7.621 & 7.54 \\
$c(\AA)$ & 14.571 & 14.44 \\
$\alpha\left({ }^{\circ}\right)$ & 90 & 90 \\
$\beta\left({ }^{\circ}\right)$ & 99.2 & 98.6 \\
$\gamma\left({ }^{\circ}\right)$ & 90 & 90 \\
$V\left(\AA^{3}\right)$ & 812.35 & 774.03 \\
$Z$ & 2 & 2 \\
$M\left(\mathrm{~g} \cdot \mathrm{mol}^{-1}\right)$ & 900.74 & 900.74 \\
$\rho_{m}\left(\mathrm{~g} \cdot \mathrm{cm}^{-3}\right)$ & 1.82 & 1.93 \\
Space group & $\mathrm{P} 2_{1} / \mathrm{n}$ & $\mathrm{Pn}$ \\
\hline
\end{tabular}

Based on transport measurements, similar observations are reported [11]: The down and up turn of the conductivity at ambient pressure (see Figure $2 b$ ) becomes broader and shifts to higher temperatures. The conductivity increases exponentially as it does with the pressure. The strong increase of the conductivity is attributed to the increase of the number of neutral-ionic domain walls (NIDW) in the neutral phase. This is also valid for the ionic phase. Model calculations [22-24] reveal that NIDW can also be excited at room temperature and their concentration decreases with decreasing temperature. However, the simulations confirm the influence of these NIDW on the transport properties and on the pressure-induced phase transition. The NIDW are also responsible for the feature observed in the dielectric function above the phase transition [9], the electric transport [25] 
and the nonlinear conductivity [12]. Originally, this was predicted theoretically $[22,23]$ and then confirmed quantitatively by Soos and Painelli [24], by taking into account the interaction of the lattice and the molecular vibrations with the electronic system. Additionally, several different energetically low-lying excitations exist in the neutral as well as in the ionic phase. Besides the excitation of NIDW, also polarons, spin-solitons [26,27], and charged solitons can be excited.

In Figure 3, the $p$ - $T$-phase diagram is depicted that is composed of several studies of different properties, i.e., electric transport [11], optical measurements [14,21,28-33], neutron scattering experiments and ${ }^{35} \mathrm{Cl} \mathrm{NQR}[34,35]$. The phase diagram reveals similarities to a solid-liquid-gas phase diagram with a triple point at $210 \mathrm{~K}$ and $500 \mathrm{MPa}$ where all three phases coexist. For low pressure and room temperature, TTF-CA resides in the neutral, non-dimerized phase $(\mathrm{N})$. For reduced temperatures $\left(T_{\mathrm{NI}}=81.5 \mathrm{~K}\right.$ and $\left.P_{\mathrm{NI}}=0 \mathrm{MPa}\right)$, a crossover into the ionic, dimerized phase $\left(\mathrm{I}_{\mathrm{ferro}}\right)$ occurs. At $T=300 \mathrm{~K}$, the ionic phase can be induced by applying external pressure of about $800 \mathrm{MPa}$. However, this state $\left(\mathrm{I}_{\text {para }}\right)$ is not dimerized A pressure of more than $1200 \mathrm{MPa}$ induces the transition to the dimerized, ferroelectric state. The physical nature of $\mathrm{I}_{\text {para }}$ is discussed controversially in literature. Studies on the crystal structure [2] indicate that it must be a paraelectric phase. Infrared data by Masino et al. [33] on single crystals of TTF-CA under hydrostatic pressure suggest that in the paraelectric phase two distinct ionic states are present. In contrast, most recent infrared investigations by Dengl et al. [21] on TTF-CA powder, examining the whole $P$ - $T$-space as depicted in Figure 4, give clear evidence that in this specific phase, there is no coexistence of two different ionic configurations. To finally make a conclusion on this "exotic" state, it will nevertheless be necessary to perform polarized infrared studies on single crystals under high pressure.

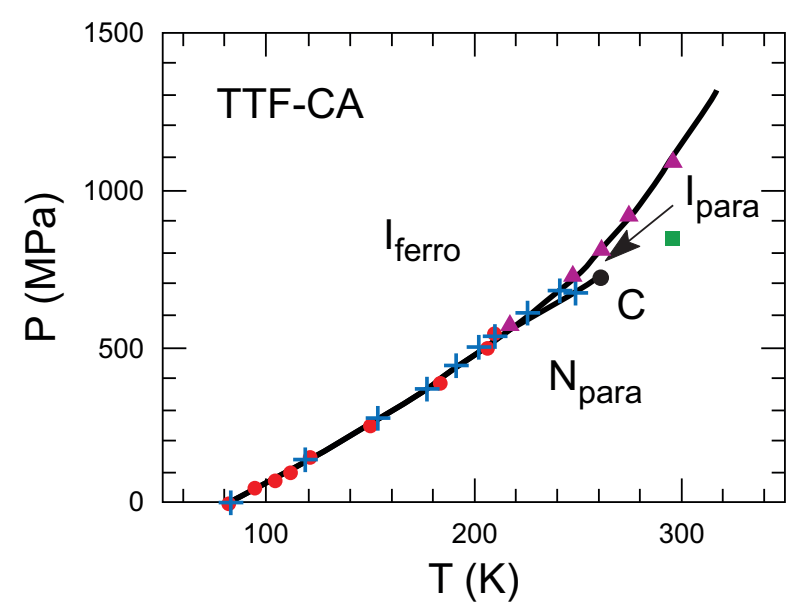

Figure 3. The $p$-T-phase diagram of TTF-CA exhibits, in a pressure range from 0 to $1500 \mathrm{MPa}$ and in a temperature range from 50 up to $350 \mathrm{~K}$, three different phases $\left(\mathrm{I}_{\text {ferro }}\right.$ : ferroelectric ionic phase, $\mathrm{I}_{\text {para }}$ : paraelectric ionic phase, $\mathrm{N}$ : neutral phase). The phase diagram resembles the characteristic of a solid-liquid-gas phase diagram. The red circles refer to neutron scattering and the blue plus signs to NQR results [34]; the violet triangle is obtained by vibrational spectroscopy [21,28,29]; the green square is extracted from transport measurements $[11,30]$. The black dot around $T_{\mathcal{C}}=260 \mathrm{~K}$ and a pressure slightly above $700 \mathrm{MPa}$ indicates the critical point labelled by C. Adapted from [6]. 

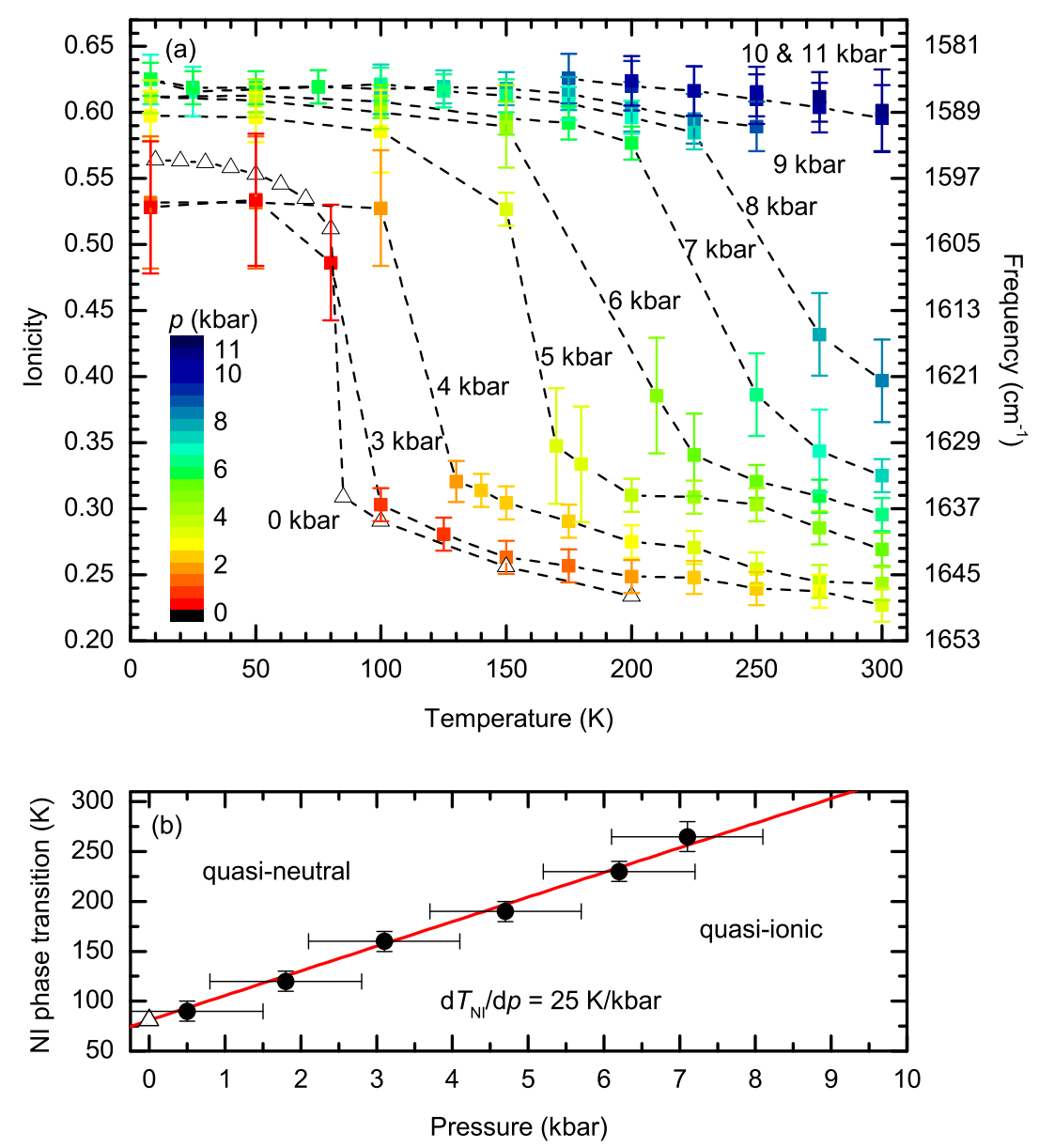

Figure 4. (a) Temperature dependence of the ionicity $\rho$ of TTF-CA at different hydrostatic pressure. The labels designate the value of pressure at room temperature, while the lines follow separate cooling runs from room temperature to $T=10 \mathrm{~K}$. The color of the points gives the corrected pressure as shown by the legend. The empty triangles represent separate ambient-pressure measurements on a single crystal. The quasi-ionic phase is defined by an ionicity $\rho>0.5$; (b) Phase diagram of TTF-CA as obtained from optical measurements of the vibrational peaks. The transition temperature under applied pressure (circle) increases by $25 \mathrm{~K} / \mathrm{kbar}$, starting from the $T_{\mathrm{NI}}=81 \mathrm{~K}$ at ambient pressure (triangle). Note that the pressure values shown here are corrected for the losses by thermal contraction (adapted from Ref. [21]).

\section{Crystal Growth}

Since crystals of TTF-CA and related compounds are not readily available commercially and produced only in very few selected laboratories, let us briefly report on our efforts at Universität Stuttgart that go back to the pioneer in this field, Norbert Karl. There are different crystal growth techniques available for the synthesis of charge-transfer salts, that area described in detail in Ref. [36-41] and references therein. The preferable methods to synthesize TTF-CA single crystals is the plate sublimation method or the slow cooling of an oversaturated solution of TTF and CA in an organic solvent.

For the cooling method, we use commercially available starting material, TTF and CA, from Sigma-Aldrich, München, Germany with a purity of $99.7 \%$ without further purification. Both materials were dissolved in a molar ratio of 1:1 at room temperature in $\mathrm{C}_{2} \mathrm{H}_{3} \mathrm{~N}$ (acetonitrile). When working under ambient conditions, the solvent evaporates rather fast; the solvent level decreases and small TTF-CA crystals crystallize at the walls of the glass recipient. The shape of the grown crystal is needle-like and very thin. The long axis points along the $a$-direction. These crystals have high quality 
but are unsuitable for infrared optical experiments due to their limited size and surface quality. Better results were achieved by decreasing the evaporation rate of the solvent. To that end, the glass vessel is placed in a refrigerator at $7{ }^{\circ} \mathrm{C}$. Thereby the crystallization process is slowed down and hence, the crystals become wider and longer. Furthermore, this method has the advantage that the whole process takes place in the absence of light. This way, any photochemical reaction during the synthesis is avoided that can disturb the growth process [36].

The plate sublimation technique was originally developed by N. Karl at the Universität Stuttgart: TTF and CA powder is heated and sublimated, it condenses at a colder plate and forms single crystals. The sublimation method provides naturally grown single crystals [37] of very high purity and quality. The TTF-CA crystals grow in trapezoidal shape in the $a$ - and $b$-direction as illustrated in Figure 5 and are large enough for optical measurements. In Figure 6, the setup of the plate sublimation is illustrated schematically; it was built according to Ref. [37]. The centerpieces are two cooper plates containing equally laid thermocoax wires used to heat them up. By that, it is guaranteed that the temperature is homogeneously distributed on the plate. The temperature is controlled by built-in PT-1000 temperature sensors. To establish a one-dimensional temperature gradient between the two plates, a glass ring of about $10 \mathrm{~cm}$ diameter and $4 \mathrm{~cm}$ length was placed between the plates to ensure a fixed distance between them. The glass tube was helically wrapped with thermocoax wires and equipped with a further PT-1000 sensor for the temperature control as well. The glass tube ensures that the temperature of the side wall of the inner glass vessel is higher than the top part, avoiding any formation of the crystals at the wall. Three home-made temperature controllers ensured the selected temperature and thermal stability over several days or weeks. The temperature of the different components was controlled by that independently of each other. The plate sublimation method is also suitable to obtain high-quality films.

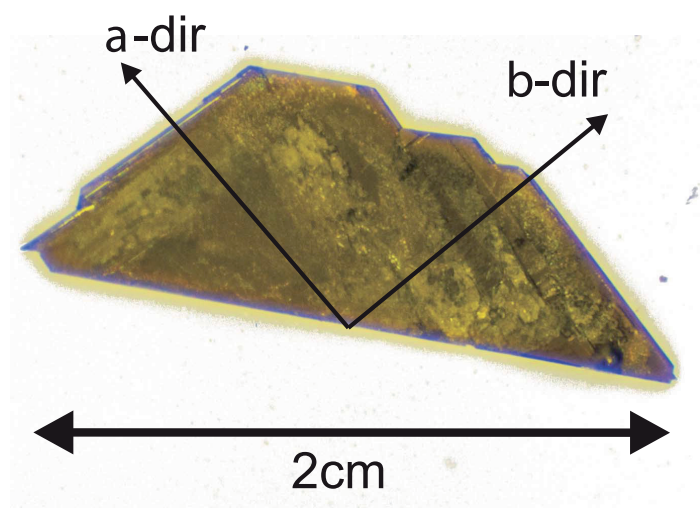

Figure 5. Photograph of a naturally grown TTF-CA single crystal which was grown by the plate sublimation method. The shape is trapezoidal with a dimension of $1 \mathrm{~mm} \times 2 \mathrm{~mm}$. The $a$ - and $b$-direction are perpendicular to the short edges of the crystal. The crystallographic axis are determined by polarized infrared measurements.

The glass box (dubbed "French cheese box") was filled with TTF and CA in a ratio of 1:1 and then evacuated. First efforts with different pressures of argon gas failed and led only to very small and thin crystals. We recognized that the growth process significantly improves when the pressure in the vessel is about $10^{-3}$ mbar without any inert gas as argon. The opening of the box was sealed by melting the glass. It was placed between the two plates in the inner part of the outer glass tube. Additionally, the whole setup was isolated by glass wool to avoid any temperature fluctuation during the crystal growth lasting for many days. Since vibrations are a crucial issue concerning the crystal growth, the whole setup was placed on heavily damped stone plates or an optical table. To evaporate and remove possible impurities on the surface of the glass vessel, the temperature was set everywhere to 
$T_{1}=T_{2}=78^{\circ} \mathrm{C}$. Afterwards, the growth process was stimulated by setting the temperature $T_{2}$ of the upper plate to $70^{\circ} \mathrm{C}$ so that the temperature difference between the two plates is $\Delta T=8^{\circ} \mathrm{C}$. Thereby, the fast growth of seed crystal is initialized. After a few hours, the temperature of the upper plate is reduced to $76^{\circ} \mathrm{C}\left(\Delta T=6^{\circ} \mathrm{C}\right)$. After two to six weeks, the crystals can be harvested by breaking the sealed glass box. Eventually, the samples are stored in an exsiccator for protection against water and kept in a refrigerator due to the high vapor pressure of TTF; the absence of bright light is advantageous. As depicted in Figure 5, the crystal glimmers greenish under light corresponding to the expected green phase of TTF-CA. Besides the TTF-CA crystals, also red and orange TTF crystals grow due to the high vapor pressure of TTF.

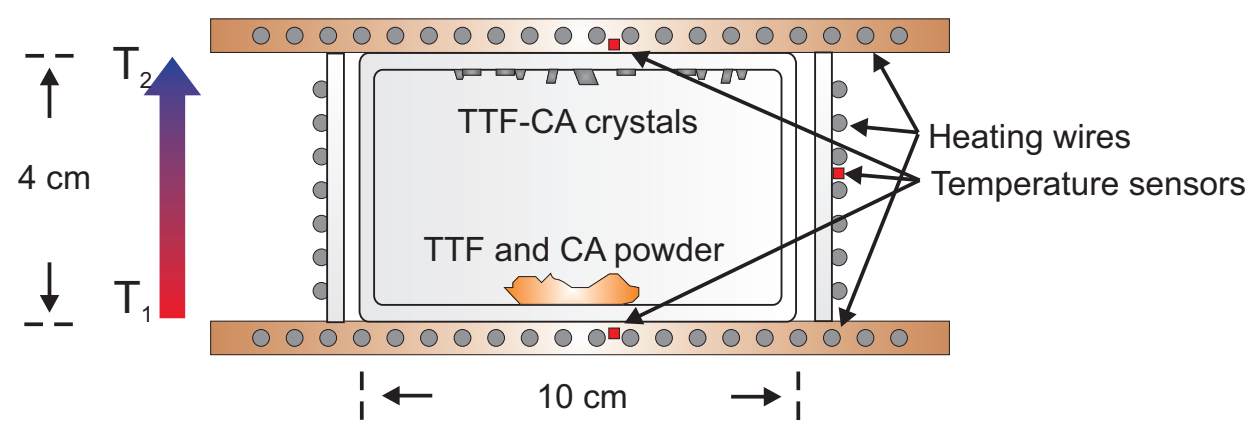

Figure 6. Schematic setup of the plate sublimation. It consists of two copper plates with incorporated meander-like thermocoax wires. A PT-1000 temperature sensor (red square) is placed in the center of each plate to check their temperature. The so-called French cheese box is located between the two plates and is surrounded by a glass ring. A third thermocoax wire is helically glued on the ring with Stycast. The temperature is controlled by a third PT-1000 in the center of the ring as well. During the growth process, a temperature gradient is set to about $\Delta T=T_{2}-T_{1}$ between the two plates. The starting powder material is placed on the bottom of the glass box. The TTF-CA crystals grow on the colder top part of the glass box.

In principle, the setup can be used for the synthesis of further kinds of charge-transfer salts, for instance TTF-TCNQ, TTF-BA. Furthermore, the method can be further optimized concerning the growth process. One idea would be to reduce the number of surface defects on the glass walls by etching or cooling down one specific point (nucleation center) on the top part of the glass vessel extremely to stimulate the growth of only a few large single crystals.

The quality of the crystals is routinely checked by electric transport measurements (see Figure 2d), optical studies (see below) and X-ray powder diffraction. In Figure 7, the room temperature diffractogram of TTF-CA crystals are compared with the one derived from the published structural data by Mayerle et al. [3]. The simulation was performed with the visualization software Diamond which is capable of generating diffraction spectra from crystallographic data. The positions of the experimental diffraction maxima (black) agree very well with the simulated values (red). The absolute intensities deviate from each other because of the imperfect milling during our sample preparation. The maximum located at $2 \Theta=28.4^{\circ}$ cannot be ascribed to TTF-CA powder but is caused by the silicon substrate material. 


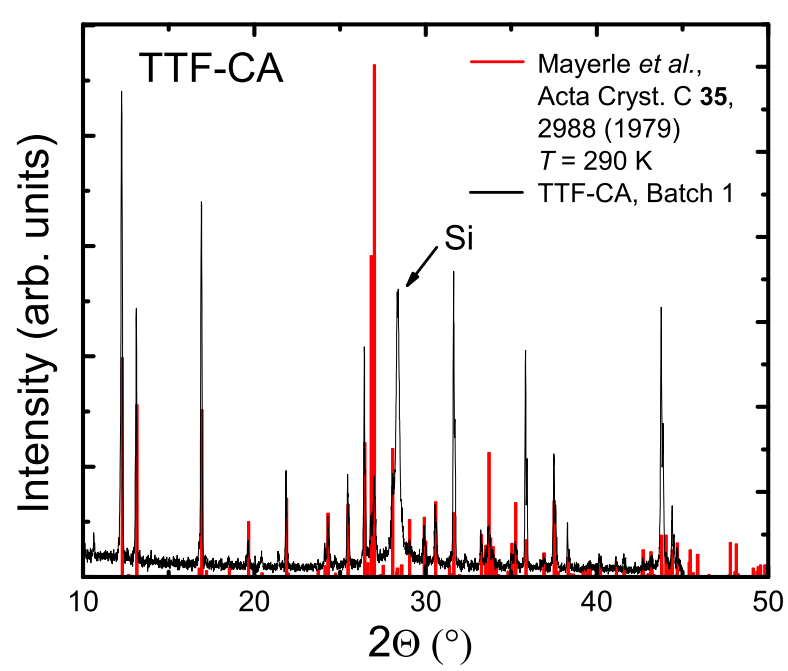

Figure 7. Comparison of the experimental powder diffractogram (black) of TTF-CA (Batch 1) with the simulated diffractogram (red) calculated from the crystal structure Mayerle et al. [3]. The broad maximum at $2 \Theta=28.4^{\circ}$ can be attributed to the silicon substrate.

\section{Ab-Initio Calculations: Band Structure, Optical Spectra and Normal Modes}

The optical spectra in the mid-infrared frequency range taken on single crystals of organic charge-transfer compounds deliver comprehensive information about the electronic, intra- and intermolecular physical properties of solid state materials. In order to understand these spectra, let us first present some theoretical considerations on the electronic band structure, the lattice and the molecular vibrations. These will be used later to interpret our results.

First, we want to concentrate on the intramolecular vibrations occurring in the TTF-CA entity. To ascribe the observed vibrational features to the corresponding molecular vibrational modes and to determine the ionicity of TTF-CA from the resonance frequency of specific modes, density-functional-theory (DFT) calculations were performed in connection with a normal mode analysis. Therefore, the $\mathrm{EDF}_{2}$-functional [42] in combination with the $6-311 \mathrm{G}^{*}(\mathrm{~d}, \mathrm{p})$ basis set was utilized. The calculations were performed with the software package Spartan [43]. The molecule structure of TTF, $\mathrm{TTF}^{+}$with the point group $\mathrm{C}_{2 \mathrm{v}}$ and CA, $\mathrm{CA}^{-}$with the symmetry $\mathrm{D}_{2 \mathrm{~h}}$ were optimized with respect to the total energy of the system in order to find the equilibrium molecular structure. The subsequent calculation did not reveal any negative or imaginary frequency, indicating that the energy minimum is reached. For the modes with a frequency above $1000 \mathrm{~cm}^{-1}$ a correction factor of 0.967 was applied whereas for the modes below $1000 \mathrm{~cm}^{-1} 1.0197$ was used [44].

In Tables $\mathrm{C} 1$ and $\mathrm{C} 2$ of Appendix $\mathrm{C}$, we give the complete list of all resonance frequencies and intensities of CA, $\mathrm{CA}^{-}$, and of $\mathrm{TTF}, \mathrm{TTF}^{+}$; the most relevant vibrational modes for our investigations are summarized in Table 2. The results agree well with previous calculations $[15,45,46]$, albeit less precise DFT-methods as basis sets were employed. For both molecules, the vibrational modes can be classified according to the $\mathrm{D}_{2 \mathrm{~h}}[47]$ symmetry as follows:

$$
\begin{gathered}
\Gamma_{\mathrm{CA}} \Rightarrow 6 a_{\mathrm{g}}+1 b_{1 \mathrm{~g}}+3 \mathrm{~b}_{2 \mathrm{~g}}+5 \mathrm{~b}_{3 \mathrm{~g}}+2 \mathrm{a}_{\mathrm{u}}+5 \mathrm{~b}_{1 \mathrm{u}}+5 \mathrm{~b}_{2 \mathrm{u}}+3 \mathrm{~b}_{3 \mathrm{u}} \\
\Gamma_{\mathrm{TTF}} \Rightarrow 7 \mathrm{a}_{\mathrm{g}}+2 \mathrm{~b}_{1 \mathrm{~g}}+3 \mathrm{~b}_{2 \mathrm{~g}}+6 \mathrm{~b}_{3 \mathrm{~g}}+3 \mathrm{a}_{\mathrm{u}}+6 \mathrm{~b}_{1 \mathrm{u}}+6 \mathrm{~b}_{2 \mathrm{u}}+3 \mathrm{~b}_{3 \mathrm{u}}
\end{gathered}
$$

Thereby, $a_{g}$, and $b_{3 u}$ from the point group $D_{2 h}$ correlate with $a_{1}$ from $C_{2 v}, a_{u}$, and $b_{3 g}$ with $a_{1}, b_{2 u}$, $b_{1 g}$ with $b_{1}$ and $b_{2 g}$ and $b_{1 u}$ with $b_{2}$. In the neutral state, the structure of TTF is bent, i.e., the C-H bonds point out of the molecule plane; similar observations are made for other common charge-transfer salts, such as TMTTF and BEDT-TTF [48]. For the calculation, we assumed therefore that the point group of TTF is $C_{2 v}$. Yet, the structure of TTF is planar in the condensed phase and allows us to imply $D_{2 h}$. The labeling of the modes in Table 2 is according to Girlando et al. [49] and Bozio et al. [47]. 
Table 2. Calculated resonance frequencies in wavenumbers, infrared intensities (Int.) and electron-molecular vibrational (emv) coupling constants $g_{i}[4]$.

\begin{tabular}{|c|c|c|c|c|c|c|c|c|c|}
\hline \multirow[b]{2}{*}{ Label } & \multirow[b]{2}{*}{ Symmetry } & \multicolumn{3}{|c|}{$\mathrm{CA}^{0}$} & \multicolumn{3}{|c|}{$\mathrm{CA}^{-}$} & \multirow[b]{2}{*}{$\begin{array}{c}\Delta v \\
\left(\mathrm{~cm}^{-1}\right)\end{array}$} & \multirow[b]{2}{*}{$\begin{array}{c}g_{i} \\
(\mathrm{meV})\end{array}$} \\
\hline & & $\begin{array}{c}v_{\text {calc }} \\
\left(\mathrm{cm}^{-1}\right)\end{array}$ & $\begin{array}{l}v_{\text {scaled }} \\
\left(\mathrm{cm}^{-1}\right)\end{array}$ & 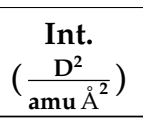 & $\begin{array}{c}v_{\text {calc }} \\
\left(\mathrm{cm}^{-1}\right)\end{array}$ & $\begin{array}{c}v_{\text {scaled }} \\
\left(\mathrm{cm}^{-1}\right)\end{array}$ & $\begin{array}{c}\text { Int. } \\
\left(\frac{\mathrm{D}^{2}}{\mathrm{amu} \AA^{2}}\right)\end{array}$ & & \\
\hline$v_{1}$ & \multirow{3}{*}{$a_{g}$} & 1754.1 & 1696.22 & - & 1548.51 & 1497.41 & - & -199 & 67 \\
\hline$v_{2}$ & & 1630.2 & 1576.24 & - & 1608.16 & 1555.09 & - & -21 & 83 \\
\hline$v_{3}$ & & 970.94 & 990.07 & - & 982.53 & 1002 & - & 12 & 95 \\
\hline$v_{10}$ & \multirow{3}{*}{$\mathrm{b}_{1 \mathrm{u}}$} & 1757 & 1699 & 349.46 & 1565.35 & 1513.7 & 288.1 & -185 & \\
\hline$v_{11}$ & & 1086.54 & 1050.68 & 415.77 & 1117.9 & 1081 & 203.72 & 30 & \\
\hline$v_{12}$ & & 899.07 & 916.78 & 25.31 & 892.53 & 910.12 & 159.74 & -7 & \\
\hline \multirow{2}{*}{ Label } & \multirow{2}{*}{ Symmetry } & \multicolumn{3}{|c|}{ TTF $^{0}$} & \multicolumn{3}{|c|}{$\mathrm{TTF}^{+}$} & \multirow{2}{*}{$\Delta v$} & \multirow{2}{*}{$g_{i}$} \\
\hline & & $v_{\text {calc }}$ & $v_{\text {scaled }}$ & Int. & $v_{\text {calc }}$ & $v_{\text {scaled }}$ & Int. & & \\
\hline$v_{2}$ & \multirow{3}{*}{$a_{g}$} & 1621.92 & 1568.39 & - & 1551.15 & 1499.96 & - & -68 & 16 \\
\hline$v_{3}$ & & 1.576 & 1524.41 & - & 1427.75 & 1380.63 & - & -144 & 115 \\
\hline$v_{4}$ & & 1.125 & 1088 & - & 1130 & 1092.71 & - & 5 & 10 \\
\hline$v_{14}$ & \multirow[b]{2}{*}{$\mathrm{b}_{1 \mathrm{u}}$} & 1598.57 & 1545.82 & 23.07 & 1532 & 1481.43 & 111.48 & -64 & \\
\hline$v_{15}$ & & 1124.65 & 1087.54 & 3.15 & 1130.92 & 1094 & 0.12 & 6 & \\
\hline
\end{tabular}

The calculations reveal an inner distance of the $\mathrm{C}=\mathrm{C}$ double bonds in $\mathrm{TTF}^{0}$ of $1.346 \AA$, which agrees very well with the experimentally determined value of $d=1.35 \AA$ in the gaseous phase [46]. In contrast, this bond length in the crystal is $d=1.3639 \AA$ [2] at room temperature. This is caused by the interaction with the neighboring molecules which deform the molecule frame. In addition, the actual charge state of TTF in the crystal at ambient conditions is not zero but finite. This is the reason why the bond length increases in comparison to the vacuum value. The calculated double bond length $\mathrm{of} \mathrm{TTF}^{+}$is increased to $d=1.395 \AA$ due to the $\pi$-orbital of the $\mathrm{C}=\mathrm{C}$ bond and corresponds very well to the behavior of TTF-CA ( $d=1.3875 \AA$ [50]) in the ionic phase at low temperatures. For CA in TTF-CA at $300 \mathrm{~K}$, the bond length of the $\mathrm{C}=\mathrm{O}$ bond is $1.214 \AA$ while the $\mathrm{C}=\mathrm{C}$ bond length is $1.352 \AA$. The calculation yields for the neutral molecule $1.205 \AA$ and $1.346 \AA$, respectively. In the ionic phase, the distance of both bonds increases to $1.2378 \AA$ and $1.366 \AA$. The theoretically derived values of $C A^{-1}$ resemble this trend excellently with $1.24 \AA$ and $1.369 \AA$.

In Table 2, the most important vibrational modes are listed with their resonance frequencies, infrared intensities and for the symmetric emv coupled modes their emv-coupling constants $g_{i}$ for the neutral as well as the charged TTF and CA molecules. Only the gerade and infrared-inactive $\mathrm{a}_{\mathrm{g}}$ and ungerade infrared-active $b_{1 u}$ modes are listed. The symmetric $a_{g}$ modes become infrared-active when they are connected to the electronic background via emv coupling (for a more detailed description see Appendix A.3). In the case of TTF-CA, this happens only in the ionic phase, since the TTF and CA molecules are dimerized along the stacking direction. The appearance of these modes in the spectrum along the $a$-direction is clear evidence of the ionic phase transition and is later used to determine the transition temperature, or in the case of the photo-induced process, to identify the transition into the neutral phase. The $v_{3}$ mode of TTF reveals the strongest coupling to the electronic background and therefore exhibits the largest modification of the intensity in the infrared spectrum.

The largest shifts of the resonance frequency as a function of ionicity are observed for the $v_{1}$ and $v_{10}$ modes of CA with a decrease of the frequency of $-199 \mathrm{~cm}^{-1}$ and $-185 \mathrm{~cm}^{-1}$, respectively. In TTF the most sensitive modes are the $v_{3}$ and $v_{14}$ which shift by $-144 \mathrm{~cm}^{-1}$ and $-64 \mathrm{~cm}^{-1}$. For determining the ionicity, the symmetric modes can be excluded since they are not visible in the neutral phase. By that, only the antisymmetric vibrations can be used for that purpose. From our calculations, it can be deduced that the $v_{10}$ mode of the CA molecule is the best choice concerning the intensity and total frequency shift because the electron density affects the bond length of the $\mathrm{C}=\mathrm{C}$ and $\mathrm{C}=\mathrm{O}$ bonds the 
most. Since this fact is included in the vibration of the $v_{10}$ mode, it is very sensitive to any modification of the molecular charge. Furthermore, the oxygen atom is deflected along the $\mathrm{C}=\mathrm{O}$ axis against the molecule body, leading to a large dipole moment. Therefore, this resonance is later used to determine the charge state of the molecules above and below the phase transition.

In the first row of Figure 8, the vibrational $\mathrm{ag}_{\mathrm{g}}$ modes of CA are displayed, being symmetric to symmetry operations of the point group $\mathrm{D}_{2 \mathrm{~h}}$, together with the antisymmetric, infrared-active $\mathrm{b}_{1 u}$ mode. Similar to that, the most important modes of the TTF molecules are depicted in Figure 9. Again, the electron density is highest on the $\mathrm{C}=\mathrm{C}$ double bond, which has the largest impact on any modification of the potential energy surface. Although vibrations of the outer methyl groups take place, they have only little influence on the dipole moment.
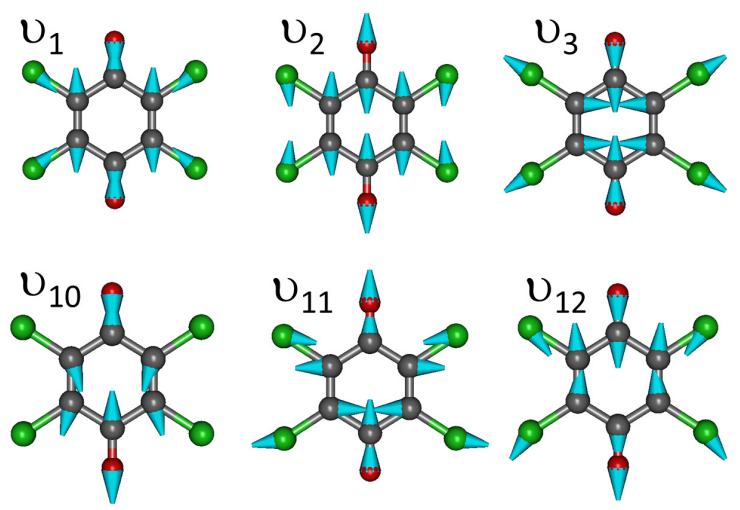

Figure 8. In the upper row, the symmetric ag-modes $v_{1}, v_{2}$, and $v_{3}$ of CA are visualized. The ungerade $\mathrm{b}_{1 u}$-modes $v_{10}, v_{11}$, and $v_{12}$ are listed in the lower row.

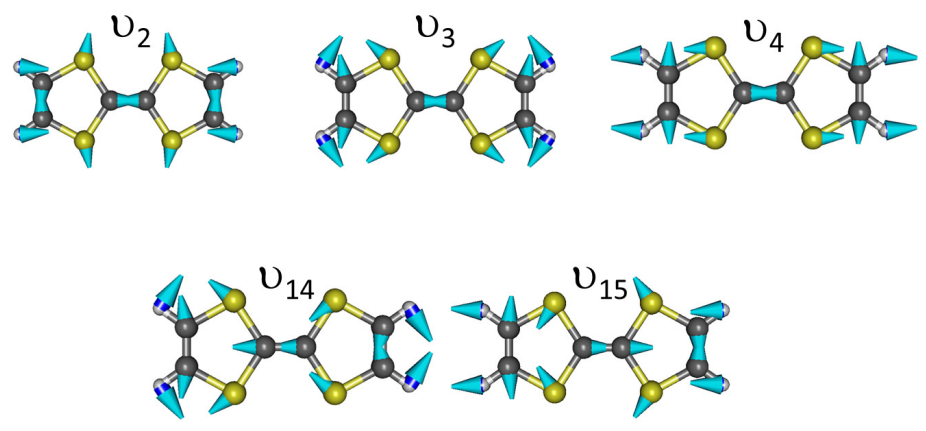

Figure 9. In the upper row, the gerade $\mathrm{a}_{\mathrm{g}}$ modes $v_{2}, v_{3}$, and $v_{4}$ of TTF are depicted. The ungerade $\mathrm{b}_{1 u}$ mode $v_{14}$ and $v_{15}$ are displayed in the second row.

For the band structure calculations, we used the DFT method in connection with pseudopotentials and plane waves, implemented in the software suite Quantum Espresso [51]. In the framework of this theoretical consideration, the generalized gradient approximation (GGA) Perdew-Burke-Enzerhof (PBE) functional [52] together with ultrasoft pseudo potentials [53] for all atomic types is employed. The cutoff-energy of the wave function was set to $E_{\text {Wave }}=30 \mathrm{eV}$. The selected electron density cutoff-energy was fixed to $E_{\rho}=450 \mathrm{eV}$. For the self-consistent calculation, an equally-spaced Monkhorst-grid $(6 \times 6 \times 4)$ was generated [54]. The band structure was determined along the $k$-path: $\Gamma(0,0,0) \rightarrow Z(0,0,0.5) \rightarrow \Gamma(0,0,0) \rightarrow Y(0,0.5,0) \rightarrow U(0,0.5,0.5) \rightarrow \Gamma(0,0,0) \rightarrow X(0.5,0,0) \rightarrow$ $S(0.5,0.5,0) \rightarrow \Gamma(0,0,0)$. In Figure 10, the band structure in an energy range from $-2 \mathrm{eV}$ to $3 \mathrm{eV}$ is depicted for the ionic and neutral phase of TTF-CA. Between $-0.5 \mathrm{eV}$ and $0.5 \mathrm{eV}$, the valence and conduction bands are located, which are separated from each other by a band gap of $0.02 \mathrm{eV}$ at $300 \mathrm{~K}$ and $0.23 \mathrm{eV}$ at $15 \mathrm{~K}$. Each of the single bands are split into two bands due to the slightly interacting 
neighboring TTF-CA-chains within the unit cell. The highest occupied molecular orbital (HOMO) of the TTF molecule contributes to the two lower bands whereas the upper bands are dominated by the lowest unoccupied molecular orbital (LUMO) of the CA molecule.
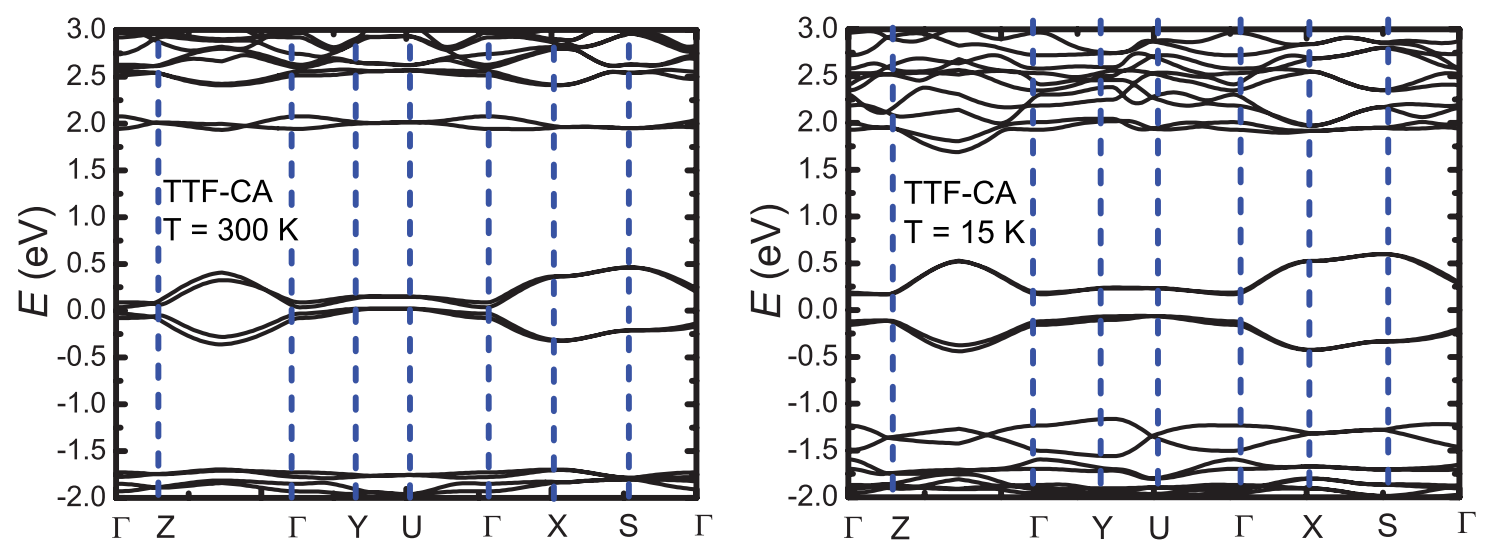

Figure 10. Band structure of TTF-CA at room temperature $(T=300 \mathrm{~K})$ and low temperatures $(T=15 \mathrm{~K})$ along the selected $k$-path in an energy range from $-2 \mathrm{eV}$ to $3 \mathrm{eV}: \Gamma(0,0,0) \rightarrow Z(0,0,0.5) \rightarrow \Gamma(0,0,0) \rightarrow$ $Y(0,0.5,0) \rightarrow U(0,0.5,0.5) \rightarrow \Gamma(0,0,0) \rightarrow X(0.5,0,0) \rightarrow S(0.5,0.5,0) \rightarrow \Gamma(0,0,0)$. The band gaps are $0.02 \mathrm{eV}$ at $\mathrm{T}=300 \mathrm{~K}$ and $0.23 \mathrm{eV}$ at $15 \mathrm{~K}$.

The energy dispersion along $\Gamma \rightarrow X$ is obviously maximal along the stacking direction (a-axis). The band width $W$ of the conduction band is about $W=4 t=410 \mathrm{meV}$ at $300 \mathrm{~K}$ and temperature-independent. The valence band reveals a band width of $W=4 t=300 \mathrm{meV}$ and increases to $360 \mathrm{meV}$ upon cooling. Along the other directions, the dispersion is very weak; it implies that TTF-CA is a one-dimensional system. The optical measurements (Figure 11) show that an extensive maximum is located at about $0.6 \mathrm{eV}$ (corresponding to $4800 \mathrm{~cm}^{-1}$ ), which is caused by the excitations from the valence to the conduction band in the regions where the energy difference of the two states $\Delta=E_{\mathrm{CB}}-E_{\mathrm{VB}}$ is almost constant over a wide range, meaning that the deviation in the denominator $\nabla_{k} \Delta E$ tends to zero [55]. For these so-called critical points, the joint density of states, defined as $D\left(E_{\mathrm{VB}}\right) \propto \int \frac{d S_{k}}{\nabla_{k} \Delta E}$, becomes extremely large in this energy range and area in the $k$-space. It corresponds to a maximum in the optical conductivity. The valence and conduction bands are well separated from the other bands; hence TTF-CA can be considered as a two band system by neglecting the lifting of the degeneracy of the valence and conduction bands. The energy difference to the lower bands is about $1.3 \mathrm{eV}$ and decreases to $0.7 \mathrm{eV}$ at low temperatures. This is similar to the upper band whose distance reduces from $1.5 \mathrm{eV}$ at $300 \mathrm{~K}$ to $1.1 \mathrm{eV}$ at $15 \mathrm{~K}$. Therefore, it is not surprising that in the visible range strong modifications of the optical properties of TTF-CA occur when going from the neutral to the ionic phase.

In addition to the band structure, we theoretically determined the optical conductivity according to Equation (B9) for all crystallographic directions by calculating the electronic transition of 200 equally spaced $k$-points in the Brillouin zone. The findings for three different temperatures are presented in Figure 12: $T=300 \mathrm{~K}, 105 \mathrm{~K}$ and $15 \mathrm{~K}$. Here, we used norm-conserving PBE pseudo potentials at which the band gap was determined to $0.11 \mathrm{eV}$ at $300 \mathrm{~K}$ and $0.28 \mathrm{eV}$ at $15 \mathrm{~K}$. They agree very well with previous calculations [56] based on local density approximation (LDA) but their results are closer to the experimentally determined gap of $0.6 \mathrm{eV}$. It is well known, however, that especially the LDA functional underestimates the experimental band gap more than the GGA functional and as PBE. 


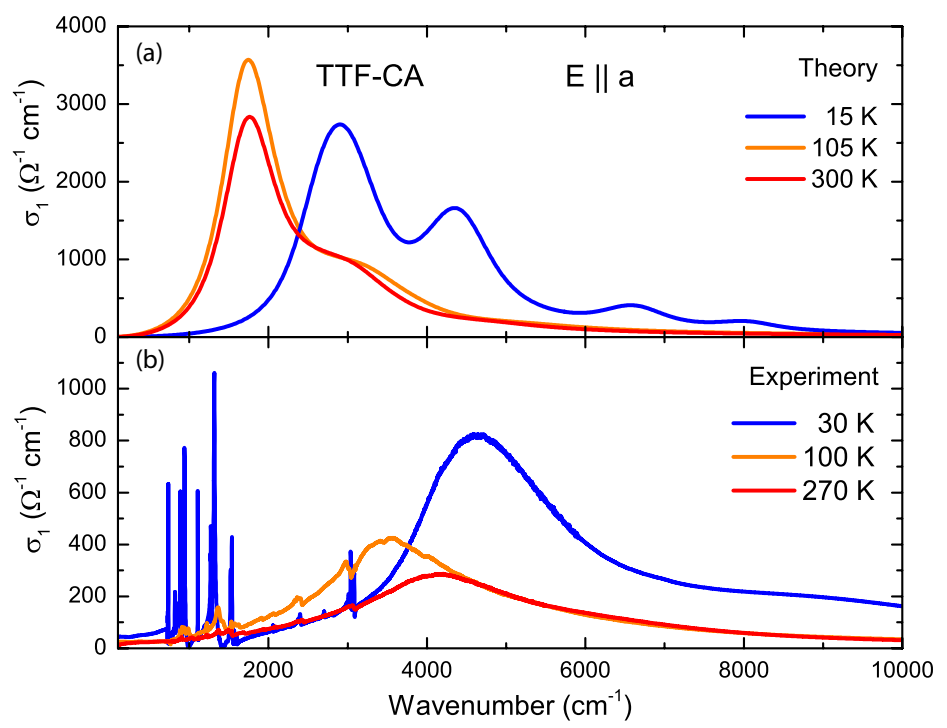

Figure 11. (a) Optical conductivity $\sigma_{1}(v)$ of TTF-CA at $T=15 \mathrm{~K}$ (blue), $105 \mathrm{~K}$ (orange) and $300 \mathrm{~K}$ (red) along the stacking direction. The calculations were performed with the method described in Appendix B. Various electronic transitions between the lower valence bands and the unoccupied conduction band are visible; (b) Experimentally determined optical conductivity $\sigma_{1}(v)$ of TTF-CA at $T=30 \mathrm{~K}, 100 \mathrm{~K}$, and $270 \mathrm{~K}$. In the range between $3000 \mathrm{~cm}^{-1}$ and $10,000 \mathrm{~cm}^{-1}$ the electronic excitations are located while the sharp features below $3000 \mathrm{~cm}^{-1}$ can be ascribed to intramolecular vibrations.
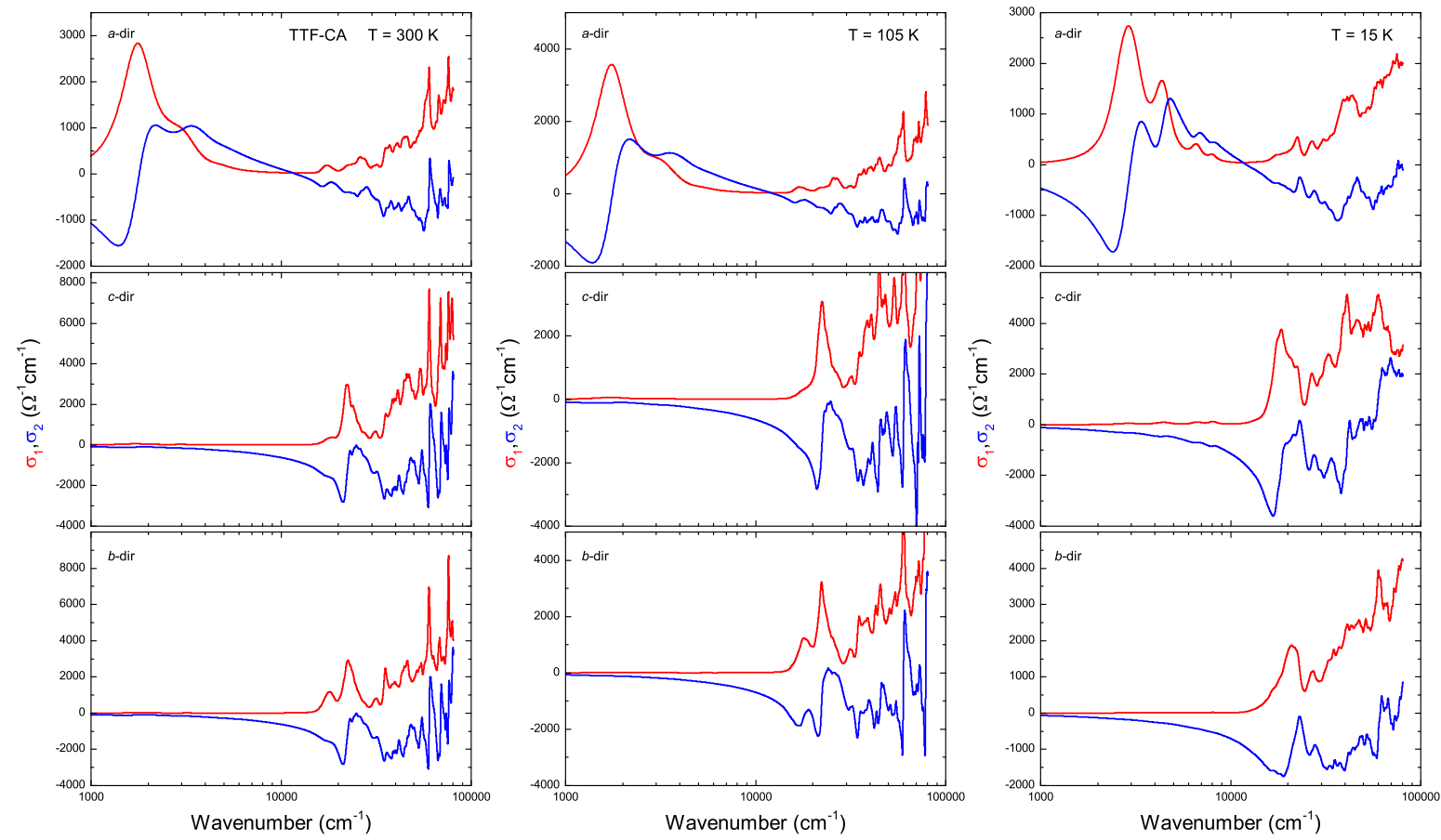

Figure 12. Real and imaginary parts of the frequency dependent conductivity $\sigma_{1}(v)$ (red lines) and $\sigma_{2}(v)$ (blue lines) of TTF-CA calculated by density functional theory described in Appendix B for all three crystallographic axes. The left column displays the optical conductivity at ambient conditions, the central column at $T=105 \mathrm{~K}$ and the right column at low temperatures $(T=15 \mathrm{~K})$. For the calculation, the structural data of TTF-CA were used, taken at the corresponding temperatures $[2,50]$.

In Figure 11, the calculated optical conductivity $\sigma_{1}(v)$ is compared to the experimentally determined conductivity measured in a frequency range from $500 \mathrm{~cm}^{-1}$ to $10,000 \mathrm{~cm}^{-1}$ for $E \| a$. 
For a better comparison, the transitions were folded with a Lorentz function of $800 \mathrm{~cm}^{-1}(0.1 \mathrm{eV})$ width. The maximum in Figure 11a is located at $v=1700 \mathrm{~cm}^{-1}(0.22 \mathrm{eV})$ at room temperature and does not shift with temperature till $100 \mathrm{~K}$. Below the neutral-ionic phase transition, the maximum is suddenly located at higher energies at $2900 \mathrm{~cm}^{-1}(0.36 \mathrm{eV})$ due to the increase of the band gap in the ionic phase. In addition, other conductivity maxima show up, which originate from transitions between the valence and conduction bands with a large joint density of states due to a constant $\Delta E$ between the bands; for instance at the symmetry points $X$ or $S$. The temperature-dependent shift of the spectra agrees qualitatively very well with the optical study. Only the absolute positions of the resonance frequencies of the experiments are located at higher energies, between $1400 \mathrm{~cm}^{-1}$ and $2400 \mathrm{~cm}^{-1}$, which can be attributed to the underestimation of the gap by the DFT method. Furthermore, the experimental mid-infrared interband transition is most likely composed of several excitations, which are rendered by the simulation. They are added up to a single broad feature. Supporting evidence comes from the long tail above $6000 \mathrm{~cm}^{-1}$ in the case of the $T=30 \mathrm{~K}$ spectra indicating the fact that more electronic excitations are involved in this optical band. This probably explains the wavelength-dependent generation efficiency in photo-induced phase transition in TTF-CA as up to now less is discussed about the composition of this band. Above the phase transition, $T>T_{\mathrm{NI}}$, a slight red shift of the interband transition by about $600 \mathrm{~cm}^{-1}$ is observed as the temperature is reduced from room temperature down to $100 \mathrm{~K}$; this phenomenon is explained by thermal contraction of the crystal similar to hydrostatic pressure. The sharp features below $v=3000 \mathrm{~cm}^{-1}$ can be related to intramolecular modes of the TTF and CA molecules and do not influence the electronic excitations. In the experimental spectra, no additional features could be detected in the examined spectral range, indicating the excitation of solitons or NIDW.

\section{Infrared Measurements}

We have performed comprehensive optical investigations of TTF-CA single crystals along the crystallographic $a$ - and $b$-axes, using standard Fourier-transform infrared spectroscopy $[57,58]$. In a first step, we measure the temperature-dependent reflectivity and derive the steady-state properties in order to compare them later with photo-induced measurements and learn something about the dynamics at the neutral ionic phase transition. As mentioned in the previous Section 3, the spectra along the stacking directions contain information about the molecular vibrations of the single molecules within the unit cell as well as about electronic excitations. In contrast, no inter- and intraband transitions occur along the $b$-direction allowing a clear view on the infrared-active molecular vibrational modes. The following paragraph is dedicated to the optical properties of the $a$ - and $b$-axes and their temperature-dependent behavior.

\section{1. b-Direction}

Since there are no electronic excitations present in the spectra with light polarized parallel to the $b$-axis, the odd modes of TTF and CA can be observed without any problems. The $b_{1 \mathrm{u}}$-modes appear mainly for $E \| b$, since their dipole moments are oriented along the long molecular axis (compare therefore Figures 8 and 9). However, because of the arrangement of the molecules in the unit cell, also vibrational modes with the symmetry $b_{2 u}$ can be found whose dipole moments are within the molecular plane, but perpendicular to the long molecular axis.

In Figure 13, details of the reflectivity $R(v)$ and optical conductivity $\sigma_{1}(v)$ are displayed for four different temperatures measured on a TTF-CA single crystal with $E \| b$. The Kramers-Kronig transformation was performed by extrapolating the low-frequency range with a constant value. For the extrapolation above $6000 \mathrm{~cm}^{-1}$ up to $500,000 \mathrm{~cm}^{-1}$ a $v^{-4}$-decay was applied. The average reflectivity for the $b$-axis is $10 \%$. Between $v=750 \mathrm{~cm}^{-1}$ and $920 \mathrm{~cm}^{-1}$ two modes are located. The energetically lower resonance at about $800 \mathrm{~cm}^{-1}$ can be assigned to the $v_{16}$-mode of TTF which blue-shifts on cooling and undergoes a sudden jump of $10 \mathrm{~cm}^{-1}$ at the phase transition. The intensity also increases slightly, as predicted by the theoretical calculations (see Table C2 in Appendix C). At room temperature, further 
weak maxima can be recognized at higher frequencies, which we ascribe to the $v_{25}\left(b_{2 u}\right)$-mode of TTF [47]. According to Table C2, theory predicts the mode to appear at $v=802 \mathrm{~cm}^{-1}$, but it is shifted due to the ionicity, in this case to $805 \mathrm{~cm}^{-1}$. In the ionic phase, a weak satellite peak appears above the $v_{16}$-mode at about $820 \mathrm{~cm}^{-1}$ as well (not shown). The $v_{12}$-mode of CA located at about $905 \mathrm{~cm}^{-1}$ shifts a bit to higher energies and gains intensity. The calculations predict a minimal increase of a factor of 6 . In summary, for both considered modes the total shift is moderate.

In Figure 13c,d, the frequency range between $1050 \mathrm{~cm}^{-1}$ and $1550 \mathrm{~cm}^{-1}$ is visualized. The frequency band at $1100 \mathrm{~cm}^{-1}$ is dominated by the $v_{11}$-mode of CA accompanied by the $v_{15}$-mode of TTF. Both modes experience a positive shift of a few wavenumbers on cooling. In particular, the $v_{11}$-vibration hardens appreciably. In the range of $1500 \mathrm{~cm}^{-1}$ only one feature is pronounced at $v=1540 \mathrm{~cm}^{-1}$ in the neutral phase that is related to the $v_{14}$-mode of TTF. In the ionic phase, it is located at $1520 \mathrm{~cm}^{-1}$. In addition, another mode appears at $1510 \mathrm{~cm}^{-1}$ and $1500 \mathrm{~cm}^{-1}$, which can be ascribed to the symmetric $v_{2}$-vibration of TTF that becomes infrared-active due to emv-coupling in the modified crystal field below the phase transition. However, it can also be assigned to a combination or higher harmonic mode, which becomes possible due to the altered potential energy surface of the molecules.

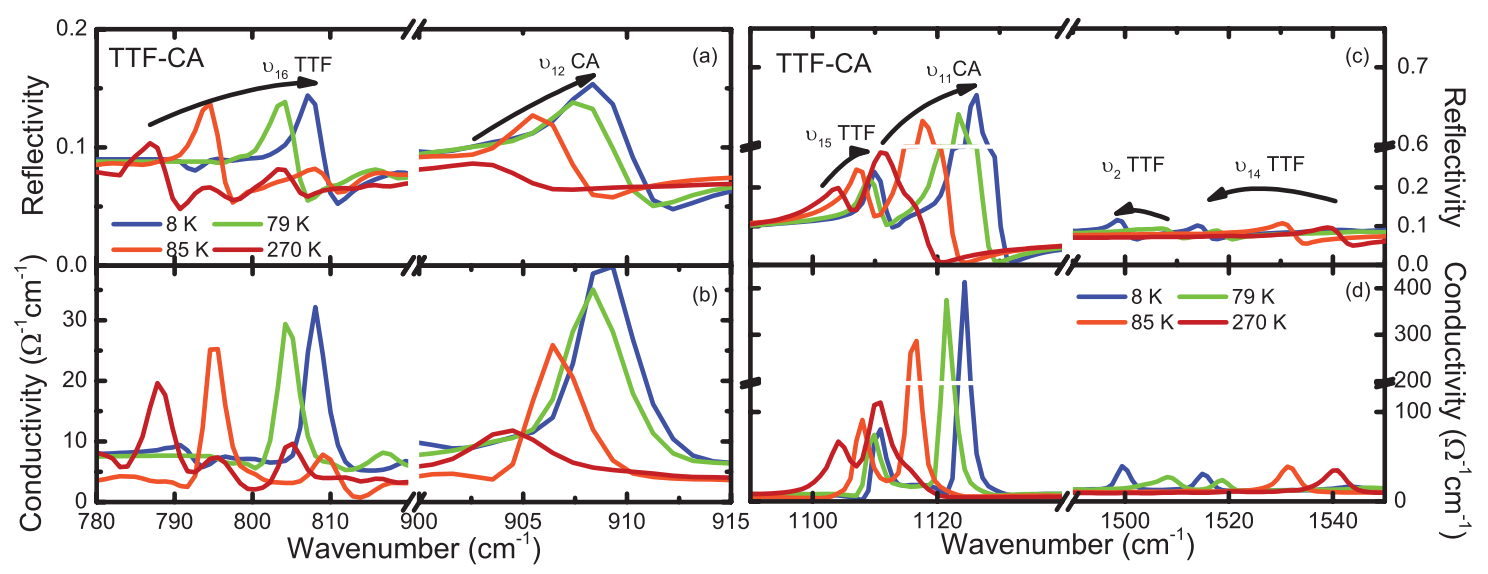

Figure 13. (a,c) Reflectivity and (b,d) optical conductivity of TTF-CA as a function of temperature in the two different frequency ranges taken at different temperatures as indicated. $(\mathbf{a}, \mathbf{b})$ The first mode in the region from $780 \mathrm{~cm}^{-1}$ to $920 \mathrm{~cm}^{-1}$ can be assigned to the $b_{1 \mathrm{u}} v_{14}$ of TTF. The neighboring maximum belongs to the antisymmetric $v_{12}$-mode of CA, as listed in Table 2; (c,d) Between $1000 \mathrm{~cm}^{-1}$ and $1550 \mathrm{~cm}^{-1}$ four modes appear whose resonance frequency shifts with decreasing temperature. The $v_{11}$ mode of CA at $v=1120 \mathrm{~cm}^{-1}$ has the strongest intensity while the antisymmetric modes of TTF are barely noticeable.

In order to closer inspect the $v_{10}$ and $v_{2}$ vibrational modes of CA in Figure 14, the spectral ranges around $1600 \mathrm{~cm}^{-1}$ is plotted. The $v_{2}$ feature appears only in the ionic phase and does not exhibit any temperature dependence; therefore, we suppose a symmetric mode becoming infrared-active due to the dimerization below $T_{\mathrm{NI}}$. In contrast, the antisymmetric $v_{10}$ vibration reveals a strong shift with decreasing temperature and hence, a change of the molecular ionicity. Furthermore, the intensity increases strongly below the phase transition. Hence, this mode is definitely the first choice to determine the charge state of the molecule within the different phases. For the antisymmetric Raman modes, it was shown previously that a linear relation between the molecular charge and the resonance frequency holds. Girlando et al. [59] have calculated that for the symmetric $\mathrm{ag}_{\mathrm{g}}$-mode, the resonance frequency changes nonlinearly with charge per molecules; thus these modes are not well suited to determine the ionicity. 

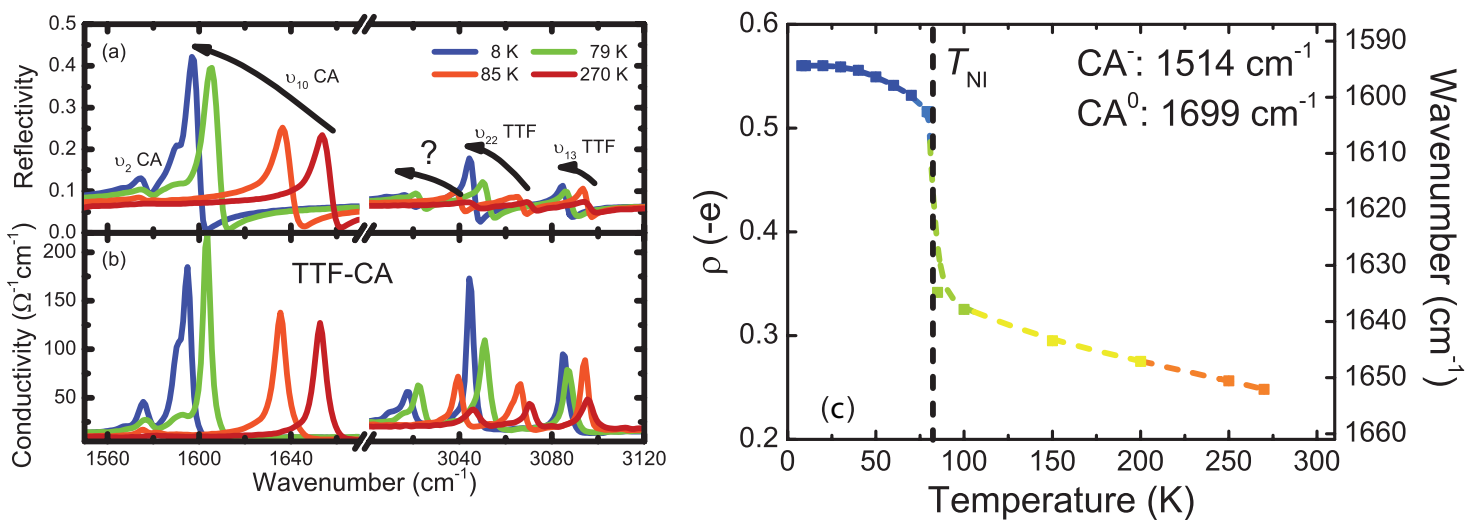

Figure 14. (a) Reflectivity and (b) optical conductivity of TTF-CA for four different temperatures between $1550 \mathrm{~cm}^{-1}$ and $1670 \mathrm{~cm}^{-1}$. The first mode is ascribed to the charge sensitive, ungerade $\mathrm{b}_{1 \mathrm{u}} v_{10}$ mode of CA. The neighboring satellite peak belongs to the symmetric $v_{2}$-mode of CA; (c) Temperature-dependent evolution of the resonance frequency of the $v_{10}$-mode. From Equation (4) and the calculated values in Table $\mathrm{C} 1$, the ionicity of CA can be determined. At the phase transition, the molecular charge jumps from $0.34 e$ to $0.51 e$ and increases moderately.

With the help of the resonance frequency of the $v_{10}$ mode of CA and the calculated values for $v_{10}$ from Table $\mathrm{C} 1$ in Appendix $\mathrm{C}$ and the equation

$$
\rho_{\mathrm{t}}=\frac{\nu_{0}-1699 \mathrm{~cm}^{-1}}{185 \mathrm{~cm}^{-1} / e},
$$

the molecular charge $\rho_{t}$ can be derived at which the resonance frequency of a neutral CA molecule is $1699 \mathrm{~cm}^{-1}$, while the total difference between the fully charged and neutral CA molecule is $-185 \mathrm{~cm}^{-1}$. In the neutral phase, the electronic charge changes from $0.25 e$ to $0.35 e$ at $T=85 \mathrm{~K}$ on cooling; i.e., the charge transfer increases slightly with decreasing temperature. Below $85 \mathrm{~K}$ at $T_{\mathrm{NI}}=81.5 \mathrm{~K}$, the charge is suddenly redistributed so that $\rho_{t}$ jumps to $0.52 \mathrm{e}$. This abrupt change is a direct hallmark of a first-order transition. Another typical behavior is the appearance of hysteresis between the cooling and heating cycle, which indeed we have observed in our optical measurement by a very slow cooling rate (not presented here). The difference between the coercive temperatures is $3 \mathrm{~K}$. This observation agrees very well with previous measurement of the heat capacity and the crystal structure [60].

The methyl vibrations of the TTF molecule are located mainly in the range around $3000 \mathrm{~cm}^{-1}$, as illustrated in the right frame of Figure 14a,b. There, several resonances can be identified, which are connected to different vibrational states of $\mathrm{CH}_{3}$. Two of the three modes can be assigned-in accordance to the values in Table $\mathrm{C} 2-$ to $v_{22}\left(\mathrm{~b}_{2 \mathrm{u}}\right)$ and $v_{13}\left(\mathrm{~b}_{1 \mathrm{u}}\right)$-modes. The third maximum cannot be ascribed to a specific vibration; it may be the emv-coupled $\mathrm{ag}_{\mathrm{g}}$ or the second harmonic of the $v_{14}$-mode.

To analyze the spectra quantitatively, we fitted resonances in the optical conductivity $\sigma_{1}(v)$ as well as the reflectivity $R(v)$ simultaneously with Fano functions (for more details we refer to Appendix A.3). The temperature-dependent evolution of all resonance frequencies is depicted in Figure 15. Without exception, all modes reveal a slight shift within the neutral phase, but at $T_{\mathrm{NI}}$ their frequencies change suddenly marking the phase transition. Below the transition, it saturates at a fixed value below $20 \mathrm{~K}$. This is originally caused by the thermal contraction of the unit cell enhancing the charge transfer. It is interesting to note that this behavior is in strong contrast to the behavior of the TMTTF-salts [48] where the transition of the charge ordered state evolves continuously indicated by the charge imbalance. In this way, it resembles the behavior observed at the charge-order phase transition of $\alpha$-(BEDT-TTF $)_{2} \mathrm{I}_{3}$ where a strong involvement of the lattice was shown recently [61-64]. Moreover, several features reveal a positive shift instead of a negative one. The sign of the frequency shift depends on the intermolecular forces and bond lengths which are a function of the charge distribution within the molecule, therefore they depend on the charge state of the molecule. In principle, every mode can be 
used to determine the ionicity. However, the analysis of the $v_{10}$ mode of CA has significant advantages compared to other modes: The total shift between the neutral and positive charged molecules is with $-185 \mathrm{~cm}^{-1} / e$ extremely large. Also, the infrared intensity is the strongest, corresponding to the calculation. Furthermore, in good approximation, the relation between charge and frequency is linear.
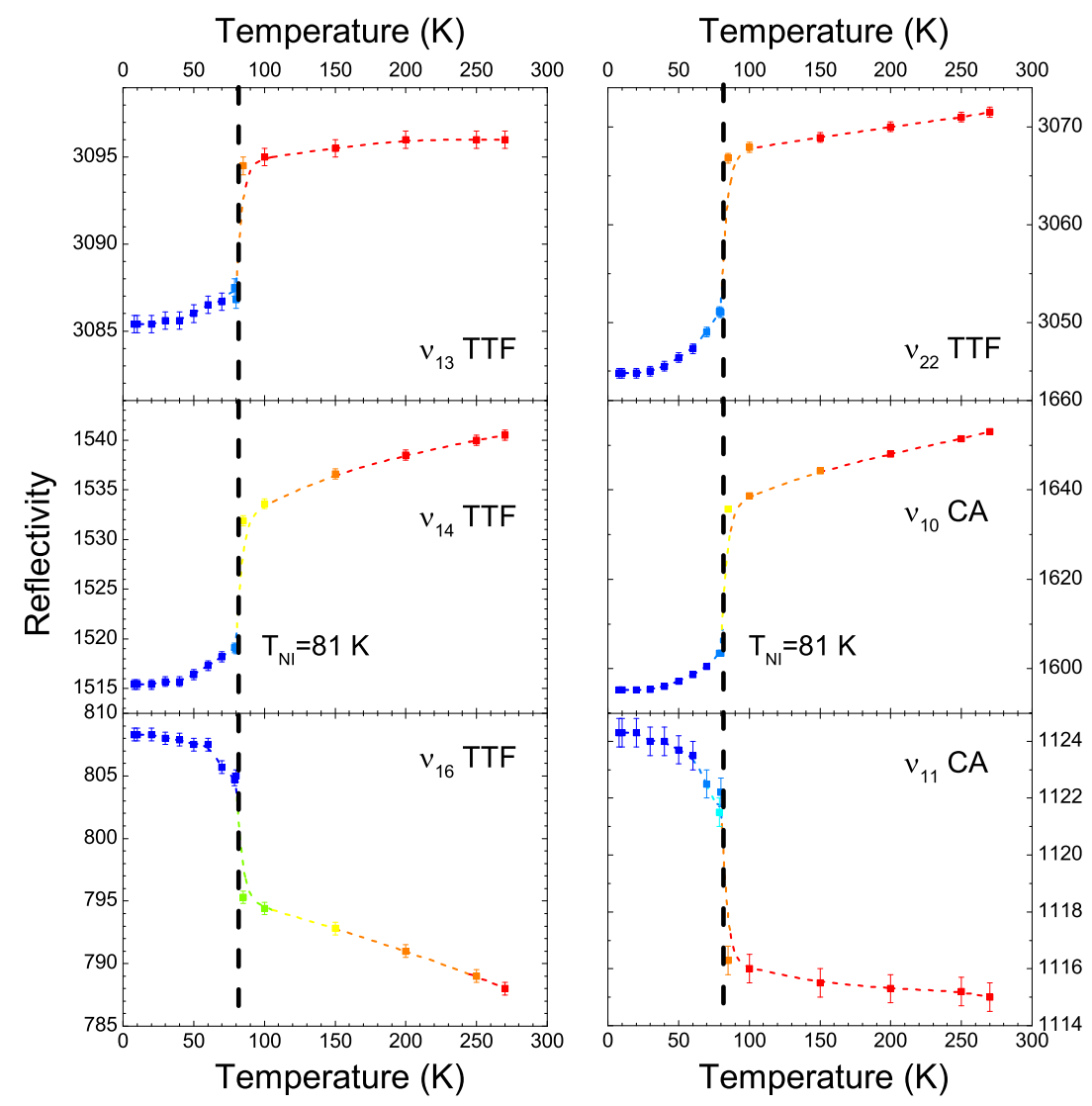

Figure 15. Temperature dependence of different vibrational frequencies observed from reflection measurements of TTF-CA with light polarized along the $b$-direction. In all cases, a pronounced shift-although in different directions-is observed at the neutral-ionic phase transition, with indications of fluctuations above and below $T_{\mathrm{NI}}$.

\section{2. a-Direction}

In addition, we measured the optical spectrum for $E \| a$ for various temperatures above and below the phase transition $T_{\mathrm{NI}}$. Since TTF-CA is insulating in the neutral phase, one would in general extrapolate the low-frequency part with a constant value. However, we know that below $200 \mathrm{~cm}^{-1}$ very strong lattice vibrations exist [65], which have a strong impact on the reflectivity and lift it up to almost $R=0.6$. Therefore, an effective reflectivity function was used as an extrapolation in this frequency range resembling exactly this behavior. It consists of an averaged spectrum of the far-infrared measurement [65]. For the high frequency part, a $v^{-4}$ extrapolation was employed up to $500,000 \mathrm{~cm}^{-1}$.

In Figure 16, the reflectivity and the optical conductivity of TTF-CA along the stacking direction is exemplary plotted for a few temperatures between $700 \mathrm{~cm}^{-1}$ and $7000 \mathrm{~cm}^{-1}$. The most prominent feature is the charge-transfer band whose maximum at ambient temperature is located at $4000 \mathrm{~cm}^{-1}$ and shifts about $500 \mathrm{~cm}^{-1}$ to $3500 \mathrm{~cm}^{-1}$ at $85 \mathrm{~K}$ on cooling. After the transition, the maximum jumps to $v \approx 4500 \mathrm{~cm}^{-1}$ due to the hybridization of the TTF and CA orbitals in the ionic phase and therewith the related charge transfer, by which the ionicity is also increased. For further cooling, the maximum moves are barely observable at about $100 \mathrm{~cm}^{-1}$ to $4600 \mathrm{~cm}^{-1}$. Additionally, the intensity 
rises drastically. The absolute values agree very well with previous measurements [5]. As shown above in Section 3, the broad band consists of several narrower features expressed in the experimental spectrum as a shoulder connected to the maximum at higher frequencies. We attribute this to the excitation of an interdimer transition [5]. Similar observations were made earlier in the charge-transfer salt K-TCNQ [66] and related to the excitations into the conduction band. This interpretation is conform with our calculation that it is a transition from the HOMO (valence band) to the LUMO (conduction band).

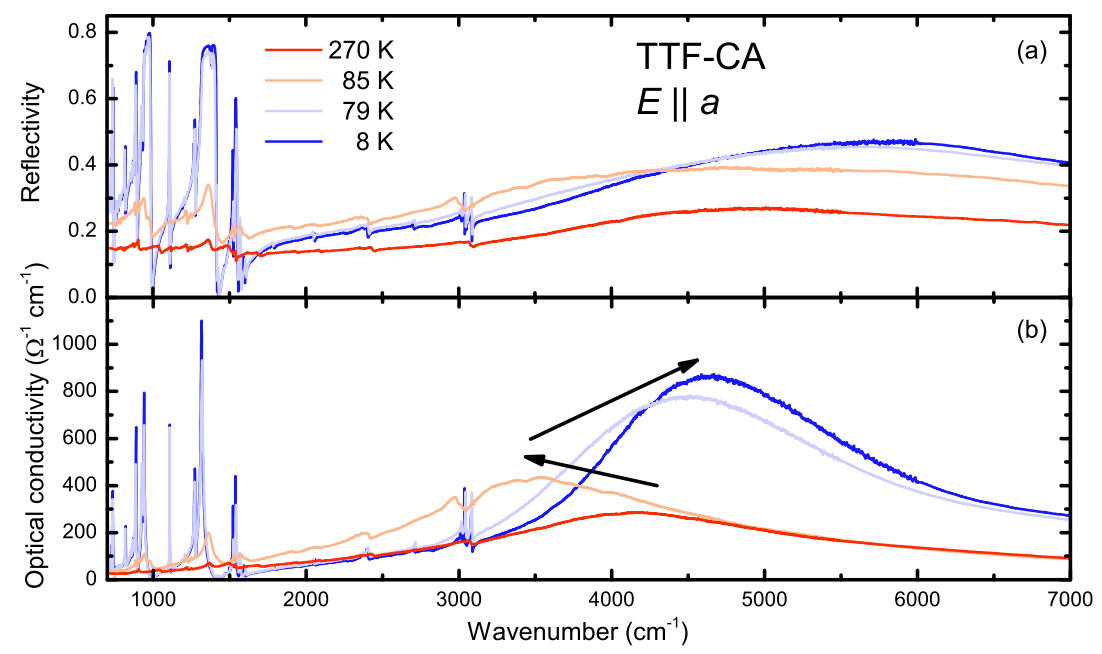

Figure 16. (a) Reflectivity and (b) optical conductivity of TTF-CA measured in the mid-infrared range for $E \| a$ from $T=295 \mathrm{~K}$ to $8 \mathrm{~K}$. The spectrum is divided into the region from $700 \mathrm{~cm}^{-1}$ to $3200 \mathrm{~cm}^{-1}$ where intramolecular modes dominate, and in the higher frequency range, where the features originate from electronic excitations.

To make a precise statement, the spectra were fitted for the electronic excitation with a Lorentz function and the molecular vibrations with Fano functions (see Appendices A.2 and A.3). From the mid-infrared band, the transfer integral $t$ and the charge transfer $\rho_{t}$ can be determined by the following relation [67] starting with the oscillator strength $f_{\text {osc: }}$ :

$$
f_{\mathrm{osc}}=\frac{\omega_{p}^{2} m \epsilon_{0}}{4 \pi N e^{2}}
$$

with $N$ the number of dimer pairs in the unit cell and $\omega_{p}$ the plasma frequency of the resonance. The charge transfer $\rho_{t}$ is related to the transfer integral $t$ via the charge-transfer band $\hbar \omega_{\mathrm{CT}}$ according to

$$
\rho_{\mathrm{t}}=\frac{3 e t^{2}}{2\left(\hbar \omega_{\mathrm{CT}}\right)^{2}} \text {. }
$$

By using the charge amount of $\rho_{t}$ determined from the $v_{10}$ mode by our optical experiments along the $b$-axis (see Section 4.1 above) and the resonance frequency $\omega_{\mathrm{CT}}$ of the mid-infrared band, depicted in Figure 17, the transfer integral $t$ can be deduced. We suppose that, on cooling, $t$ only varies slightly since $\rho_{t}$ rises and $\omega_{\mathrm{CT}}$ decreases in the neutral phase before both quantities jump abruptly at the phase transition accordingly to the behavior of $\rho_{t}$ and $\omega_{\mathrm{CT}}$. From the integral $t$, the bandwidth $W=4 t$ can be estimated with values of about $0.8 \mathrm{eV}$ in the neutral phase and $1.3 \mathrm{eV}$ at $T=8 \mathrm{~K}$. If we now compare these numbers to the total bandwidth of the calculated bands in Section 3, which is $0.7 \mathrm{eV}$ at room temperature and $1 \mathrm{eV}$ at $T=15 \mathrm{~K}$, they match nicely and reproduce the properties of the material accurately. The temperature-dependent behavior is illustrated in Figure 17b together with the oscillator strength $f_{\text {osc }}$. The latter quantity reflects very well the trend of the mid-infrared band 
intensity: $f_{\text {osc }}$ gains drastically on strength from 0.1 to 0.6 and thus dominates the optical response in this frequency range.
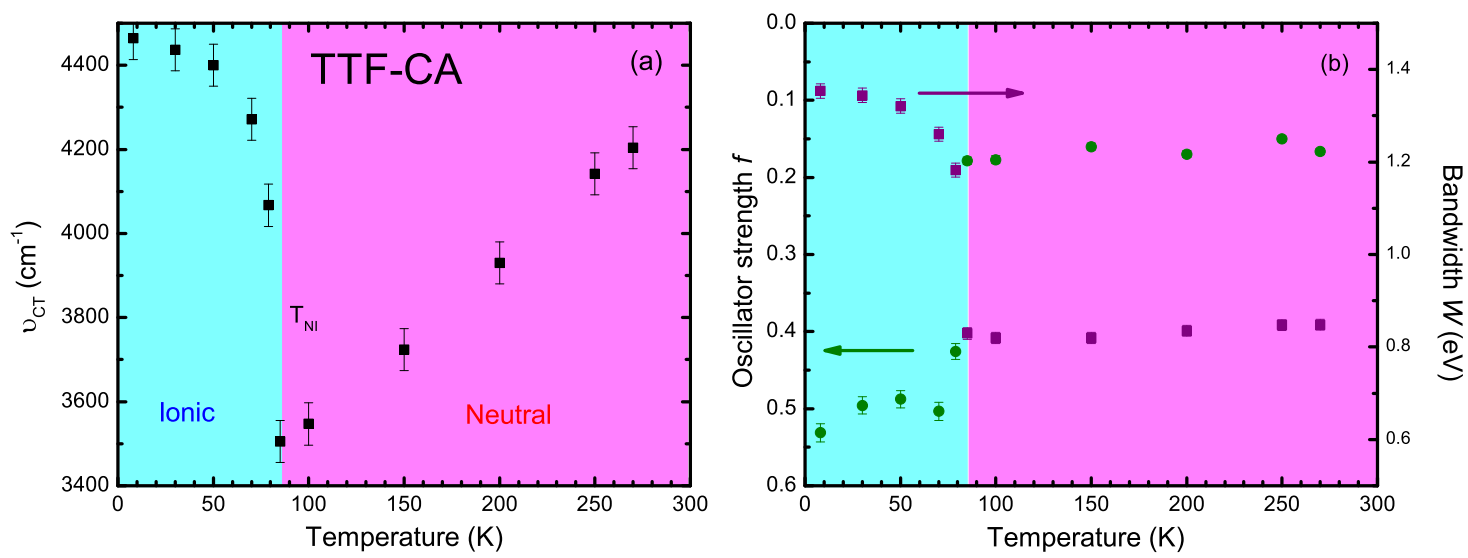

Figure 17. (a) Resonance frequency of the charge-transfer band of TTF-CA as a function of temperature; (b) Temperature dependence of the oscillator strength of the charge-transfer band (left scale) and the bandwidth for different temperatures (right scale).

In Figure 18, the mid-infrared reflectivity and the optical conductivity of TTF-CA are plotted for temperatures above and below $T_{\mathrm{NI}}$. In this energy range, the intramolecular modes of the TTF and CA molecules can be found, which are the most interesting ones [4,47,49]. Along the $a$-direction, the symmetric $a_{g}$ as well as the infrared-active $b_{3 u}$ modes of CA and TTF are observed whereas the first one is infrared active only due to the emv-coupling. Due to the electronic coupling to the charge-transfer band, we model each mode by a Fano function (Equation (A8)). In the case of the $b_{3 u}$ resonance, the vibrational frequency appears below $700 \mathrm{~cm}^{-1}$ and thus outside the studied regions.

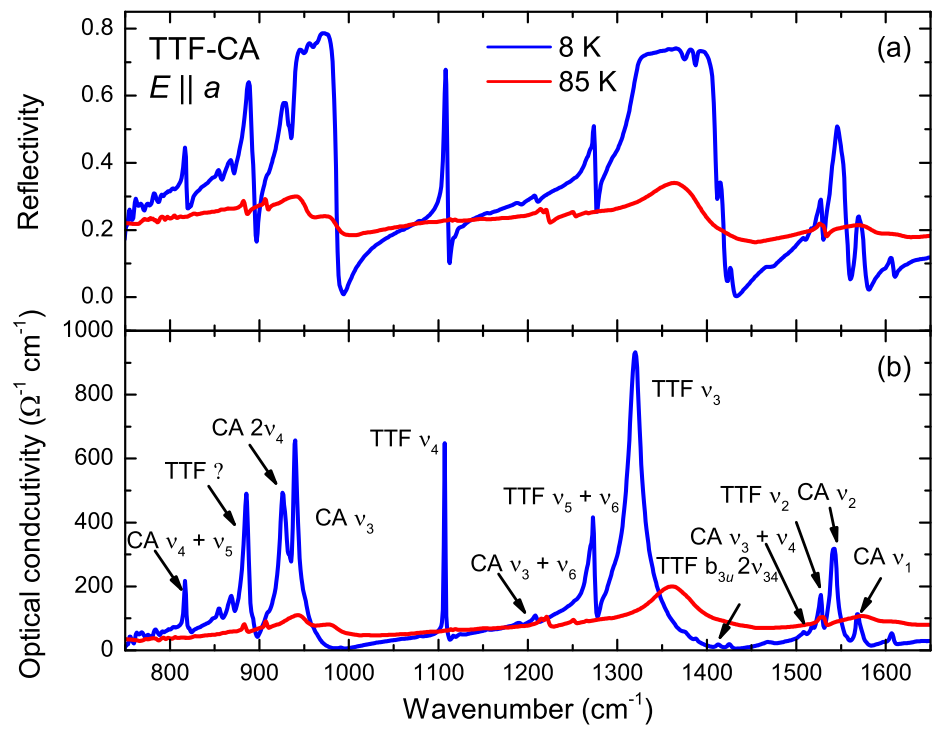

Figure 18. (a) Reflectivity of TTF-CA for $T=8 \mathrm{~K}$ (blue) and $85 \mathrm{~K}$ (red) measured with $E \| a$ between $750 \mathrm{~cm}^{-1}$ and $1650 \mathrm{~cm}^{-1}$; (b) Corresponding optical conductivity is illustrated. The maximum is mainly caused by emv-coupled modes which gain intensity in the dimerized ionic phase.

Above the phase transition in the neutral phase, the TTF and CA molecules are not dimerized: the distance between them is similar $3.70 \AA$; for that reason, the ag modes are only slightly infrared-active and the optical conductivity is low. 
In the ionic phase, the point inversion symmetry is lost as the molecules become dimerized: this alters the intermolecular distance and the intensity of $\mathrm{a}_{g}$ modes is enhanced enormously. This extreme gain is seen as a fingerprint for the dimerization of the TTF and CA molecules, for the increase of the ionicity, and for the transition in the ionic phase related to that.

In an initial step, we assigned the observed features to the corresponding vibrational modes. Starting with the lowest energy, the first maximum is located at $816 \mathrm{~cm}^{-1}$ and can be ascribed to the combination of the $v_{4}+v_{5}\left(\mathrm{ag}_{\mathrm{g}}\right)$ modes. In the harmonic approximation, such coupling between different vibrations is not allowed due the selection rules. The strong interaction between the molecules in the ionic phase leads to a strong deformation of the potential energy surfaces resulting in anharmonicity, which lifts the selection rules. Beginning with symmetry considerations, the molecules are in the $D_{2 h}$ symmetry group in gaseous phase with eight irreducible representations, as the single molecules in the ionic and in the neutral phase occupy the Wyckoff-position 2a and 4e, respectively. Therefore, they have only the site symmetry $C_{1}$ as well as $C_{\mathrm{i}}$. To receive the actual symmetry of the molecular vibration within the crystal, the site symmetry must be correlated with $C_{2 h}$ symmetry of the unit cell. It is noteworthy that the symmetry group of the site position is a subgroup of the unit cell space group and the free molecule symmetry. Thus, the intramolecular vibrations have the following symmetry representatives $a_{g}, b_{g}, a_{u}$, and $b_{u}$, where only the last two ones are infrared-active. The $a_{g}$-symmetry of the $\mathrm{D}_{2 \mathrm{~h}}$ symmetry group stays conserved in this case. For combination modes as well as for overtones, the original modes with the symmetry $a_{g}$ lead, in the case of the direct product $a_{g} \otimes a_{g}$, again to an $\mathrm{a}_{\mathrm{g}}$ symmetric mode which stays by that symmetric and infrared-active via emv-coupling. In most cases, the intensity of the higher order modes is strongly reduced in comparison to the fundamental one. The resonance frequency is lower than the sum or product of the fundamental mode due to the anharmonicity of the potential energy surface.

The next strong feature is at $885 \mathrm{~cm}^{-1}$. An antisymmetric mode can be excluded as the intensity increases in the ionic phase and the spectral shape is antisymmetric, indicating an emv-coupled mode. From our calculations, we cannot assign it to a fundamental $a_{g}$ mode, combination mode or overtone with the corresponding resonance frequency. The satellite peaks at lower energy are very weak and do not appear in the neutral phase. Therefore, we also assign these features to emv-coupled vibrations. As an origin, symmetric modes with the $b_{1 g}, b_{2 g}$, and $b_{3 g}$ are also possible. Since these modes are in accordance to the symmetry of the unit cell, they can have the $\mathrm{ag}_{\mathrm{g}}$ symmetry and by that become visible along the $a$-direction.

Two modes in the range from $900 \mathrm{~cm}^{-1}$ to $1000 \mathrm{~cm}^{-1}$ are from the second harmonic of the $v_{4}$ and $v_{3}$ modes of the CA molecule. At $1100 \mathrm{~cm}^{-1}$ a very sharp feature is located which is not existing above $T_{\mathrm{NI}}$ and is attributed according to Table $\mathrm{C} 2$ to the symmetric $v_{4}\left(\mathrm{a}_{g}\right)$ mode of the TTF molecule. Between $1100 \mathrm{~cm}^{-1}$ and $1300 \mathrm{~cm}^{-1}$ several weak and narrow bands can be discovered. The strongest is settled at $1208 \mathrm{~cm}^{-1}$ and correlates with the combination mode of $v_{3}+v_{6}$ of CA. The combination mode of $v_{5}+v_{6}$ of TTF is located $1270 \mathrm{~cm}^{-1}$. The maximum with the highest intensity is caused by the $v_{3}$ mode of the TTF molecules located at $1320 \mathrm{~cm}^{-1}$. This is not surprising since the coupling parameter $g_{i}$ with $115 \mathrm{meV}$ is the largest. Close to this mode, several weak features are connected whereas one of them corresponds to the strongly infrared-active, odd $v_{34}\left(\mathrm{~b}_{3 u}\right)$ mode. At $1507 \mathrm{~cm}^{-1}$ the combined mode of $v_{3}+v_{4}$ of CA appears. The three subsequent features at $v=1527 \mathrm{~cm}^{-1}, 1540 \mathrm{~cm}^{-1}$, and $1570 \mathrm{~cm}^{-1}$ accord to the $v_{2}$ of TTF, $v_{2}$ of CA and the $v_{1}$ mode of CA. This trident-like structure is induced by the small coupling constant of the TTF's modes. From Table 2, it can be deduced that $g_{i}$ of CA decreases with rising frequency. By that, the intensity of the $v_{1}$ of $\mathrm{CA}$ is weaker than the intensity of the $v_{2}$ mode.

In the neutral phase, where the molecules are not dimerized, these modes are only very weak. Nevertheless, the strongest coupled modes can be identified, for example the $v_{3}$ mode of CA at $978 \mathrm{~cm}^{-1}$ or the $v_{3}$ of TTF at $1360 \mathrm{~cm}^{-1}$. It is interesting to note that not only intramolecular vibrations are coupled, but also the intermolecular Peierls mode can couple to the intramolecular modes via the 
modulation of the charge-transfer band. This was nicely demonstrated by Masino et al. [68] comparing Raman and infrared spectra.

Since several overtones and combination modes are found in the energy range below $1700 \mathrm{~cm}^{-1}$, we can assume that several weak features must appear above $1700 \mathrm{~cm}^{-1}$, which are depicted in Figure 19. Indeed, aside from the fundamental vibrations of the C-H bonds of TTF at $3100 \mathrm{~cm}^{-1}$ several asymmetric resonances occur between $1650 \mathrm{~cm}^{-1}$ and $3000 \mathrm{~cm}^{-1}$. The weakest resonance at $1783 \mathrm{~cm}^{-1}$ is the second harmonic of the $v_{3}$ mode of CA. The fundamental mode $v_{4}$ mode of TTF, which is situated at $1108 \mathrm{~cm}^{-1}$, causes an overtone at $2050 \mathrm{~cm}^{-1}$ as well. The broad feature at $2400 \mathrm{~cm}^{-1}$ originates from the ungerade $v_{1}$ vibration of the $\mathrm{CO}_{2}$ which is present in air. The asymmetric resonance located at $2714 \mathrm{~cm}^{-1}$ is associated with the overtone of the $v_{3}$ mode of TTF. As aforementioned, the strongest resonances above $3000 \mathrm{~cm}^{-1}$ are correlated to modes involving the $\mathrm{C}-\mathrm{H}$ bonds of TTF. The weak sidebands are assigned because of the low intensity to the second harmonic of the $v_{1}$ and $v_{2}$ modes of CA and the $v_{2}$ mode of TTF.

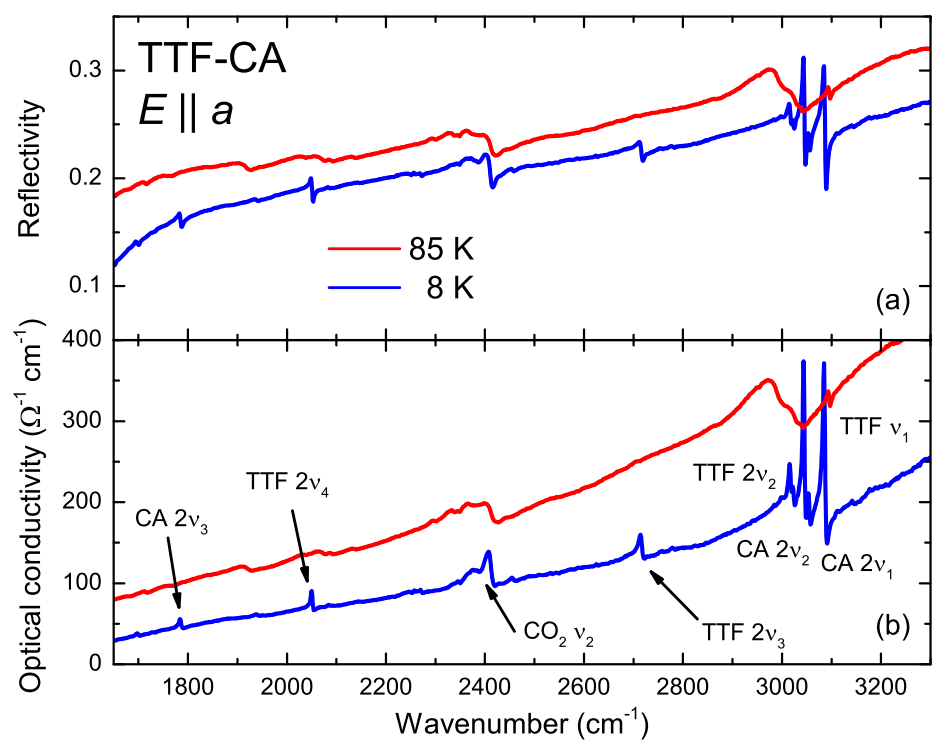

Figure 19. (a) Optical reflectivity of TTF-CA for $T=8 \mathrm{~K}$ (blue) and $85 \mathrm{~K}$ (red) measured along the a-direction; (b) Corresponding conductivity as a function of frequency for the same temperatures. The peaks at $3200 \mathrm{~cm}^{-1}$ originate from the $\mathrm{C}-\mathrm{H}$ vibration of the TTF molecule. The weak resonances mainly belong to higher harmonic vibrations of both molecules, CA and TTF.

\section{Photo-Induced Phase Transition in TTF-CA}

After having discussed the steady-state optical properties of TTF-CA, we now turn to the photo-induced non-equilibrium state. Over the decades, a large number of ultrafast pump-probe experiments have been performed on TTF-CA in order to explore the photo-induced phase transition (PIPT) that occurs in this one-dimensional charge-transfer compound, most of them confined to the femto- and picoseconds time range [35,58,69-80]. The studies were mainly restricted to low temperatures (typically $4 \mathrm{~K}$ ) or very close to the phase transition $\left(T \leq T_{\mathrm{NI}}\right)$. In most cases, the charge-transfer band [5] with its maximum located at $0.65 \mathrm{eV}$ was excited in these experiments while the probing range was predominant in the visible spectral range $[76,81]$. There are only very few investigations dedicated to the infrared spectral range [82-84].

All of these studies are based on the fact that the ionic-neutral phase transition can be induced by photo excitation in both directions: from the neutral phase to the ionic, and from the ionic phase to the neutral state. Since the phase transition is accompanied by a break of the symmetry (see Section 1 , Introduction), it can also be detected by structural resolving techniques. Indeed, Guérin et al. [77] performed a time-resolved X-ray diffraction experiment where they could detect the formation of 
a neutral phase in the ionic matrix. The photo-generated state was stable up to $2.5 \mathrm{~ns}$, demonstrating that the induced state is very robust and long-living.

The ionic-neutral, photo-generated phase transition in TTF-CA is the archetype for a PIPT, discovered by Koshihara et al. [69] a quarter of a century ago. Although numerous studies were undertaken since, there are still many open questions, for instance: What is the dependence of the PIPT on external parameters such as excitation wavelength, photon flux, and environmental temperature (between $T=4 \mathrm{~K}$ and $T_{\mathrm{NI}}$ )? What are the relaxation processes of the induced state/domains and the relaxation time? How does the infrared spectrum respond after photo excitation with a photon energy higher than $1.44 \mathrm{eV}$ ? In the present study, we concentrate on the micro- and millisecond time range and on the mid-infrared spectral range. For the excitation, laser pulses with a photon energy of $2.33 \mathrm{eV}$ were used.

\subsection{Underlying Principle}

In the original work of a photo-induced phase transition in TTF-CA, the crystal at $T=77 \mathrm{~K}$ was excited by a pulsed laser with an unspecified excitation energy [69]. At the same time, the reflectivity was probed perpendicular to the stacking direction, as visualized in Figure 20, in the spectral range from 1.5 to $4 \mathrm{eV}$ (corresponding to $12,000 \mathrm{~cm}^{-1}$ to $32,000 \mathrm{~cm}^{-1}$ ). The maxima at $3.3 \mathrm{eV}\left(26,600 \mathrm{~cm}^{-1}\right.$ ) and $2.5 \mathrm{eV}$ in the neutral phase can be assigned to the intramolecular excitation of $\mathrm{TTF}^{\rho}$. The maxima shift to lower energies was due to the increasing ionicity below $T_{\mathrm{NI}}$. A similar conclusion can be drawn from the consideration of the band structure, in which the distance of the bands below the valence band and the bands above the conduction is reduced below the phase transition (Figure 10 and discussion in Section 3). The excitations of the anion CA are above $4.0 \mathrm{eV}$. The modification of the optical reflectivity can be used to determine the present state of TTF-CA. A comparison of the modification of the reflectivity between the thermodynamic equilibrium above $(T=90 \mathrm{~K})$ and below (77 K) the transition with the photo-induced change of the reflectivity, indeed, reveals a transition from the ionic phase to the neutral one. The state decays within a few milliseconds. Measurements of the static photoconductivity suggest that neutral domains are generated, which are separated from the ionic phase by neutral-ionic domain walls (NIDW); and these NIDWs contribute with their fractional charge state to the photocurrent. The activation energy of the photocurrent [69] is $\Delta_{\text {Photo }}=0.09 \mathrm{eV}$ and corresponds exactly to the activation energy of the dark current. From that, we can deduce that in both cases the conductivity contributions are thermally activated or optically excited NIDWs, respectively.

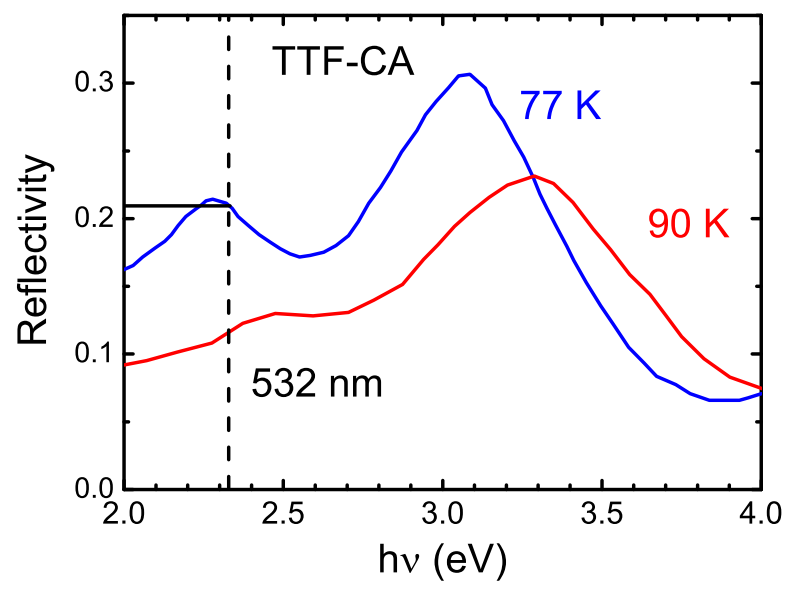

Figure 20. Reflectivity of TTF-CA in the ionic (blue) and neutral (red) phase. The dashed line marks the photon energy of the exciting laser. (Adapted from [69]).

This pioneer ingreport [69] triggered numerous studies [70,76,85-87] of the PIPT aiming to examine the creation process of the neutral domains in the ionic phase. Most of the studies were performed at two temperatures: either very close to the transition (approximately $78 \mathrm{~K}$ ) or for a very 
low temperature $(\sim 4 \mathrm{~K})$. It turned out that in the vicinity of the phase transition, the conversion efficiency is significantly larger at the same photon density. Beside temperature, the dependence of the PIPTs on the photon energy was examined in the near-infrared range. It was demonstrated that the laser pulses with a photon energy $E_{\text {Photon }}$ between $0.65 \mathrm{eV}$ and $1.55 \mathrm{eV}$ must exceed a certain threshold intensity $[85,86,88]$ to induce the neutral domains. The photon energy corresponds in this case to the energy $\hbar \omega_{\mathrm{CT}}$ of the charge-transfer band/exciton (CT) which we have identified in the spectrum as a broad maximum at $5200 \mathrm{~cm}^{-1}(0.65 \mathrm{eV})$. A successive decrease of $E_{\text {Photon }}$ beginning from $1.55 \mathrm{eV}$ leads to a decrease of the threshold intensity [86], which does not drop to zero $E_{\text {Photon. The }}$ situation changes when the intramolecular, vertical electronic transition of $\mathrm{CA}^{-}$and especially of $\mathrm{TTF}^{+}$ is excited. The four lowest electronic transitions in $\mathrm{TTF}^{+}$and $\mathrm{TTF}^{0}$ are listed in Table 3 causing four different bands in the visible spectral range which for TTF-CA in the ionic phase are located between $2 \mathrm{eV}$ and $4.5 \mathrm{eV}[66,89]$. From Table 3, it is apparent that the position strongly depends on the ionicity $\rho_{t}$. Therefore, these features can be used to determine the critical temperature of the phase transition.

Table 3. Electronic transitions of $\mathrm{TTF}^{+}, \mathrm{TTF}^{0}, \mathrm{CA}^{-}$, and $\mathrm{CA}^{0}$ correlated with the corresponding experimentally determined excitation energy in wavenumbers $\left(\mathrm{cm}^{-1}\right)$ and photon energy $(\mathrm{eV})$. The relative intensities are put in brackets.

\begin{tabular}{ccc}
\hline Transition & $\begin{array}{c}\text { Excitation Energy TTF }^{+}[90] \\
\text { in Acetonitril }\end{array}$ & $\begin{array}{c}\text { Excitation Energy TTF } \\
\text { in Hexan }\end{array}$ \\
\hline 1 & $17,300 / 2.14(0.27)$ & $22,200 / 2.76(0.02)$ \\
2 & $20,300 / 2.51($ weak) & $27,100 / 3.37(0.16)$ \\
3 & $23,000 / 2.85(1.00)$ & $31,600 / 3.92(0.89)$ \\
4 & $29,600 / 3.67(0.52)$ & $33,000 / 4.09(1.00)$ \\
\hline Transition & Excitation Energy CA ${ }^{-}[93,94]$ & Excitation Energy CA $\mathbf{C A}^{\mathbf{0}}[95]$ \\
\hline 1 & $22,300 / 2.77(-)$ & $27,200 / 3.37(-)$ \\
2 & $23,700 / 2.94(-)$ & $34,800 / 4.32(-)$ \\
3 & $31,100 / 3.86(-)$ & \\
\hline
\end{tabular}

In the case of TTF, the transition bands undergo a redshift between $3400 \mathrm{~cm}^{-1}$ and $8600 \mathrm{~cm}^{-1}$ in comparison to the gas phase. It should be noted, however, that due to the interaction of the molecules with their environment-for instance with neighboring molecules in the crystal or in a solvent-a so-called solvent shift or crystal shift [96] is induced that causes most of the interband transitions to move to lower energies. The magnitude of the shift depends on the type of solvent and affects the energy levels differently [38]. Thus, the listed values for the neutral and charged state of the molecules are solely guiding numbers and can vary. Figure 20 demonstrates that the temperature-dependent shift of the two maxima is about $1600 \mathrm{~cm}^{-1}$ in TTF-CA. This corresponds - under the assumption of a linear relation between the excitation energy and the molecular charge and the usage of Table 3-to a change of the molecular charge of $0.25 e$ and $0.2 e$, respectively, which is in perfect agreement with values derived from the resonance frequency of the $v_{10}\left(\mathrm{~b}_{1 u}\right)$ mode.

If the second harmonic of a pulsed Nd:YAG laser is used in PIPT measurements, i.e., an excitation frequency of $19,000 \mathrm{~cm}^{-1}$, corresponding to $2.35 \mathrm{eV}$, only one of the first transitions of $\mathrm{TTF}^{+}$is excited, because all intermolecular transitions of $\mathrm{CA}^{-}$lie above that photon energy (Table 3). In principle, excitations from lower lying bands into the valence band are also possible; however these are not included here. Suzuki et al. [85] compared the dependence of the conversion efficiency on the photon energy (see Figure 26 below) and found that by excitation of intramolecular transitions, no threshold intensity occurs to create neutral domains.

In Figure 21, the optical generation of neutral domains is schematically illustrated. Panels (b) and (c) show how in the case of the intramolecular excitations, Frenckel-excitons $[38,96]$ are created; i.e., electron-hole pairs that are strongly localized on the excited molecules in contrast to Wannier-excitons 
that occur in anorganic semiconductors such as silicon. The Frenckel-excitons decay via various decay channels into several charge-transfer excitons [74] and by that lead to a phase transition, as depicted in panels (d) and (e). The participating processes are illustrated in more detail in Figure 22. The excitation by a photon of a certain energy leads to a vertical transition according to the Franck-Condon principle. The excited electron can interact with the remaining hole by Coulomb attraction, alternating the surrounding electronic system and orbitals. This takes place in the first few femtoseconds. One route back is decay and recombination of the electron-hole (exciton) pair, which leads to a direct relaxation into the initial state. Alternatively, the exciton can break up and create several low-lying excited states, for instance charge-transfer excitons or collective excitations, by many-body scattering mechanisms or Auger decay leading to a cooperative effect. In addition, energy is dissipated and the electronic system couples to the underlying lattice by which energy is transferred and phonons are excited. They appear as damped oscillations in the time-dependent signal of pump-probe experiments. However, the low-lying excited states trigger the phase transition by relaxation to the hidden "false ground" state.

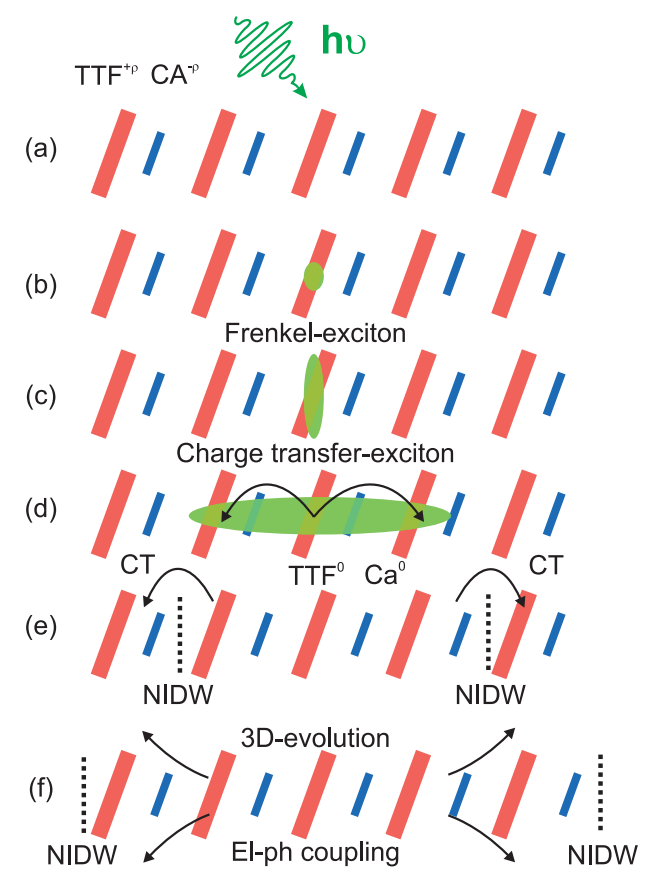

Figure 21. (a) Illustration of a one-dimensional chain of dimerized $\mathrm{TTF}^{+\rho}$ and $\mathrm{CA}^{+\rho}$ pairs in the ionic phase in TTF-CA. $\mathrm{TTF}^{+} \rho$ molecules are excited with a laser pulse of the photon energy $E_{\text {Photon }}=h v$; (b) Vertical excitation of the HOMOs of $\mathrm{TTF}^{+} \rho$ according to the Franck-Condon-principle in the LUMO of $\mathrm{TTF}^{+} \rho$. The excitation is strongly localized on the molecule; (c) Creation of excitons, for instance Frenkel-excitons, which are delocalized across the whole molecules; (d) Via different relaxation processes and channels, charge-transfer excitons are created that trigger the transition of the neutral phase; (e) The dimerization is suppressed and the charge between the molecules is redistributed. A neutral domain is created in the ionic host matrix, which is separated by NIDWs; (f) Afterwards, the neutral domains extend along the one-dimensional chain. By electron-phonon-coupling, neighboring ionic chains are also converted into neutral regions and a three-dimensional domain is established.

A so-called "over-neutralized" state generated in the relaxation process well after the pulsed photo excitation is also seen by time-resolved structural studies [97]. The created metastable domain can further proliferate with a long life time and finally decays back to the equilibrium ground state across the energy barrier. The life time can be on the nanosecond, but also on the millisecond time range. 


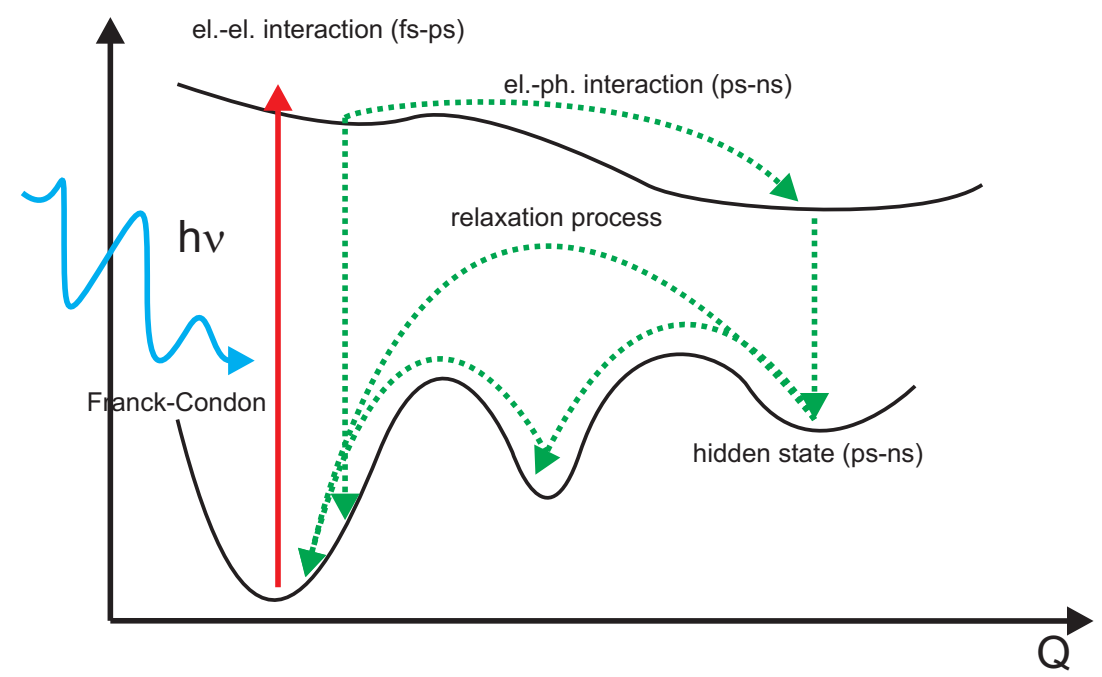

Figure 22. Sketch of the generation of a hidden metastable state by photons with a certain energy. The potential energy surface is shown for the ground and excited states (black solid lines) for a certain order parameter $Q$. The blue arrow symbolizes a photon with the energy $h v$ exciting vertically the ground state into a higher energy level. Different relaxation channels (green dotted lines) are possible which can result in a metastable hidden state with a different value of the order parameter from the ground state. It is separated from the initial state by energy barriers.

The process described above generates several charge-transfer excitons, in contrast to direct excitation of the charge-transfer band, where only one charge-transfer exciton is created; this is not enough to establish a macroscopic, metastable domain extended over several $\mathrm{D}^{0} \mathrm{~A}^{0}$ pairs. It takes a sufficient number of photons that create a charge-transfer exciton in order to establish a multiplicative, non-linear effect and eventually form metastable domains. By the generation of the one-dimensional, neutral, non-dimerized region, domain walls are formed between the neutral and ionic parts (NIDW). The excitation energy of the NIDW is about $0.1 \mathrm{eV}[9,11]$ and corresponds to the activation energy of $0.12 \mathrm{eV}$ and $0.065 \mathrm{eV}$ in the ionic phase, as we will determine below.

In the generation process of NIDWs, the excitation energy plays an important role besides the excitation wavelength [76], because soliton states with a spin can also be created. Calculations suggest that the activation energy is between $25 \mathrm{meV}$ [22,23] and $56 \mathrm{meV}$ [24]. Soos and Painelli [24] suggested to use the term "domain" only when the total length exceeds 30 donor-acceptor pairs; in the following we will apply it also to five and more pairs.

Finally, the electronic system couples to the underlying lattice and excites phonons [87] via electron-phonon coupling; i.e., shock-waves are generated. They can convert neighboring chains into neutral domains (Figure 21f). The total conversion process is finished after several hundred picoseconds. In ultrafast pump-probe experiments, it was observed, however, that the life time can extend up to the millisecond time range.

\subsection{Experimental Configuration}

Our time-resolved studies are performed on TTF-CA single crystals that were grown as described in Section 2. The specimens were characterized by X-ray, dc-transport and standard infrared spectroscopy (Section 4). For optical excitation, we use the second harmonic light pulse $(\lambda=532 \mathrm{~nm}$, pulse length: $8 \mathrm{~ns}$ ) of an Nd:YAG laser. The time-dependent response of the infrared reflectivity signal is detected by a Bruker Vertex 80v Fourier-transform spectrometer operating in the step-scan mode as described in detail in Refs. [83,84]. A sketch of the experimental setup is given in Figure 23. The samples are cooled down by a CryoVac helium-flow cryostat that is placed in a Bruker Hyperion infrared microscope. 


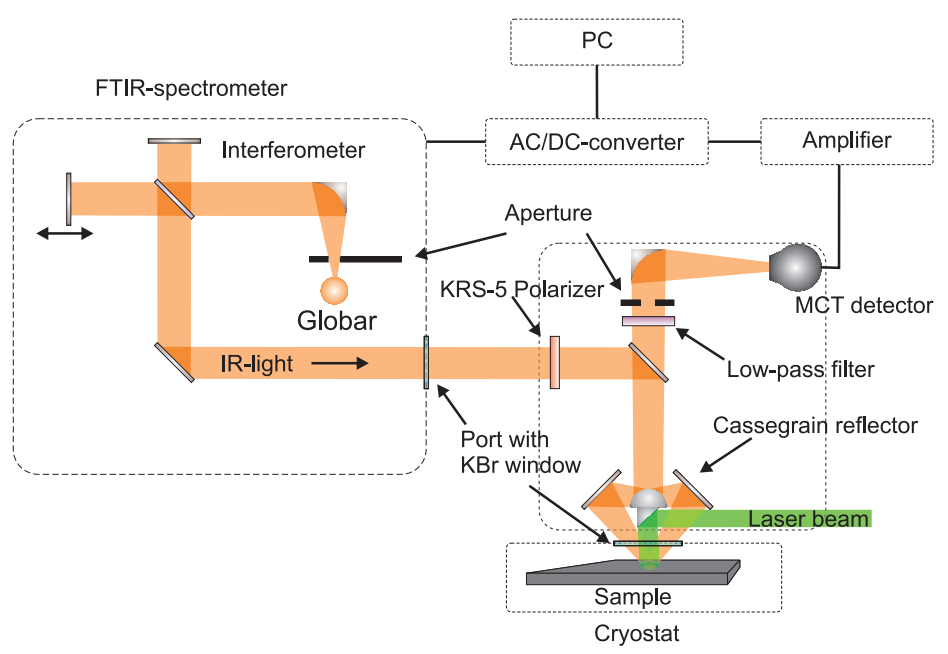

Figure 23. Sketch of the optical setup including the laser beam to excite the TTF-CA sample. The setup consists of three main parts (dashed lines). The centerpiece of the setup is the Fourier-transform infrared spectrometer containing the electronics as the A/D-converter and amplifier, but also the Globar light source, the interferometer with the beamsplitter and several mirrors to deflect the light beam. The second part is the infrared microscope which is attached to the spectrometer. There, the light is focused on the sample by a $15 \times$ Cassegrain reflector. Furthermore, a polarizer and bandpass filter can be mounted in the microscope. The MCT detector is placed at the end of the light beam. Adapted from Peterseim and Dressel [84].

\subsection{Photo-Induced Phase Transition}

The static reflectivity of TTF-CA for $E \| a$ in the ionic and neutral phase ( $T=85 \mathrm{~K}$ and $79 \mathrm{~K}$ ) is presented in Figure 24a. As discussed in Section 4.2, the symmetric ag modes become infrared activated in the ionic phase due to the dimerization of the molecules along the $a$-direction. This effect is a unique property of the ionic phase; thus it will be utilized to measure the presence of the ionic state and how it vanishes with time [87].

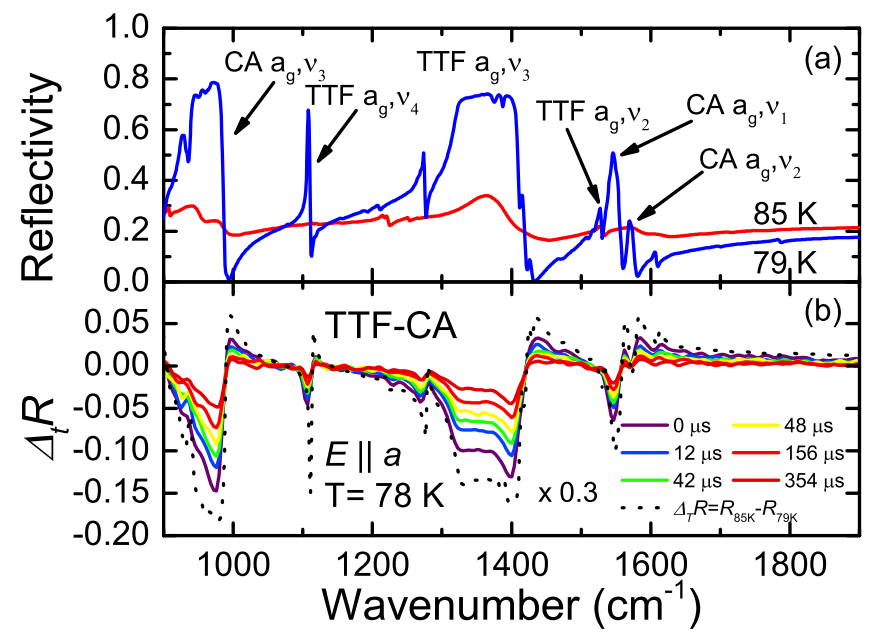

Figure 24. (a) Reflectivity of TTF-CA along the stacking direction in the mid-infrared frequency range for $T=79 \mathrm{~K}$ (red) and $85 \mathrm{~K}$ (blue). The ag modes become strongly enhanced due to the dimerization of the TTF and CA pairs in the ionic phase; (b) Temporal sequence of the reflectivity change $\Delta_{t} R=R(t)-R(0)$ (solid lines) after photo excitation depicted for various delay times and directly compared to the static reflectivity difference $\Delta_{T} R=R_{85 \mathrm{~K}}-R_{79 \mathrm{~K}}$ (dashed line). Adapted from [83]. 
In Figure 24a, the time-dependent behavior of the reflectivity change $\Delta_{t} R=R(t)-R(0)$ at $T=78 \mathrm{~K}$ and for a laser pulse intensity of $0.71 \mathrm{~mJ} / \mathrm{cm}^{2}$ is directly compared with the variation of the static reflectivity upon passing through neutral-ionic phase transition $\Delta_{T} R=R_{85 \mathrm{~K}}-R_{79 \mathrm{~K}}$. By photo excitation $\Delta_{t} R$ becomes negative within a short time that is below the experimental time resolution of $6 \mu$ s. The direct comparison of the $\Delta_{t} R$ shape and the static reflectivity change $\Delta_{T} R$ reveal that the ionic phase was not only dissolved, but also a transition into a neutral state was induced. Within several hundreds of microseconds, the signal $\Delta_{t} R$ relaxes back to zero which means that the ionic phase is reestablished. Moreover, no change of the spectral shape with the elapsed time and laser pulse intensity could be detected.

$\Delta_{T} R$ was scaled by a factor of 0.33 to reach the same value as the maximum value of $\Delta_{t} R$ immediately after the photo excitation. The difference of the absolute values originates from the different penetration depth of the visible light (approximately $200 \mathrm{~nm}$ ) compared to infrared light. The penetration depth was determined from the reflectivity in the visible range depicted in Figure 20 by fitting the spectra with three Lorentz functions. From the optical conductivity, $\sigma_{1}(v)$ and $\sigma_{2}(v)$, the penetration depth $\delta_{d}$ can be deduced from equation [98]

$$
\delta_{\mathrm{d}}(\omega)=\sqrt{\frac{2}{\omega \nu_{0}}}\left[\left(\sigma_{1}^{2}(\omega)+\sigma_{2}^{2}(\omega)\right)^{2}+\sigma_{2}(\omega)\right]^{-0.5}
$$

In the infrared range, the penetration depth is ten times larger than in the visible spectral range; thus our experiments probe a considerable volume.

To trace the temporal evolution of the PIPT in dependence of the pump intensity and the sample temperature, we have chosen the very intense $v_{3}\left(\mathrm{a}_{\mathrm{g}}\right)$ mode of TTF residing at $1390 \mathrm{~cm}^{-1}$ since we have asserted that the temporal evolution is the same for the entire spectra. The normalized $\Delta_{t} R(t)$ is represented in Figure 25a for different pulse intensities. We can recognize several temporal regimes: at the beginning the signal decays very fast but after some time it flattens out. In the vicinity of $T_{\mathrm{NI}}$ the first component decays faster with reduced laser intensity. In literature, the time-dependent signal is commonly fitted by a simple single or double-exponential function [74]. However, in the present case, it only can be nicely modeled by a stretched-exponential function, which is also called Kohlrausch-William-Watt function $\Delta_{t} R(t) \propto \exp \left\{-(t / \tau)^{\beta}\right\}$, as depicted in Figure 25a,b. The fitting parameters $\beta$ and $\tau$ are a function of the laser intensity and decrease from 0.35 to 0.42 and from $3.4 \times 10^{-5}$ to $2.4 \times 10^{-4} \mathrm{~s}$ with decreasing laser intensities. Since the behavior of the PIPT in a larger temperature range below $T_{\mathrm{NI}}$ is of great interest, the temporal behavior was examined at a temperature between $68 \mathrm{~K}$ and $78 \mathrm{~K}$.

In Figure 25b, $\Delta_{t} R(t)$ is displayed for $T=68 \mathrm{~K}, 73 \mathrm{~K}$, and $78 \mathrm{~K}$. Far below the neutral-ionic transition temperature $T_{\mathrm{NI}}$, the temporal dynamics of the reflectivity drops very fast within the first $20 \mu \mathrm{s}$ and approaches asymptotically a constant value; this behavior is in contrast to the temporal profile at the vicinity $(T=78 \mathrm{~K})$ of $T_{\mathrm{NI}}$, which constantly diminishes. Similar to the dependence of the fitting parameters on the laser intensity, the effective recombination time $\tau$ decrease from $2.4 \times 10^{-4} \mathrm{~s}$ to $3.2 \times 10^{-6} \mathrm{~s}$ and the stretching exponent $\beta$ from 0.42 to 0.23 , respectively, with decreasing sample temperature.

The total change of $\Delta_{t} R(0)$ is depicted on a logarithmic scale for the normalized temperature $\left|T / T_{\mathrm{NI}}-1\right|$ and for various laser intensities in Figure 25c. As typically for a PIPT [74], $\Delta_{t} R(0)$ diverges closely to the phase transition temperature and decreases exponentially, with no dependence on the laser intensity. The temperature dependence behavior of $\Delta_{t} R(0)$ is in accordance with previous experiments [76]. The actual absolute values can be higher since the first nanoseconds of the phase transition are not captured due to the limited time resolution. 

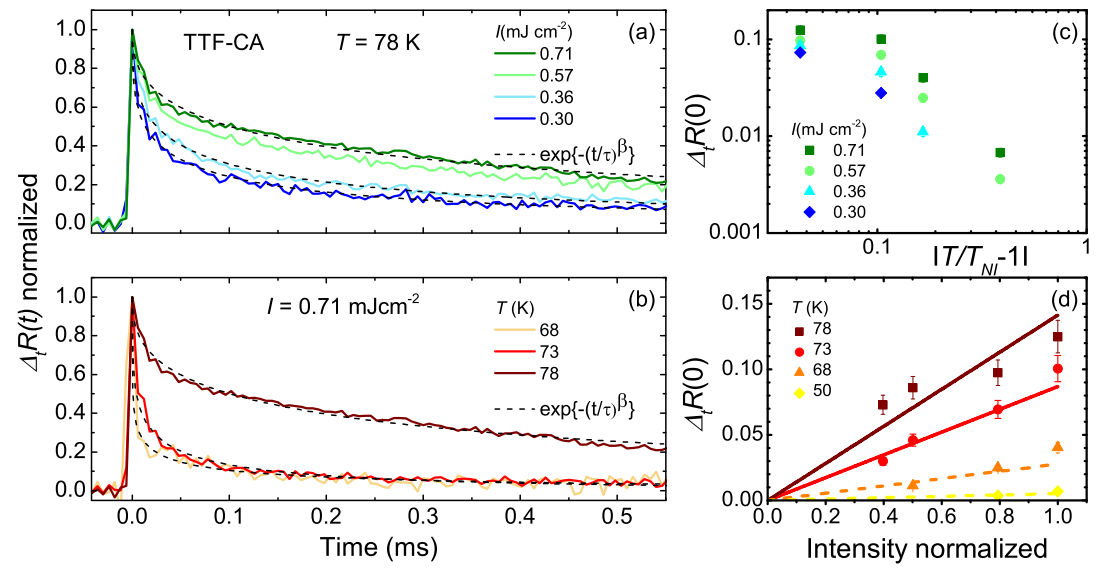

Figure 25. (a,b) Normalized $\Delta_{t} R(t)$ for various laser intensities recorded at $v=1390 \mathrm{~cm}^{-1}$ for $T=78 \mathrm{~K}$ and for different temperatures for $I=0.71 \mathrm{~mJ} / \mathrm{cm}^{2}$. The time profile can be successfully fitted by a Kohlrausch-Williams-Watt (KWW) function $\exp \left\{-(t / \tau)^{\beta}\right\}$ (dashed lines); (c) Absolute value of $\Delta_{t} R$ for different laser intensities and temperatures observed immediately after the photo excitation; (d) $\Delta_{t} R$ as a function of the normalized laser intensity for temperatures between $50 \mathrm{~K}$ and $78 \mathrm{~K}$. Adapted from Ref. [83].

$\Delta_{t} R(0)$ is a linear function of the laser intensity, as visualized in Figure 25d. We do not recognize any saturation of the signal and no threshold value for the laser intensity applied. Therefore, a thermally induced phase transition can be excluded in general.

A comparison with previous measurements, which are presented in Figure 26, reveals that the observed linear behavior of $\Delta_{t} R(0)$ agrees very well with the study of Suzuki et al. [85]. There, the change of reflectivity was measured at $24,200 \mathrm{~cm}^{-1}$. In Figure 26, one sees that when the charge-transfer band is excited by light with an excitation wavelength of $\lambda=1064 \mathrm{~nm}$, a threshold intensity has to be exceeded to initialize the multiplication effect.

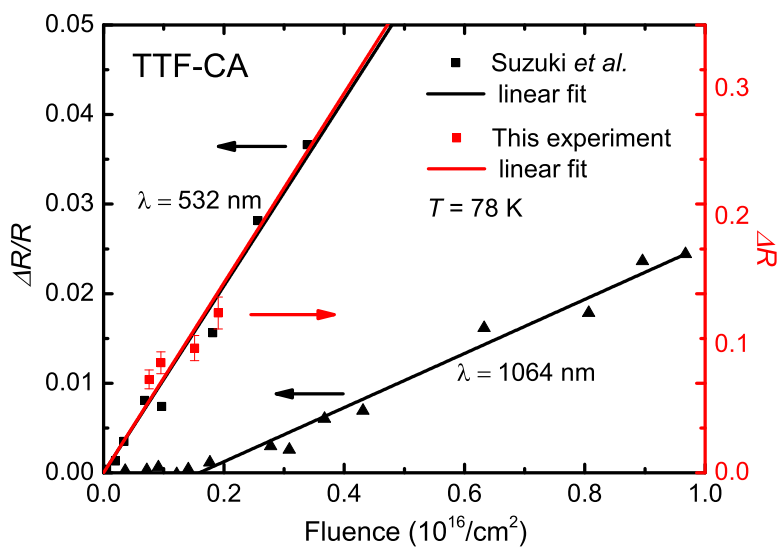

Figure 26. Comparison of the results from our study recorded at $v=1390 \mathrm{~cm}^{-1}$ and $T=78 \mathrm{~K}$ with an excitation energy of $2.33 \mathrm{eV}(\lambda=532 \mathrm{~nm})$ with the variation of the reflectivity at $24,200 \mathrm{~cm}^{-1}$ from Ref. [85] recorded at $3 \mu$ s after the photo perturbation was also detected at $T=78 \mathrm{~K}$ for two different excitation energies $(\lambda=532 \mathrm{~nm}$ and $1064 \mathrm{~nm})$.

From the laser beam penetrated volume $V=\pi r^{2} \delta_{d}(532 \mathrm{~nm}) \approx 5.6 \times 10^{-14} \mathrm{~m}^{3}$, the number of excited dimers $N_{\text {Dimer }}=2 \mathrm{~V} / V_{\text {Unit cell }}=1.4 \times 10^{15}$ can be calculated via the volume of the unit cell $V_{\text {Unit cell }}=812 \times 10^{-30} \mathrm{~m}^{3}$. The factor of 2 originates from the fact that two dimers are in the unit cell. The laser pulse with the highest intensity $I_{L}$ yields a photon number of $N_{\text {Photon }}=\pi r^{2} I_{\mathrm{L}} / E_{\text {Photon }}=E_{\mathrm{p}} / E_{\text {Photon }}=5.4 \times 10^{12}$ with the pulse energy $E_{p}$ and the photon energy 
$E_{\text {Photon}}$. This results in $25 \mathrm{D}^{+\rho_{\mathrm{t}}} \mathrm{A}^{-\rho_{\mathrm{t}}}$ transferred dimer pairs per photon. This corresponds exactly for a photon energy of $E_{\text {Photon }}=2.33 \mathrm{eV}$ and a conversion energy of $0.09 \mathrm{eV}$ per dimer which perfectly agrees with the energy to generate a NIDW.

\subsection{Heating Effect}

To exclude that thermal effect causes the phase transition, we have applied various thermodynamic models to determine the impact of our laser energy and a thermally induced transition. In order to make sure that no heat is accumulated, we have chosen a low repetition rate of only $20 \mathrm{~Hz}$, i.e., by a factor of 50 smaller than used in comparable experiments [99]; furthermore, the selected laser intensities are five times smaller. To make a precise prediction of the sample temperature, the models have been refined and only the penetrated volume was considered, instead of the whole sample volume, which is oversimplified but frequently applied to determine the sample temperature after photo excitation.

In a first step, the absolute temperature variation is calculated by neglecting any temperature dependence to receive an upper limit of the sample surface temperature. The temperature rise can be determined from the thermodynamic equilibrium equation, assuming that the entire laser pulse energy is converted into thermal energy:

$$
E_{\mathrm{p}}=n \int_{T_{0}}^{T} C_{\mathrm{p}}(T) \mathrm{d} T
$$

with $C_{p}(T)$ the temperature-dependent heat capacity in units of $\mathrm{J} /(\mathrm{mol} \cdot \mathrm{K}), E_{p}$ is the pulse energy, and $n=\delta_{\text {therm }} A \rho_{m} / M_{\text {mol }}$ the amount of substances that is warmed up in units of mol. It was assumed that the total energy is absorbed at the surface. $n$ is a function of the thermal diffusion length $\delta_{\text {therm }}=\sqrt{t_{p} \lambda_{\text {therm }} M_{\text {mol }} /\left(\rho_{m} C_{\mathrm{p}}\right)}$, which is on the scale of micrometers, with the irradiated area $A=0.28 \mathrm{~mm}^{2}$, the density $\rho_{m}$, the molar mass $M_{\text {mol }}$ of TTF-CA and $t_{\mathrm{p}}=6 \mu$ s after the time point the laser pulse hits the sample surface. As a value for the thermal conductivity, we use $\lambda_{\text {therm }}=0.02 \mathrm{~W} / \mathrm{cmK}$ taken from other charge-transfer salts [100]. The inset of Figure 27 displays the results from Equation (8). The relative temperature change $\Delta T$ after the irradiation of the sample with the laser energy $E_{p}$ increases from $\Delta T=1.2 \mathrm{~K}$ at $T=80 \mathrm{~K}$ to $\Delta T=1.6 \mathrm{~K}$ at a base temperature of $50 \mathrm{~K}$. Most importantly, the temperature always stays below the transition temperature $T_{\mathrm{NI}}=81.5 \mathrm{~K}$.

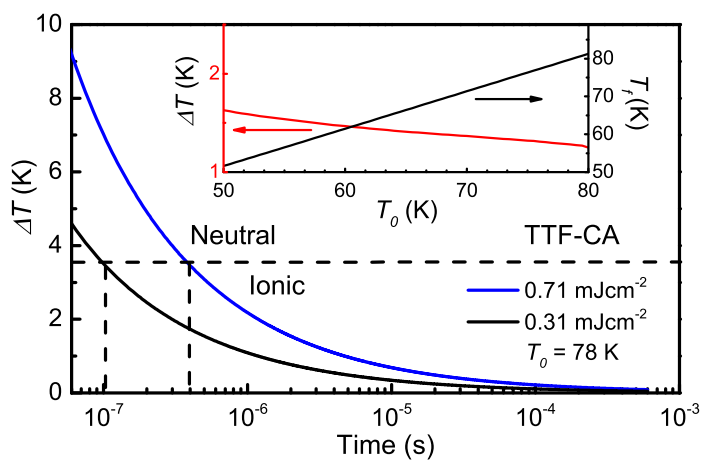

Figure 27. Time-dependent behavior of the surface temperature of TTF-CA, determined from Equation (9), after irradiation with the lowest and highest laser intensity at $T_{0}=78 \mathrm{~K}$. The surface temperature drops below $T_{\mathrm{NI}}$ after $500 \mathrm{~ns}$ and remains low (highlighted by the black dashed vertical line). Therefore, any thermally induced effect can be excluded as the origin of the made spectral observation since it would be far below our time resolution. The detail shows the absolute change (black) and the relative (red curve) temperature modification, $T_{f}$ and $\Delta T$, below $T_{\mathrm{NI}}$ derived from Equation (8), respectively. Adapted from [83]. 
The time-dependent evolution of the temperature was theoretically studied comprehensively by Bechtel [101]. An exact description of the equation can be found in Appendix D. When solely the sample surface was heated up by the laser beam, the change of the surface temperature $\Delta T(t)$ can be derived from the following equation:

$$
\Delta T(t)=\frac{2 Q_{\mathrm{p}}(1-R)}{\lambda} \frac{\left(\kappa \tau_{\mathrm{p}}\right)^{0.5}}{\pi^{0.5}}\left(\left(t \tau_{\mathrm{p}}^{-1}\right)^{0.5}-\left(t \tau_{\mathrm{p}}^{-1}-1\right)^{0.5}\right)
$$

We know from Equation (7) that the penetration depth $\delta_{d}$ for $h v=2.33 \mathrm{eV}$ is about $200 \mathrm{~nm}$ which is much smaller than the sample thickness of $200 \mu \mathrm{m}$. Therefore, we can assume that solely the surface is heated up. From Figure 20, the reflectivity $R=0.21$ at $2.33 \mathrm{eV}$ was determined. The thermal diffusivity was accordingly derived from the equation:

$$
\kappa=\frac{\lambda_{\text {therm }}}{\rho_{m} C_{\mathrm{p}}\left(T_{0}\right)} .
$$

Further parameters are the laser pulse width of $\tau_{\mathrm{p}}=8 \mathrm{~ns}$ and the radiant photon flux $Q_{p}$.

The temporal evolution of $\Delta T(t)$ decays with $\sqrt{t}$, as displayed in Figure 27 for $T$ close to $T_{\mathrm{NI}}$. Under the assumption that the complete pulse energy is converted in heat directly at the surface, the surface temperature shoots up across $T_{\mathrm{NI}}$. Afterwards, it drops within $500 \mathrm{~ns}$ below the transition temperature. Since a thermal induced transition would recover within a four order of magnitude faster time scale, we exclude that solely a heating effect is responsible for the spectral modification.

Furthermore, we also varied the laser intensity $Q_{p}$ by more than a factor of three and $\lambda_{\text {therm }}$ between $0.12 \mathrm{~W} / \mathrm{Kcm}$ (value for TTF-TCNQ [102] at $40 \mathrm{~K}$ ) and $0.001 \mathrm{~W} / \mathrm{Kcm}$ (for $\alpha$-(BEDT-TTF) ${ }_{2} \mathrm{I}_{3}$ [103] in the charge ordered phase) in Equation (9). However, we cannot observe any significant changes in the temperature drop which can be correlated with our measured data.

Going one step further, on the basis of our reflectivity data and its temperature-dependent behavior at $1390 \mathrm{~cm}^{-1}$ we calculated the reflectivity change in dependence of the temporal evolution of the temperature variation. In Figure 28, the experimental observed change of the reflectivity is compared with the calculated thermally induced one. It is obvious that the thermally induced transition signal would vanish within $500 \mathrm{~ns}$, whereas our detected signal persists for at least several hundreds of microseconds.

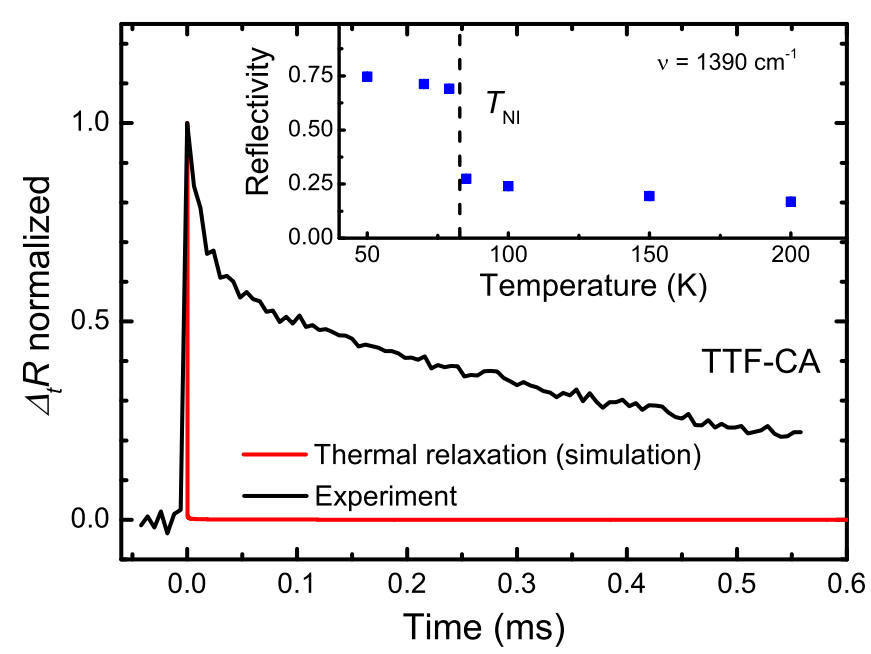

Figure 28. Comparison of the thermally-induced reflectivity change $\Delta_{t} R$ (red) with the observed photo-induced reflectivity change (black). Their decay characteristics are completely different from each other. In the inset, the reflectivity of the $v_{3}$ mode at $1390 \mathrm{~cm}^{-1}$ is displayed as a function of temperature. The reflectivity jumps at $T_{\mathrm{NI}}$ due to the enhanced infrared intensity of the emv-coupled mode in the ionic state (after [83]). 
Concluding, the temporal dynamic cannot be explained by a simple cooling mechanism. Furthermore, it is suggested that the initialization and relaxation process are of electronic origin.

\section{One-Dimensional Random Walk: Electronically Driven Transition}

The concept of an electronically induced phase transition [104] has already been introduced in Section 5 above, where we suggested that the creation of a domain can be considered as the excitation of neutral-ionic domain-wall (NIDW) pairs, which are the lowest excitation state in TTF-CA. Based on this concept, we now assume that after the NIDWs are generated, they perform a one-dimensional random-walk and annihilate after two NIDWs meet each other.

This process is analog to the creation and annihilation of soliton-anti-soliton pairs in trans-polyacetylene and MX chain compounds $[105,106]$. The two possible binding configurations of the ground state of trans-polyacetylene are depicted in Figure 29; both arrangements are energetically degenerated. The photon-generated soliton pairs can move freely along the chain as long as no traps or impurities capture them. However, when a solition meets an anti-soliton, they recombine. By applying a voltage, a photocurrent can be detected $[107,108]$ that decays nonlinearly and not exponentially, and provides information about the transport mechanism. Time-dependent optical absorption measurements yield further information of the lifetime of the generated particles [109]. There, the temporal evolution of the measured quantities can be described theoretically by a one-dimensional random-walk model that includes an annihilation process. With increasing temperature $T$, the photocurrent as well as the absorption signal decay faster, since with rising kinetic energy the probability to find a recombination partner increases. The same argument holds for increasing the laser intensity, because with a higher density of solitons the possibility for annihilation becomes larger.

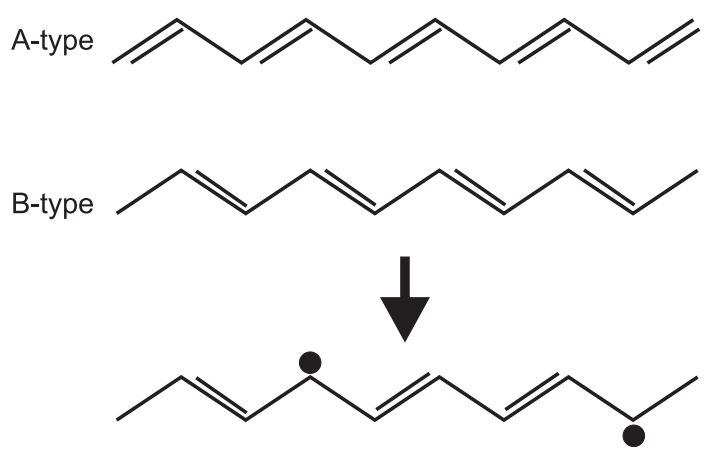

Figure 29. A- and B-type of trans-polyacetylene. The ground state is degenerated. By photon excitation, solitons and anti-solitons are created in the polymer chain which move along the chain and annihilate when they meet each other.

The diffusion random-walk annihilation process of equal particles, which we consider in the present case as the NIDWs, was theoretically studied for finite, closed and infinite chains. For a detailed description, we refer to the Refs. [106,110-114]. In the theoretical consideration, the most interesting parameter is the survival probability $S(t)$, which follows a $1 / \sqrt{t}$ evolution in an infinite chain. For a large chain, there exists a crossover from a power law of $S(t)$ to an exponential behavior of $S(t)$ for small finite rings. To model the temporal dynamics observed in our experiments and presented in Figure 25, a random-walk annihilation process is modeled on a closed ring that consists of $M=5 \times 10^{4}$ sites, and determined $S(t)$. For that we applied the direct method described in detail by ben Avraham [115]. Any three and two-dimensional interactions with the neighboring chains, traps as well as external fields were neglected at this point.

The average distance $L(0)$ is a function of $1 / N(0)$, where $N(0)$ is the initial randomly distributed number of NIDWs on the closed ring. Immediately after the creation process, $L(0)$ has the size of the neutral domains. We averaged over ten simulations in order to decrease the noise level in $S(t)$, 
especially for $L(0)>100$, since the reaction is very low for each time step. The probability for the NIDWs to jump to one of the neighboring sites was set to $50 \%$. When two NIDWs occupy the same site, they annihilate and are removed from the simulation. Figure 30 illustrates the corresponding time evolution schematically. The survival probability $S(t)$ is defined as follows:

$$
\frac{L(t)}{L(0)}=\frac{N(t)}{N(0)}
$$

Here, $S(t)$ corresponds to the time-dependent reflectivity change $\Delta_{t} R(t)$.

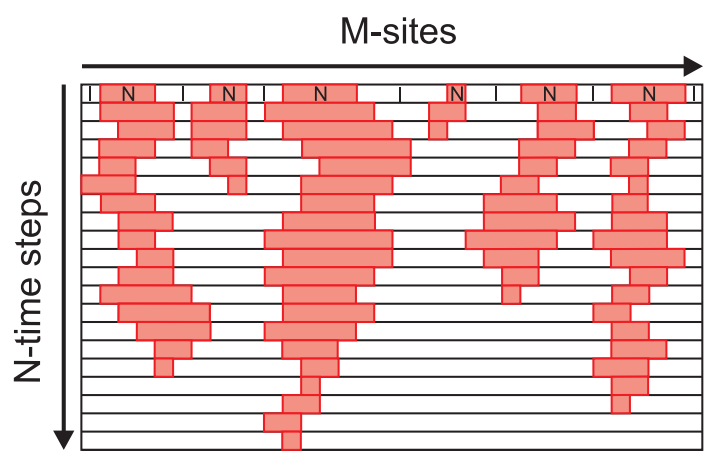

Figure 30. Illustration of a random-walk annihilation process of neutral-ionic domain walls (NIDW) along a one-dimensional chain with the length $M$. Neutral domains (red) are embedded in the ionic phase (white) separated from each other by NIDWs. After several time steps, the neutral domain collapses which is the same for the annihilation of two NIDWs.

In Figure 31a, $S(t)$ is displayed as a function of the experimentally adjusted time scale for various initial domain sizes $L(0)$. By comparing the simulated decay profile of $S(t)$ and $\Delta_{t} R(t)$ with experimental ones, it is obvious that they agree very well. The lifetime of initially large domains is long since $S(t)$ drops slowly, whereas small domains decay faster because the NIDWs' annihilation process takes place on a shorter time scale since the spacing between the NIDWs is smaller. Similar to $\Delta_{t} R(t)$, a KWW function models the temporal profile of $S(t)$ excellently, as depicted in Figure 31a.
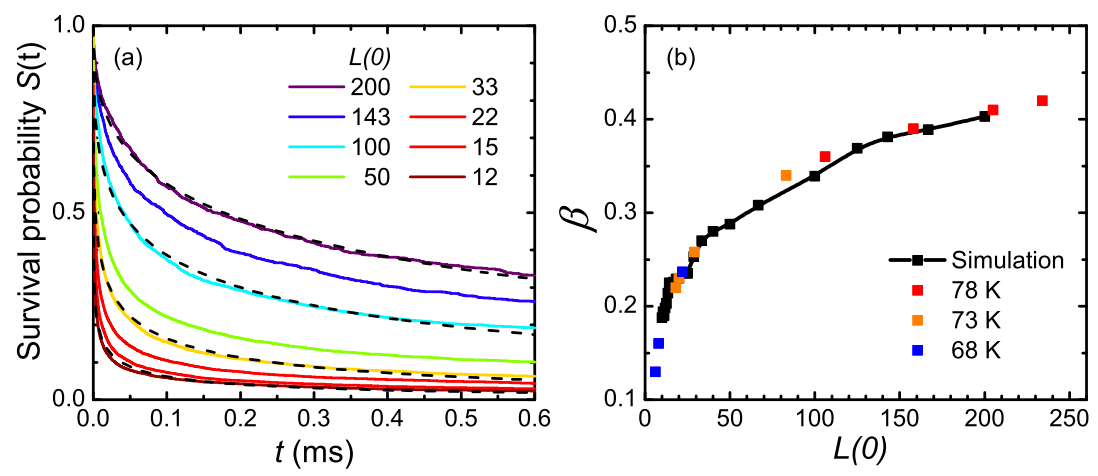

Figure 31. (a) Survival probability $S(t)$ of a domain wall pair on a one-dimensional chain as a function of time for different average domain sizes $L(0)$. The signal can be very well modeled by a Kohlrausch-Williams-Watt function (dotted line); (b) Fit parameter $\beta$ as a function of $L(0)$ derived from the fit of the KWW function to the simulated (black lines and dots) and the experimental $S(t)$ curve (colored squares) (After [83]).

In Figure 31, the experimental and the simulated $\beta$ parameters are plotted as a function of the initial domains size. From that, by a direct comparison of the results, we can conclude that the domains range over $230 D^{0} A^{0}$ pairs for the highest laser intensity, in contrast to the lowest laser power 
where the domain size is only about $100 D^{0} A^{0}$. This difference is explained by the fact that at the vicinity of the phase transition temperature $T_{\mathrm{NI}}$ the potential energy surface reveals several separated, but energetically equal minima [24], as illustrated in Figure 32. Furthermore, we assume that at the beginning of the growth process, several domains can merge leading to large domains, also a coupling to neighboring stacks cannot be excluded. Additionally, for smaller photon flux, the initial domain is smaller since the number of merging processes is reduced.

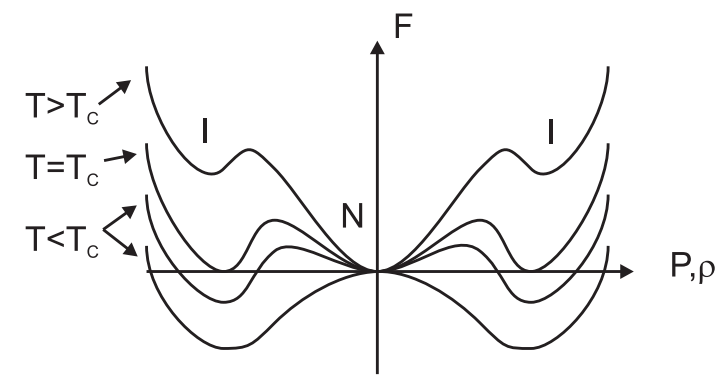

Figure 32. The free energy of a first-order transition is depicted as a function of the order parameter (for instance polarization, ionicity) for various temperatures at the vicinity of the phase transition. At the transition, several minima exist.

By cooling, the domain size is reduced continuously from $80 D^{0} A^{0}$ at $T=73 \mathrm{~K}$ to $20 D^{0} A^{0}$ for $68 \mathrm{~K}$. This can be explained in the picture of the potential energy surface, displayed in Figure 32. At lower temperatures the minimum of the ionic phase is below the energy minimum of the neutral phase and hence stable. Additionally, the weak local minimum of the neutral phase is only separated from the ionic phase by a small energy barrier which supports a very fast relaxation process [104]. Also, the possibility to end up in the neutral phase is reduced since the potential energy surface of the ground and excited state have changed their shape. By that, the route to reach the neutral state becomes much more difficult.

The simulation does not take into account some influencing parameters such as coupling to adjacent stacks, i.e., higher dimensions, or pinning centers, for example impurities. Also, the hopping rate was set to $50 \%$ for both directions along the stack which can deviate in reality. However, the theoretical calculations are a very good approach to describe the experimental behavior.

\section{Conclusions}

We reported our broad and extensive studies of the mixed-stacked organic charge-transfer salt TTF-CA that exhibits a neutral-ionic phase transition at $T_{\mathrm{NI}}=81.5 \mathrm{~K}$. The ionic phase is marked by a strong dimerization of the TTF and CA pairs and abrupt change of the molecular ionicity. Single crystals of TTF-CA were grown by the Stuttgart sublimation technique, the high quality was confirmed by $\mathrm{X}$-ray diffraction measurements and transport studies. Comprehensive theoretical calculations on the basis of DFT of the band structure and of the optical properties were performed and compared with experimental results, which yields very good agreement and reveals the same temperature dependence. Also, the gap feature is reproduced precisely and argues that the mid-infrared band consists of several transitions. Additionally, the resonance frequency and infrared intensity of vibrational modes were theoretically calculated and used to assign the vibrational features.

Extensive optical studies along the $a$ - and $b$-direction were conducted in order to examine the temperature-dependent evolution of the vibrational frequency. By taking a closer look, we could observe several modes, which exhibit a distinguishable jump at the neutral-ionic phase transition. The shift of the $v_{10}\left(\mathrm{~b}_{1 \mathrm{u}}\right)$ mode of the CA molecules was used to determine the temperature dependence of the ionicity.

Furthermore, by exciting the TTF-CA crystal with short laser pulses below $T_{\mathrm{NI}}$, a phase transition could be induced. The time-dependent reflectivity variation was probed in the complete mid-infrared 
spectral range using time-resolved Fourier-transform spectroscopy. The measured spectrum resembles the shape of the reflectivity difference $\Delta_{T} R$ between the spectrum above and below $T_{\mathrm{NI}}$. This implies that neutral, one-dimensional and metastable domains are created in the ionic phase. The observed characteristic time scale is between the microsecond and millisecond range. It was shown that the decay profile can be successfully fitted by a Kohlrausch-Williams-Watt function. A one-dimensional random-walk annihilation process of metastable neutral-ionic domain walls (NIDW), can explain the made observations very well. We can numerically simulate the process in all details and dependences.

By a comparison of the experimental and theoretical Kohlrausch-Williams-Watt parameter $\beta$, the domain size was estimated leading to the expansion of the established concept of the photo-induced domain size, as depicted in Figure 33. At low temperatures, the domain size is smaller, in general, and decreases when the laser intensity is reduced (see Figure 33c,d). By approaching the transition temperature $T_{\mathrm{NI}}$, the domain size is enlarged (see Figure 33a,b) due to the valance instability at the vicinity of the phase transition, as exemplary sketched in Figure 32.

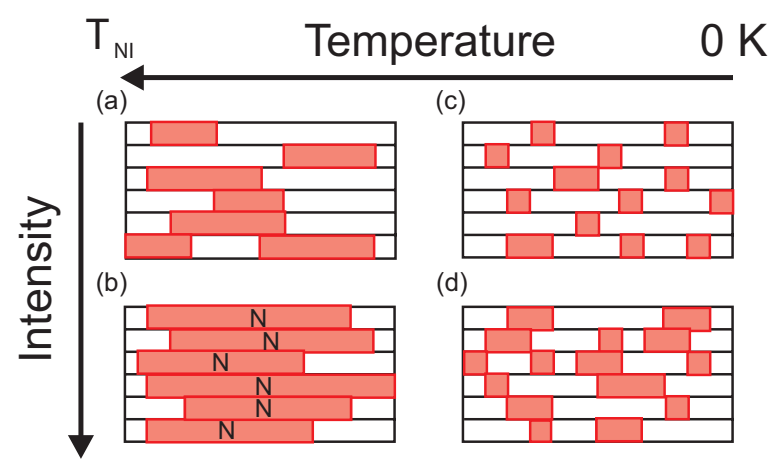

Figure 33. Size of the photo-generated domains at vicinity of $T_{\mathrm{NI}}$. (a) At low laser intensity, the domain size is small compared to (b) high laser power; (c) When temperature is reduced the domain size is smaller and (d) almost independent of the laser intensity.

However, the photo-induced creation of neutral-ionic domain walls can lead to a significant time-dependent photocurrent, which should be examined in future studies. In this context, the influence of electric fields on the domain walls can become interesting. Furthermore, photo-created spins can be detected by time-resolved electron-spin resonance experiments. This study should not be restricted to TTF-CA and should be extended to other mixed-stacked compounds.

Acknowledgments: Many valuable discussions with Alberto Girlando, Tomislav Ivek, and Eva Rose are acknowledged. We thank Gabriele Untereiner for the crystal growth and sample preparation. The project was supported by the Deutsche Forschungsgemeinschaft (DFG) and by the Carl-Zeiss-Stiftung. We would like to thank the bwGRiD [116] for providing the computational resources.

Author Contributions: M.D. and T.P. conceived and designed the experiments; T.P. performed the experiments and simulations, and analyzed the data; M.D. and T.P. wrote the paper.

Conflicts of Interest: The authors declare no conflict of interest.

\section{Appendix A. Light-Matter Interaction}

\section{Appendix A.1. Drude Model}

In solid-state materials, different kinds of electronic states can be excited depending on the electronic bands being empty, full, or partially occupied. Aside the electronic states, described by the electronic bands in bulk material, they exhibit vibrational states which can be excited, for instance, by infrared light. Based on the electronic transitions, generally one can distinguish between intra- and interband transitions. First of all, the intraband transitions will be treated. Especially in the case of a metal, they mainly contribute to the optical conductivity in the low-frequency range. Starting from 
the Drude model [98] describing a non-interacting electron gas, one can derive the following equation for damped charge carriers in an external electric field $\vec{E}(t)=\vec{E}_{0} e^{-i \omega t}$ :

$$
m_{\mathrm{e}} \frac{d \vec{r}^{2}}{d t^{2}}+\frac{m_{\mathrm{e}}}{\tau_{S c}} \frac{d \vec{r}}{d t}=-e \vec{E}(t)
$$

The second term on the left side describes the damping of the charge carrier motion due to elastic scattering at impurities, phonons or defects. The strength of the damping depends on the relaxation rate $\tau_{\mathrm{Sc}}$ which defines the elapsed time between two scattering events. After solving the differential equation, one receives the following expression for the complex optical conductivity function $\hat{\sigma}(\omega)=\sigma_{1}(\omega)+\mathrm{i} \sigma_{2}(\omega)$ :

$$
\hat{\sigma}(\omega)=\frac{N_{\mathrm{C}} e^{2} \tau_{S c}}{m_{\mathrm{e}}} \frac{1}{\left(1-i \omega \tau_{S c}\right)}=\frac{\sigma_{\mathrm{DC}}}{\left(1-i \omega \tau_{S c}\right)}
$$

with the charge carrier density $N_{C}$ and the charge carrier mass $m_{e}$. From Equation (A2), the real and imaginary conductivity, $\sigma_{1}(\omega)$ and $\sigma_{2}(\omega)$, can be easily derived:

$$
\sigma_{1}(\omega)=\frac{\omega_{p}^{2} \tau_{S c}}{4 \pi} \frac{1}{1+\omega^{2} \tau_{S c}^{2}} \quad \text { and } \quad \sigma_{2}(\omega)=\frac{\omega_{p}^{2} \tau_{S c}}{4 \pi} \frac{\omega \tau_{S c}}{1+\omega^{2} \tau_{S c}^{2}} .
$$

The parameter $\omega_{p}=\frac{N_{C} e^{2}}{m_{\mathrm{e}}}=\frac{\sigma_{\mathrm{DC}}}{\tau_{\mathrm{Sc}}}$ is the plasma frequency and can be used to calculate the number of free charge carriers if the mass $m_{e}$ is known. In this model, the quantum mechanical nature of the charge carriers has been neglected and solely treated classically. If one includes the quantum mechanical consideration of Sommerfeld, the electron-electron interaction and other interaction processes, the extended form of the Drude Equation (A2) is obtained in which the charge carrier mass $m_{e}$ and the scattering time $\tau_{\mathrm{Sc}}$ are replaced by a modified frequency-dependent effective charge carrier mass $m^{*}$ and scattering time $\tau_{\mathrm{Sc}^{\prime}}^{*}$, respectively [98].

\section{Appendix A.2. Drude-Lorentz Model}

In the case of interband transitions, an electron is excited by a photon from the occupied valence band into the unoccupied conduction band, while a hole is created in the valence band. This kind of transition appears, for instance, in systems with an energy gap, such as semiconductors or insulators; whose resonance energy is predominantly above the optical response of the free charge carriers. Here, the transition can occur directly or indirectly from the valence to the conduction band, but always under the premise that energy $E_{L}(\vec{k})+\hbar \omega=E_{V}\left(\vec{k}^{\prime}\right)$ and momentum $\vec{k} \pm \vec{q}_{\text {Phonon }}=\vec{k}^{\prime}$ are conserved. The optical response can be derived from a classic model of a damped harmonic oscillator. Thereby, a similar equation of motion as the Equation (A1) is used, but with the additional appended term $D \vec{r}$ describing the fundamental resonance frequency of the harmonic system.

$$
m_{\mathrm{e}} \frac{d \vec{r}^{2}}{d t^{2}}+\frac{m_{\mathrm{e}}}{\tau} \frac{d \vec{r}}{d t}+D \vec{r}=-e \vec{E}(t)
$$

After solving the differential equation and further transformations, the complex optical conductivity is

$$
\hat{\sigma}(\omega)=\frac{N e^{2} \tau}{m_{\mathrm{e}}} \frac{\omega}{\left(\omega+i\left(\omega_{0}^{2}-\omega^{2}\right) \tau\right)} .
$$

Thereby, omega $a_{0}=\sqrt{D / m_{\mathrm{e}}}$ is the resonance frequency of the undamped harmonic oscillator, depending on $m_{e}$ and on the restoring constant $D$, and the number of excited particles $N$. $\tau$ is the 
life time of the excited states and its reciprocal value corresponds to the line width of the resonance. Equation (A5) can be separated into an imaginary and real part:

$$
\sigma_{1}(\omega)=\frac{\omega_{p}^{2}}{4 \pi} \frac{\omega^{2}}{\tau\left(\omega_{0}^{2}+\omega^{2}\right)^{2}-\frac{\omega^{2}}{\tau}} \quad \text { and } \quad \sigma_{2}(\omega)=-\frac{\omega_{p}^{2}}{4 \pi} \frac{\omega\left(\omega_{0}^{2}-\omega^{2}\right)}{\left(\omega_{0}^{2}-\omega^{2}\right)^{2}-\frac{\omega^{2}}{\tau^{2}}}
$$

This model is not only valid for electronic excitations, but also for transitions between different vibrational levels.

\section{Appendix A.3. Fano Model}

In the early 1960s, Fano [117] presented his frequently cited theoretical work about the inelastic scattering process of electrons from the $1 s^{2}$ state in a helium atom into the $2 \mathrm{~s} 2 \mathrm{p}$ state. There, he described the excitation of the electron from the $s$-orbital into the $p$-orbital which resides in an energetic point of view in a continuum of undisturbed states. In the experimental excitation, asymmetric resonances have been observed unexpectedly. Fano [117] could now model this line shape by taking into account the interaction of the excited state with a continuum of states.

Such a coupling of discrete states of any kind to a continuum is not only observed by scattering experiments, but also in organic conductors, for instance, where molecular vibrations interact with the electronic background arising from broad electronic intra- and interband transitions. The results are the asymmetric Fano line shapes of the vibrational features that can be seen in the spectra of Section 4 . This specific case is referred to as emv-coupling, where symmetric molecule oscillations modify the HOMO of the molecule and, therefore, become infrared-active.

For simplicity, we assume that a second molecule of the same type is located parallel to the first one, forming a dimer; note that in general, it does not have to be the same type of molecule. Both molecules vibrate with the same symmetric mode but with a phase shift of $\pi$, thus the modification of the HOMOs is also out of phase. By this, an electric dipole moment is induced along the connecting line between both molecules. Because of the light mass of the electrons in comparison to the nucleus, the temporal change of the HOMOs takes place instantaneously together with molecular vibrations so that the dipole moment changes with the same frequency as the resonance frequency of the molecular mode. This correlates with the idea that a charge is transferred from one molecule to the other and back again. Due to the phase shift, this transition becomes infrared-active. Only gerade molecule modes can couple effectively to the HOMO: $g_{i}$ specifies the coupling strength between the HOMOs and the molecular vibration and can be calculated as follows:

$$
g=\frac{\partial E_{\mathrm{HOMO}}}{\partial Q_{i}}
$$

with the energy of the HOMOs $E_{\mathrm{HOMO}}$ and the generalized normal coordinate $Q_{i}$ of the symmetric vibration. In the case of TTF, TMTTF, BEDT-TTF and many other molecules, the gerade modes containing the inner $\mathrm{C}=\mathrm{C}$ double bonds modify the $\mathrm{HOMO}$ the strongest. This kind of excitation only occurs in relation with a broad electronic background whereas the vibrational features in the spectra cannot be modeled anymore by the Lorentz function. For that reason, the emv-coupled modes have to be fitted with the so-called Fano function $[117,118]$ containing several parameters:

$$
\sigma_{1}(\omega)=\sigma_{0} \frac{\gamma \omega\left[\gamma \omega\left(q^{2}-1\right)+2 q\left(\omega^{2}-\omega_{0}^{2}\right)\right]}{\left(\omega^{2}-\omega_{0}^{2}\right)^{2}+\gamma^{2} \omega^{2}} \quad \text { and } \quad \sigma_{2}(\omega)=\sigma_{0} \frac{\gamma \omega\left[\left(q^{2}-1\right)\left(\omega^{2}+\omega_{0}^{2}\right)-2 \gamma \omega\right]}{\left(\omega^{2}-\omega_{0}^{2}\right)^{2}+\gamma^{2} \omega^{2}} .
$$

$\gamma$ is the line width, $\sigma_{0}$ the amplitude, $\omega_{0}$ the resonance frequency and $q$ the coupling strength.

\section{Appendix B. Theoretical Calculations}

In order to better understand our experimental results, we carried out numerical simulations of the electronic and vibrational properties. To that end, we have conducted quantum chemical 
calculations that allow us to determine and understand the physical properties of the organic salts and compared to the experimental results. The calculations were performed on the level of density functional theory (DFT). The Quantum Espresso package (Version 4.3.2 and 5.1) [51] was used to evaluate the band structure and the optical functions. The determination of the normal modes of the organic molecules, their resonance frequency as well as their infrared intensity was calculated with the Gamess-US $[119,120]$ and Spartan 13 software packages.

\section{Appendix B.1. Band Structure and Optical Functions}

For the band structure calculations, only crystal structures were used, determined by X-ray, neutron- or muon-scattering experiments. Based on previous experimental results, such as transport measurements, the materials are treated as a metal or an insulator, respectively. To determine the physical properties, the total energy of the system has to be regarded:

$$
\begin{aligned}
\mathcal{H} & =-\frac{\hbar^{2}}{2 m_{e}} \sum_{i} \nabla_{i}^{2}-\sum_{i, I} \frac{Z_{I} e}{\left|\vec{r}_{i}-\vec{R}_{I}\right|}+\frac{1}{2} \sum_{i \neq j} \frac{e^{2}}{\left|\vec{r}_{i}-\vec{r}_{j}\right|}-\sum_{I} \frac{\hbar^{2}}{2 M_{I}} \nabla_{I}^{2}+\frac{1}{2} \sum_{I \neq J} \frac{Z_{I} Z_{J}}{\left|\vec{R}_{I}-\vec{R}_{J}\right|} \\
& =\mathcal{T}_{e}+\mathcal{V}_{\text {ext }}+\mathcal{V}_{\text {int }}+\mathcal{T}_{I}+\mathcal{V}_{I I} .
\end{aligned}
$$

The first term describes the kinetic energy of the electrons, the second one the Coulomb-interaction of the electrons with the positive charged nuclei and the third one the interaction of the electrons with each other. The last two components take into account the kinetic energy of the atomic cores and their interaction with each other. The cores are more than a factor of 1000 heavier than the electrons. Therefore, the kinetic energy of the nuclei can be neglected. Since the electrons move much faster than the atomic nuclei, the atomic core potential for them is frozen. On the other hand, the atomic cores move in an electronic potential since the electrons adapt themselves instantaneously to any new atomic nuclei configuration. This consideration is known as the Born-Oppenheimer approximation. The goal is to solve the Schrödinger equation

$$
i \hbar \frac{\partial \Psi(\vec{r}, t)}{\partial t}=\mathcal{H} \Psi(\vec{r}, t)
$$

and to find the minimum energy of the system. It is achieved by determining the eigenstates and eigenfunctions of the total Hamiltonian $\mathcal{H}$ by the variational principle.

At the moment, DFT is the standard technique to solve Hamiltonians $\mathcal{H}$ and thus, to calculate the physical quantities of a many-body system. In DFT, it is assumed that the ground state energy and each term in the Hamiltonian can be expressed by functionals of the electronic density $n(\vec{r})=n(\vec{r})_{\downarrow}+n(\vec{r})_{\uparrow}$ with the spin states $\sigma=\uparrow$ and $\downarrow$ instead of the wave function $\Psi(\vec{r})$ :

$$
\mathcal{E}[n]=\mathcal{T}_{e}[n]+\mathcal{V}_{\text {ext }}[n]+\mathcal{V}_{i n t}[n]+\mathcal{V}_{x c}[n]
$$

In this representation, the kinetic energy of the nuclei $T_{I}$ and their Coulomb interaction $V_{I I}$ are neglected. The last term is the so-called exchange-correlation term $V_{x c}$ containing all not respected interactions, for example self-interaction, correlations, etc. The main task in DFT is to find the accurate $V_{x c}$ which describes the system properly.

Appendix B.1.1. Functional

There are different approaches, classified by Jacob's ladder [121], which have five steps varying in accuracy:

- $\quad$ Level 1: the most famous $V_{x c}$ is the so-called local density approximation (LDA) or local spin density approximation (LSDA) functional. It is assumed that $V_{x c}$ corresponds to the known exchange-correlation energy of a homogeneous electron gas, which is directly related to the electron density. LDA describes metallic systems very well as they can be approximated by 
an undisturbed electron gas. However, it fails to model systems in which the electron density strongly varies in space, such as semiconductors or insulators.

- Level 2: a significant improvement of the precision and results is achieved by taking into account the spatial variation of the electron density. It can be considered as an additional correction parameter for LSDA. These functionals are summarized under the keyword: generalized gradient approximation (GGA). The most famous GGA functionals are B88 [122], BLYP [123], PW91 [124], and Perdew-Burke-Enzerhof (PBE) [52,125].

- Level 3: when higher derivatives of the electron density are included, they are called meta-GGA functionals.

- Level 4: furthermore, hybrid-GGA functionals are used to describe atoms as well as molecules with a much higher precision. Different exchange and correlation functionals are mixed and combined with each other depending on their coefficient. If they are determined by fitting experimental data, they are called semi-empirical functionals, or they have to satisfy certain predefined conditions. The most used representatives are B3LYP [126-128], and PBE1PBE [129] functionals. For more detail, it is referred to the relevant literature [130].

- Level 5: radom phase approximation (RPA).

In the framework of this work, only the PBE-functional (Level 2) was employed for the band structure and optical spectra calculations since the considered organic salts are strongly anisotropic and the electrons or holes are more localized in comparison to pure metals, such as copper. Van der Waals force play an important role in organic compounds, which is not described accurately by LDA, but better by a GGA functional. Nevertheless, this remains a future task to implement the van der Waals interaction into the upcoming functionals. In addition, GGA as PBE yields better estimations of the band gaps than LDA which generally underestimates it.

In contrast, the B3LYP- or the EDF2 [42] functionals (Level 4) were used for the normal mode analysis. They yield very good results for organic molecules and their results agree very well with experimental data [44,131-133]. Therefore, they are widely-used and tested.

\section{Appendix B.1.2. Basis Set}

The main task of all ab-initio methods is to solve the Schrödinger Equation (B2). To that end, one has to appropriate the wave function of the system, which can be a single atomic orbital, a molecular orbital or the periodic wave function of a bulk material. In the case of DFT, at the beginning of each calculation, the wave functions are constructed by a linear combination of predefined wave functions, where the precision depends on the chosen basis set and its size. A complete basis set would, in principle, be capable of describing the system entirely, but this would be related to an extremely large effort of computational time and capacity. Therefore, one has to find a compromise between precision and CPU time. Several approaches were developed over several decades:

- One possibility is to start with localized orbitals that can be divided in two subgroups: Slater-orbitals and Gauss-orbitals. Considering only the last ones, they are built of polynomial functions multiplied with Gaussian functions $\left(\exp \left\{r^{2}\right\}\right)$. The main advantage of this mathematical construction is that the matrix element, i.e., integrals, can be solved analytically and hence, computing time can be reduced. The precision can be increased by enlarging the size of the basis function. Furthermore, diffuse and polarization function can be added to the standard basis set because they describe molecular bonds and charged states of molecules much better.

The Gaussian or Pople basis sets are mainly used for calculations of isolated molecules or structures, because the wave functions decay with increasing distance to the atom or molecule and thus the electron density $n(\vec{r})$ is only calculated where also charge is present. In addition, a Gaussian basis set also describes the region close to the nucleus rather well. 
- Instead of approximating the wave functions by a linear combination of Gaussian functions, they can be constructed from the linear combinations of plane waves:

$$
\Psi_{\vec{k}, n}(\vec{r})=\frac{1}{\sqrt{N_{e f f}}} e^{i \vec{k} \vec{r}} \cdot \sum_{m} c_{n, m}(\vec{k}) \cdot e^{i \vec{G}_{m} \vec{r}} .
$$

This construction is perfectly suitable for the description of any periodic crystal structure. Typically, the valence electrons are responsible for the bindings and the core electrons can be regarded as an additional correction. Since close to the atomic cores the wave function oscillates very strongly, they have to be described by large wave vectors $\vec{k}$. According to Schrödinger's Equation (B2), the kinetic energy of the electrons $E_{K i n}$ is a quadratic function of the $\vec{k}$-vector. Therefore, the kinetic energy of the core electrons is very large. This leads to an extremely large number of wave functions, in general, and makes it necessary to define a cutoff energy of the electrons in consideration the required precision. Thus, to limit the number of wave functions, the pure atomic potential is approximated by a pseudo potential.

The minimum cutoff-energy must be identified in respect to the total energy of the system. In Figure B1, the total energy of the organic compound TTF-CA is plotted as a function of the wave function cutoff energy $E_{\text {cut }}$ for a cutoff energy of the electron density $E_{\text {cut }, \rho}$ of $250 \mathrm{Ry}$. For the convergence test, ultrasoft pseudo potentials were used (see for more details the following Appendix B.1.3). Above $E_{\mathrm{cut}}=25 \mathrm{Ry}$, the total energy converges and does not change significantly anymore. Therefore, $E_{\mathrm{cut}}=30 \mathrm{Ry}$ is a good value for the calculations. The cutoff energy of the electron density $E_{\text {cut }, \rho}$ should be by a factor of four larger than $E_{\text {cut }}$ for norm-conserving pseudo potential In the case of ultrasoft pseudo potential, $E_{\mathrm{cut}, \rho}$ must be between 8 and 12 times larger than $E_{\text {cut }}$.

Moreover, the number of plane waves $N_{G} \propto \Omega_{\text {cell }} \cdot E_{\text {cut }}^{3 / 2}$ depends on the maximal defined kinetic energy $\frac{1}{2} \vec{G}_{\text {max }}^{2}=\frac{1}{2}(\vec{k}+\vec{G})^{2} \leq E_{\text {cut }}$ and the volume of the unit cell $\Omega_{\text {cell }}$. Note, the volume of the plane waves in the reciprocal space is $V_{\max }=\frac{4 \pi}{3} G_{\max }^{3}$ at which the volume of a single plane wave is $V_{P W}=\frac{2 \pi}{\Omega_{\text {cell }}}$. Hence, the necessary number of wave functions increases with increasing the unit cell size and is much larger in contrast to the localized approach with Gaussian functions, for instance. However, plane waves can also be used for isolated molecules, but for this purpose a very large super cell has to be defined so that the plane waves decay very fast within the cell and do not interact with their mirror image of the neighboring cells.

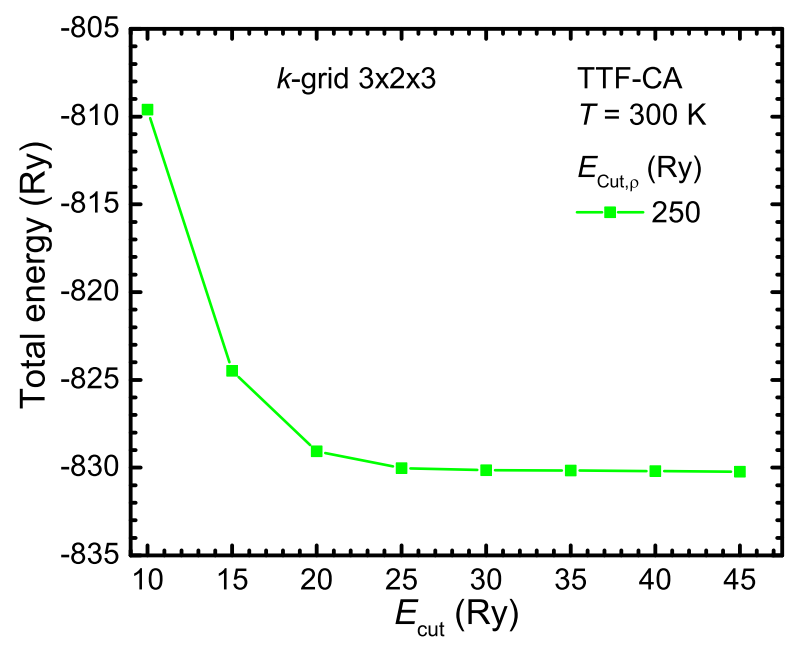

Figure B1. Total energy of TTF-CA at $300 \mathrm{~K}$ for a $3 \times 2 \times 3 k$-grid for an electron density cutoff energy of $E_{\mathrm{cut}, \rho}=250 \mathrm{Ry}$ as a function of $E_{\mathrm{cut}}$. Above $E_{\mathrm{cut}}=25 \mathrm{Ry}$, the total energy varies barely. 


\section{Appendix B.1.3. Pseudo Potential}

The number of plane waves can be substantially reduced by modeling the potential near the atomic core by a simple mathematical function. By that, the number of nodes and oscillations diminishes and hence, $\vec{k}$ and $E_{\text {cut }}$ decrease as well. The crucial point is that the valence electrons are modeled accurately since they are mainly responsible for the chemical bonds. The simplified function is called effective core potential or pseudo potential. To construct a pseudo potential, the full potential is split into two regions at a selected cutoff radius $r_{c}$, as depicted in Figure B2. For the calculation of a pseudo potential for an element of the periodic system, $r_{c}$ must be defined and afterwards tested.

There are two classes of pseudo potentials. The norm-conserving pseudo potential and wave functions form an orthonormal system. They have to satisfy four conditions:

1. The eigenvalues of the pseudo potential must agree with the ones of the real potential.

2. Above the cutoff radius $r_{c}$, the wave functions of the pseudo potential must be equal to the true total electron wave function.

3. The total charge within $r_{c}$ corresponds to the charge of the real total wave function.

4. At $r_{c}$ and $r_{c}>r$, the derivative of the pseudo-potential wave functions must agree with the real derivatives.

The second alternative, ultrasoft pseudo potential, which was developed by Vanderbilt [53], violates the third condition of the norm-conserving pseudo potential and has a larger $r_{c}$. There, the core potential for $r<r_{c}$ is weakened stronger so that the wave function oscillates less. Thus, the cutoff energy can be decreased and hence, the number of plane waves as well as the computing time are reduced. In the case of Quantum Espresso, they are limited to calculations of the band structure, density of states, and the total energy. Therefore, the optical spectra as well as the band structure are calculated solely by norm-conserving potentials.

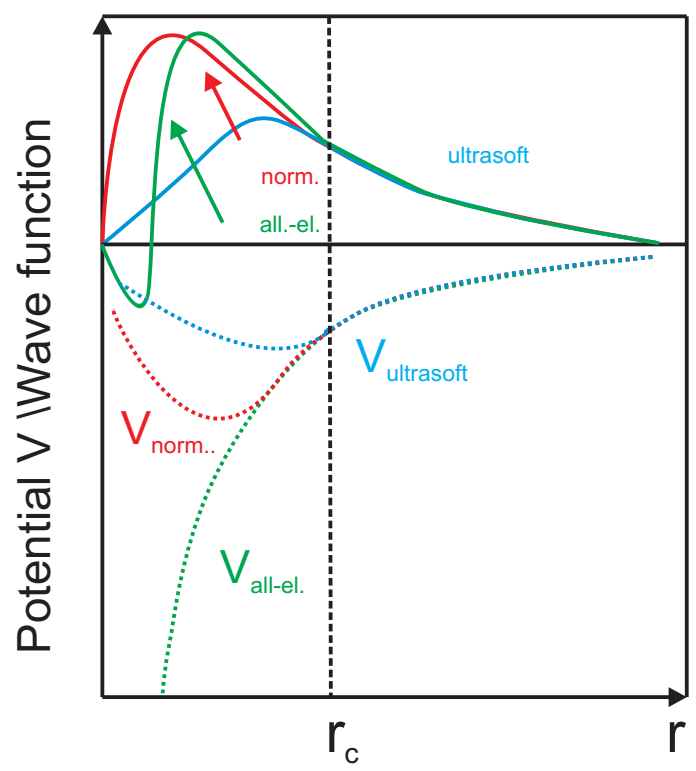

Figure B2. Exemplary comparison of the real wave function $\Psi_{\text {all.el. with the constructed norm-conserving }}$ and ultrasoft wave function $\Psi_{\text {norm. }}$ and $\Psi_{\text {ultrasoft }}$. The artificial wave function does not exhibit nodes and allows them to be simulated with a smaller number of plane waves, especially for the ultrasoft wave function. Above $r_{c}$, all wave functions show the same spatial evolution. Below $r_{c}$, the electron density $n(\vec{r})$ of $\Psi_{\text {ultrasoft }}$ differs from the true electron density, which must be renormalized. In contrast, $\Psi_{\text {norm. }}$ is considered to be norm conserving since its $n(\vec{r})$ is equal to electron density of $\Psi_{\text {all.el. }}$. To create the artificial wave function, the core potential has to be approximated by a corresponding $V_{\text {norm. }}$ or $V_{\text {ultrasoft }}$ potential being soften in the core region. This is actually the reason why the total wave function can be modeled by fewer plane waves. 
Appendix B.1.4. Band Structure

Before the band dispersion along a specific $k$-path can be determined, a self-consistent calculation has to be conducted on a dense, equally spaced $k$-grid within the Brillouin zone to obtain the exact wave functions of the system. This is necessary in order to guarantee that the derived band dispersions and energies are valid. For the calculation, we chose an automatic generated regular Monkhorst-grid [54] $a \times b \times c$. One can utilize the fact that the number of $k$-points scales reciprocally with the dimension of the unit cell. This allows the number of $k$-points for the long axis of an anisotropic unit cell to be diminished. The studied $k$-path was generated with visualization software Xcrysden [134].

Besides the convergence of the total energy with respect to the cutoff energy, its development as a function of grid size has to be checked as well. In Figure B3, such an evolution of the total energy of TTF-CA is studied as a function of the $k$-grid size. The total energy drops steeply and reveals no significant change above $3 \times 3 \times 3$. The calculation of the $k$-points was parallelized in Quantum Espresso and distributed between several cores. Afterwards, several post-processes have to be performed to receive a simple text file containing the information about the band structure.

Insulators and metals have to be treated differently within the calculations. In the case of insulators, the lowest bands $N=\frac{1}{2} N_{\text {elec }}$ are filled up to the band gap according to the Pauli-principle and the occupation is set to "fixed" in Quantum Espresso. However, it is important to add few empty bands in order to determine the wave function and the band gap accurately.

While, in principle, insulators are easy to handle, complications with metals occur because DFT-calculations are in general performed at $T=0 \mathrm{~K}$ and therefore, the bands are always filled up to the Fermi energy. This leads to a discontinuous jump of the wave function and their integrals, for instance the electron density, at the Fermi edge. Additionally, band-crossing can occur there during the calculations. Firstly, a larger number of $k$-points, as for insulators, are necessary to determine the dispersion of the bands at the vicinity of the Fermi energy precisely. Secondly, a finite temperature has to be introduced, by means of a smearing of the occupancy by a broadening function at the Fermi energy.

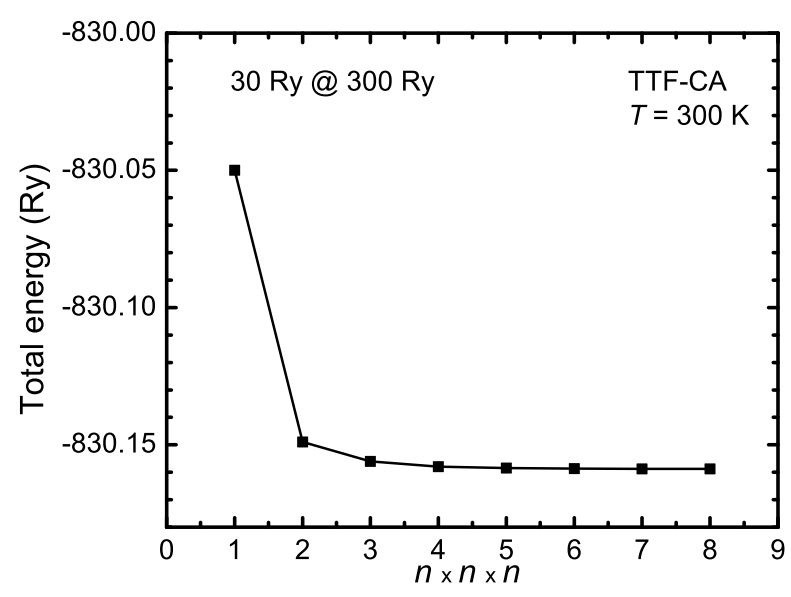

Figure B3. Illustration of the total energy of TTF-CA at $300 \mathrm{~K}$ as a function of $k$-grid size for $E_{\text {cut }}=30 \mathrm{Ry}$ and $E_{\mathrm{cut}, \rho}=300 \mathrm{Ry}$. The total energy is converged above a grid size of $3 \times 3 \times 3$. 
Appendix B.1.5. Dielectric Function

The Hamiltonian $\mathcal{H}$ of a system interacting with electromagnetic radiation consists of two parts:

$$
\mathcal{H}=\mathcal{H}_{0}+\mathcal{H}_{\text {int }}
$$

The first $\mathcal{H}_{0}$ is the unperturbed Hamiltonian and contains, for example, the electron-electron, the electron-ion and the electron-phonon interaction. The second term considers the interaction with an external vector potential $\vec{A}$ with the electrons:

$$
\mathcal{H}_{\text {int }} \propto \sum_{i}^{N}\left[\vec{p}_{i} \cdot \vec{A}\left(\vec{r}_{i}\right)-\vec{A}\left(\vec{r}_{i}\right) \cdot \vec{p}_{i}\right]
$$

at which a scalar potential field $\Phi$ is not considered as being responsible for the longitudinal response. Also, the quadratic term $\vec{A}^{2}(\vec{r})$ describing two-photon processes is neglected. $\mathcal{H}_{\text {int }}$ can be expressed by the electric current density $\vec{j}(\vec{r})[98]$ :

$$
\begin{gathered}
\mathcal{H}_{\text {int }} \propto \int \vec{j}(\vec{r}) \cdot \vec{A}(\vec{r}) d \vec{r} \\
\text { with } \quad \vec{j}(\vec{r}) \propto \sum_{i}^{N}\left[\vec{v}_{i} \delta\left(\vec{r}+\vec{r}_{i}\right)-\delta\left(\vec{r}-\vec{r}_{i}\right) \vec{v}_{i}\right] .
\end{gathered}
$$

The optical functions can be derived via Fermi's golden rule by the total absorbed power per volume $P \propto\left|\left\langle n^{*}\left|\mathcal{H}_{\text {int }}\right| n\right\rangle\right|^{2}=\omega \epsilon_{2} \vec{E}^{2}(\vec{r})=\sigma_{1} \vec{E}^{2}(\vec{r})$, in which the transverse electric field is connected to the vector field $\vec{A}(\vec{r})$ through the relation $\vec{E}(\vec{r})=i \frac{\omega}{c} \vec{A}(\vec{r})$.

The imaginary part of the dielectric tensor $\epsilon_{2, a, b}(\omega)$ can also be deduced from perturbation theory within the adiabatic and electric dipole approximation $A(\vec{r}) \propto \vec{E} \exp \{-i \vec{q} \cdot \vec{r}\} \propto \vec{E}$ as a response function $[98,135]$. Finally, after several transformations, the Drude-Lorentz function is derived:

$$
\begin{array}{r}
\epsilon_{2, a, b}(\omega)=\frac{4 \pi e^{2}}{\Omega N_{\vec{k}} m^{2}} \sum_{n, \vec{k}} \frac{\Delta \omega \hat{M}_{a, b}}{\omega^{4}+\Delta^{2} \omega^{2}}+\cdots+\sum_{n^{*}, n} \sum_{\vec{k}} \frac{\hat{M}_{a, b}}{E_{\vec{k}, n^{*}}-E_{\vec{k}, n}} \\
\cdots \cdots \frac{\Gamma \omega f\left(E_{\vec{k}, n}\right)}{\left[\left(\omega_{\vec{k}, n^{*}}-\omega_{\vec{k}, n}\right)^{2}-\omega^{2}\right]^{2}+\Gamma^{2} \omega^{2}}
\end{array}
$$

The real dielectric function $\epsilon_{1, a, b}$ is obtained by a Kramers-Kronig transformation. $\Gamma$ is the life time of the excited state, while $\Delta$ is the scattering time of the excited free charge carriers. The matrix transition element $\hat{M}_{a, b}$ is defined as follows:

$$
\begin{gathered}
\hat{M}_{a, b}=\left\langle\Psi_{\vec{k}, n^{*}}^{\star}\left|\hat{p}_{a}\right| \Psi_{\vec{k}, n}\right\rangle\left\langle\Psi_{\vec{k}, n}^{\star}\left|\hat{p}_{b}^{\dagger}\right| \Psi_{\vec{k}, n^{*}}\right\rangle \\
\hat{M}_{a, b}=\left\langle\Psi_{\vec{k}, n^{*}}^{\star}(\vec{r})\left|\frac{\partial}{\partial x_{a}}\right| \Psi_{\vec{k}, n}(\vec{r})\right\rangle\left\langle\Psi_{\vec{k}, n}^{\star}(\vec{r})\left|\frac{\partial}{\partial x_{b}}\right| \Psi_{\vec{k}, n^{*}}(\vec{r})\right\rangle .
\end{gathered}
$$

$\left|\Psi_{\vec{k}, n^{*}}\right\rangle$ is the Bloch wave function of the band $n^{*}$ being determined by the previous DFT calculation and $\hat{p}$ is the dipole transition operator. Only vertical transitions are taken into account with no change in $\vec{k}$. 
The complex dielectric function $\hat{\epsilon}(\omega)$ was calculated by the subroutine epsilon.x of Quantum Espresso. Prior to this, a self-consistent calculation of the total energy has to be performed and after all $\hat{\epsilon}(\omega)$ is ascertained by the determined eigenvalues and eigenfunctions. It is important that a dense grid of $k$-points is set to guarantee that all transitions are equally included.

\section{Appendix B.2. Normal Mode Analysis}

Lattice and molecular vibrations can be determined by the variation of the total energy in the thermodynamic equilibrium. Since the nuclei are much heavier than the light electrons, they move slower so that the contribution of the electrons can be neglected. Therefore, the total energy $E(\vec{R})$ of the system can be regarded as a function of the atomic nucleus coordinates $\vec{R} \equiv\left\{\vec{R}_{I}\right\}$ being called the potential-energy surface. The equation of motion for the nuclei I is:

$$
M_{I} \frac{\partial^{2} u_{I}(t)}{\partial t^{2}}=-\frac{\partial}{\partial \vec{R}_{I}} E(\vec{R})
$$

leading to a system of equations of coupled harmonic oscillators. $E(\vec{R})$ can be developed as a Taylor series at the global minimum of the potential-energy surface. The first-order term is zero due to the minimum of the total energy at the equilibrium and only the second-order term is considered:

$$
C_{I, a ; J, b}=\frac{\partial^{2} E(\vec{R})}{\partial R_{I, a} \partial R_{J, b}}
$$

$C_{I, a ; j, b}$ corresponds to the force constant. The equation of motion (B12) can now be expressed as

$$
\begin{aligned}
& -\omega^{2} M_{I} u_{I}(t)=-\sum_{J, b} C_{I, a ; J, b} u_{J, b} \\
& \operatorname{det}\left|\frac{1}{\sqrt{M_{I} M_{J}}} C_{I, a ; J, b}-\omega^{2}\right|=0
\end{aligned}
$$

with the displacement $u_{I, \alpha}(t)=u_{I, 0, \alpha} e^{i \omega t}$ of the nucleus $I$.

For the normal mode analysis, the molecular structures were drawn by chemical drawing software (i.e., Gabedit, Avogadro) or taken from existing structural data sets derived by scattering experiments. In general, the B3LYP [126] or the EPF2 [42]-functional were used in connection with a Gaussian basis set. To get the normal modes, it is necessary to optimize the structure by finding the global minimum of the potential-energy surface of the molecule. To this end, the coordinates of the atoms are relaxed and the gradient of the total energy determined, which has to be zero. Furthermore, the second derivative, the Hesse matrix $\mathbf{H}$, must be positive in all elements. It is assumed that in first approximation the potential-energy surface is quadratic for small displacements. 


\section{Appendix C. Vibrational Features of TTF and CA Molecules}

Table C1. Calculated frequencies in $\mathrm{cm}^{-1}$ and infrared intensities (Int.) given in units of $\mathrm{D}^{2} \mathrm{amu}^{-1} \cdot \AA^{-2}$ of neutral CA and ionized $\mathrm{CA}^{-}$.

\begin{tabular}{|c|c|c|c|c|c|c|c|c|}
\hline \multirow{2}{*}{ Label } & \multirow{2}{*}{ Symmetry } & \multicolumn{3}{|c|}{$\mathrm{CA}^{0}$} & \multicolumn{3}{|c|}{$\mathrm{CA}^{-}$} & \multirow{2}{*}{$\Delta v$} \\
\hline & & $v_{\text {calc }}$ & $v_{\text {scaled }}$ & Int. & $v_{\text {calc }}$ & $v_{\text {scaled }}$ & Int. & \\
\hline$v_{1}$ & \multirow{6}{*}{$a_{g}$} & 1754.1 & 1696.22 & - & 1548.51 & 1497.41 & - & -199 \\
\hline$v_{2}$ & & 1630.2 & 1576.24 & - & 1608.16 & 1555.09 & - & -21 \\
\hline$v_{3}$ & & 970.94 & 990.07 & - & 982.53 & 1002 & - & 11.82 \\
\hline$v_{4}$ & & 486.36 & 495.94 & - & 496.35 & 506.13 & - & 10.18 \\
\hline$v_{5}$ & & 317.71 & 323.97 & - & 320.91 & 327.23 & - & 3.26 \\
\hline$v_{6}$ & & 200.04 & 203.98 & - & 201.04 & 205 & - & 1.02 \\
\hline$v_{9}$ & $b_{1 g}$ & 322.03 & 328.37 & - & 328.8 & 335.3 & - & 6.92 \\
\hline$v_{15}$ & \multirow{3}{*}{$b_{2 g}$} & 801.56 & 817.35 & - & 771.93 & 787.13 & - & -29.93 \\
\hline$v_{16}$ & & 441.79 & 450.49 & - & 390.53 & 398.22 & - & -52.27 \\
\hline$v_{17}$ & & 96.76 & 98.66 & - & 120.16 & 119.02 & - & 21.5 \\
\hline$v_{23}$ & \multirow{5}{*}{$b_{3 g}$} & 1215.35 & 1175.25 & - & 1301.89 & 1258.92 & - & 83.68 \\
\hline$v_{24}$ & & 829 & 845.33 & - & 807.37 & 823.28 & - & -22.06 \\
\hline$v_{25}$ & & 733.82 & 748.28 & - & 724.12 & 738.4 & - & -10 \\
\hline$v_{26}$ & & 337.27 & 343.92 & - & 325.54 & 331.96 & - & -12 \\
\hline$v_{27}$ & & 263.81 & 269 & - & 275.46 & 281 & - & 11.88 \\
\hline$v_{7}$ & \multirow[b]{2}{*}{$a_{u}$} & 573.15 & 584.44 & - & 559.45 & 570.47 & - & -13.96 \\
\hline$v_{8}$ & & 69.76 & 71.13 & - & 75.07 & 76.54 & - & 5.36 \\
\hline$v_{10}$ & \multirow{5}{*}{$\mathrm{b}_{1 \mathrm{u}}$} & 1757 & 1699 & 349.46 & 1565.35 & 1513.7 & 288.1 & -185.31 \\
\hline$v_{11}$ & & 1086.54 & 1050.68 & 415.77 & 1117.9 & 1081 & 203.72 & 30.32 \\
\hline$v_{12}$ & & 899.07 & 916.78 & 25.31 & 892.53 & 910.12 & 159.74 & -6.67 \\
\hline$v_{13}$ & & 460.13 & 469.2 & 5.27 & 436.92 & 445.53 & 0.66 & -23.66 \\
\hline$v_{14}$ & & 205.26 & 209.3 & 0.03 & 205.46 & 209.51 & 1.44 & 0.21 \\
\hline$v_{18}$ & \multirow{5}{*}{$\mathrm{b}_{2 \mathrm{u}}$} & 1590.76 & 1538.26 & 254.54 & 1465 & 1416.57 & 0.27 & -121.7 \\
\hline$v_{19}$ & & 1202.63 & 1162.94 & 120.02 & 1099.8 & 1063.5 & 97.81 & -99.44 \\
\hline$v_{20}$ & & 723.56 & 737.81 & 206.15 & 686.72 & 700.25 & 155.1 & -37.56 \\
\hline$v_{21}$ & & 380.92 & 388.42 & 3.11 & 360.35 & 367.45 & 0.16 & -20.97 \\
\hline$v_{22}$ & & 214.24 & 218.46 & 0.23 & 213.82 & 218.04 & 0.73 & -0.43 \\
\hline$v_{28}$ & \multirow{3}{*}{$b_{3 u}$} & 756.16 & 771.05 & 27.3 & 732.12 & 746.54 & 17.92 & -24.51 \\
\hline$v_{29}$ & & 199.7 & 203.62 & 2.88 & 201.47 & 205.44 & 2.76 & 1.82 \\
\hline$v_{30}$ & & 69 & 70.33 & 1.68 & 86.8 & 88.5 & 3.12 & 18.17 \\
\hline
\end{tabular}


Table C2. Calculated frequencies $\left(\mathrm{cm}^{-1}\right)$ and infrared intensities $\left(\mathrm{D}^{2} \mathrm{amu} \mathrm{u}^{-1} \cdot \AA^{-2}\right)$ of neutral TTF and inonized $\mathrm{TTF}^{+}$.

\begin{tabular}{|c|c|c|c|c|c|c|c|c|}
\hline \multirow{2}{*}{ Label } & \multirow{2}{*}{ Symmetry } & \multicolumn{3}{|c|}{$\mathrm{TTF}^{0}$} & \multicolumn{3}{|c|}{$\mathrm{TTF}^{+}$} & \multirow{2}{*}{$\Delta v$} \\
\hline & & $v_{\text {calc }}$ & $v_{\text {scaled }}$ & Int. & $v_{\text {calc }}$ & $v_{\text {scaled }}$ & Int. & \\
\hline$v_{1}$ & \multirow{7}{*}{$a_{g}$} & 3226.47 & 3120 & - & 3236.63 & 3129.82 & - & 9.83 \\
\hline$v_{2}$ & & 1621.92 & 1568.39 & - & 1551.15 & 1499.96 & - & -68.43 \\
\hline$v_{3}$ & & 1.576 & 1524.41 & - & 1427.75 & 1380.63 & - & -143.78 \\
\hline$v_{4}$ & & 1.125 & 1088 & - & 1130 & 1092.71 & - & 4.72 \\
\hline$v_{5}$ & & 722.50 & 736.73 & - & 737.72 & 752.25 & - & 15.52 \\
\hline$v_{6}$ & & 466.1 & 475.28 & - & 501.95 & 511.84 & - & 36.56 \\
\hline$v_{7}$ & & 248.6 & 253.5 & - & 262.21 & 267.38 & - & 13.88 \\
\hline$v_{11}$ & \multirow{2}{*}{$b_{1 g}$} & 849 & 865.71 & - & 875.55 & 892.8 & - & 27.1 \\
\hline$v_{12}$ & & 419.08 & 427.34 & - & 435.66 & 444.24 & - & 16.91 \\
\hline$v_{19}$ & \multirow{3}{*}{$b_{2 g}$} & 632.63 & 645.09 & - & 688.56 & 702.13 & - & 57.03 \\
\hline$v_{20}$ & & 498.9 & 508.73 & - & 513.54 & 523.65 & - & 14.92 \\
\hline$v_{21}$ & & 93.78 & 95.63 & - & 154.6 & 157.6 & - & 61.96 \\
\hline$v_{28}$ & \multirow{6}{*}{$b_{3 g}$} & 3206 & 3100.2 & - & 3220.42 & 3114.15 & - & 14 \\
\hline$v_{29}$ & & 1289.96 & 1247.4 & - & 1298.58 & 1255.72 & - & 8.34 \\
\hline$v_{30}$ & & 967.9 & 986.97 & - & 1021.58 & 987.87 & - & 0.9 \\
\hline$v_{31}$ & & 796.98 & 812.68 & - & 824.12 & 840.36 & - & 27.68 \\
\hline$v_{32}$ & & 612.32 & 624.38 & - & 627.6 & 639.96 & & 15.56 \\
\hline$v_{33}$ & & 305.94 & 311.97 & - & 301.15 & 307.08 & - & -4.88 \\
\hline$v_{8}$ & \multirow{3}{*}{$a_{u}$} & 848.96 & 865.68 & - & 873.54 & 890.74 & - & 25.06 \\
\hline$v_{9}$ & & 415.72 & 423.91 & - & 424.94 & 433.31 & - & 9.40 \\
\hline$v_{10}$ & & 92.4 & 94.22 & - & 65.36 & 66.64 & - & -27.57 \\
\hline$v_{13}$ & \multirow{6}{*}{$\mathrm{b}_{1 \mathrm{u}}$} & 3226.5 & 3120.03 & 0.5 & 3236.64 & 3129.83 & 28.57 & 9.8 \\
\hline$v_{14}$ & & 1598.57 & 1545.82 & 23.07 & 1532 & 1481.43 & 111.48 & -64.38 \\
\hline$v_{15}$ & & 1124.65 & 1087.54 & 3.15 & 1130.92 & 1094 & 0.12 & 6.06 \\
\hline$v_{16}$ & & 764.21 & 779.27 & 26.39 & 812 & 828 & 36.55 & 48.72 \\
\hline$v_{17}$ & & 720.14 & 734.33 & 9.13 & 726.8 & 741.15 & 7.63 & 6.82 \\
\hline$v_{18}$ & & 434.26 & 442.82 & 20.44 & 468.17 & 477.4 & 14.72 & 34.58 \\
\hline$v_{22}$ & \multirow{6}{*}{$\mathrm{b}_{2 \mathrm{u}}$} & 3206.9 & 3101.04 & 4.1 & 3220.6 & 3114.31 & 30.46 & 13.27 \\
\hline$v_{23}$ & & 1287.6 & 1245.11 & 0.02 & 1294.56 & 1251.84 & 8 & 6.73 \\
\hline$v_{24}$ & & 823.4 & 839.62 & 7.7 & 868.33 & 885.44 & 7 & 45.82 \\
\hline$v_{25}$ & & 786.91 & 802.41 & 56 & 823.81 & 840.04 & 26.51 & 37.63 \\
\hline$v_{26}$ & & 621.37 & 633.61 & 2.76 & 636.68 & 649.27 & 1.19 & 15.61 \\
\hline$v_{27}$ & & 114.31 & 116.56 & 0.58 & 123.45 & 125.88 & 0.28 & 9.32 \\
\hline$v_{34}$ & \multirow{3}{*}{$b_{3 u}$} & 633 & 645.46 & 160.31 & 690.13 & 703.73 & 164.7 & 58.27 \\
\hline$v_{35}$ & & 243.12 & 247.91 & 1.64 & 332.33 & 338.87 & 3.93 & 90.97 \\
\hline$v_{36}$ & & 51.44 & 52.45 & 3.69 & 101.27 & 103.27 & 4.85 & 50.81 \\
\hline
\end{tabular}

\section{Appendix D. Heating Effect by Laser Radiation}

The starting point is the inhomogeneous heat conduction equation:

$$
\vec{\nabla}^{2} T(\vec{r}, t)-\frac{\rho_{m} C_{\mathrm{p}}}{\lambda_{\text {therm }}} \frac{\partial T(r, t)}{\partial t}=-\frac{Q_{\mathrm{p}}(1-R) \alpha e^{-\alpha z} f(r) q(t)}{\lambda_{\text {therm }}} .
$$

On the left side of the equation, the first term is the spatial derivative of temperature describing the spatial heat flux, the second term reflects the time-dependent change of temperature, which is normalized to the thermal diffusivity $\kappa=\frac{\lambda_{\text {therm }}}{\rho_{m} C_{\mathrm{p}}}$. The right side considers the heat input due to a laser pulse with the temporal and spatial profile $q(t)$ and $f(r)$. In addition, the equation contains further intrinsic material parameters: the reflectivity $R(v)$, the irradiance $Q_{p}$ and the absorption coefficient $\alpha$. For $t>\tau_{\mathrm{p}}$, a laser pulse with a constant pulse length of $\tau_{p}=8 \mathrm{~ns}$ and a spatial uniform profile with 
a diameter of $d=600 \mu \mathrm{m}$ was assumed to determine the following dependence of temperature with distance $z$ from the surface as a function of time $t$ by solving Equation (D1):

$$
\Delta T(z, t)=\frac{2 Q_{\mathrm{p}}(1-R(v))}{\lambda_{\text {therm }}} \sqrt{\left(\kappa \tau_{\mathrm{p}}\right)}\left[\sqrt{\left(t \tau_{\mathrm{p}}\right)} \operatorname{ierfc}\left\{\frac{z}{2 \sqrt{\kappa t}}\right\}-\sqrt{\left(t \tau_{\mathrm{p}}^{-1}-1\right)} \operatorname{ierfc}\left\{\frac{z}{2 \sqrt{\kappa\left(t-\tau_{\mathrm{p}}\right)}}\right\}\right]
$$

with

$$
\operatorname{ierfc}\{x\}=\int_{x}^{\infty} \operatorname{erfc}\{y\} \mathrm{d} y
$$

for $z=0$ the equation arises from Equation (9) which was derived from Equation (D1)

$$
\Delta T(t)=\frac{2 Q_{\mathrm{p}}(1-R)}{\lambda} \frac{\left(\kappa \tau_{\mathrm{p}}\right)^{0.5}}{\pi^{0.5}}\left(\left(t \tau_{\mathrm{p}}^{-1}\right)^{0.5}-\left(t \tau_{\mathrm{p}}^{-1}-1\right)^{0.5}\right)
$$

for $\tau_{\mathrm{p}}>t$. The temporal temperature profile for TTF-CA, derived from Equation (D2), is plotted in Figure D1 for different positions $z$ inside the sample. The used parameters correspond to the values in Section 5.4. With increasing distance from the sample surface, the maximum of the temperature change shifts to later points in time. At a depth of $500 \mathrm{~nm}$, the maximum of the temperature change is located at $50 \mathrm{~ns}$ and resides below the transition temperature of $81.5 \mathrm{~K}$. In a distance of $100 \mathrm{~nm}$, the temperature is above $T_{\mathrm{NI}}$, but drops sharply below it after $100 \mathrm{~ns}$.

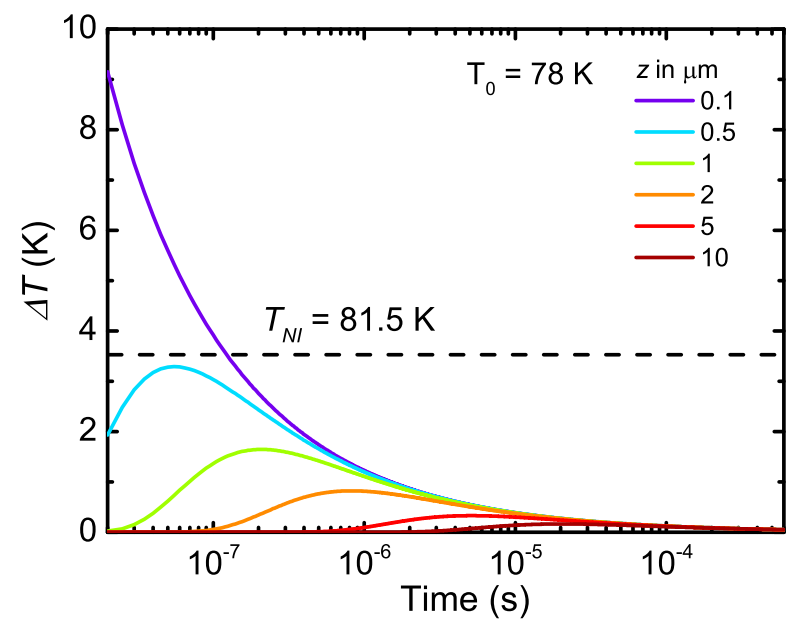

Figure D1. Temporal evolution of the change of sample temperature of TTF-CA for a laser intensity of $0.71 \mathrm{~mJ} / \mathrm{cm}^{2}$ at $T_{0}=78 \mathrm{~K}$ derived from Equation (D2). Starting from a depth of $500 \mathrm{~nm}$, the maximum of the temperature profile stays clearly below $T_{\mathrm{NI}}=81.5 \mathrm{~K}$.

\section{References}

1. Torrance, J.B.; Mayerle, J.J.; Lee, V.Y.; Bechgaard, K. A new class of highly conducting organic materials: Charge-transfer salts of the tetrathiafulvalenes with the tetrahalo- $p$-benzoquinones. J. Am. Chem. Soc. 1979, 101, 4747-4748.

2. Le Cointe, M.; Lemée-Cailleau, M.H.; Cailleau, H.; Toudic, B.; Toupet, L.; Heger, G.; Moussa, F.; Schweiss, P.; Kraft, K.H.; Karl, N. Symmetry breaking and structural changes at the neutral-to-ionic transition in tetrathiafulvalene-p-chloranil. Phys. Rev. B 1995, 51, 3374-3386.

3. Mayerle, J.J.; Torrance, J.B.; Crowley, J.I. Mixed-stack complexes of tetrathiafulvalene. The structures of the charge-transfer complexes of TTF with chloranil and fluoranil. Acta Cryst. B 1979, 35, 2988-2995.

4. Girlando, A.; Marzola, F.; Pecile, C.; Torrance, J.B. Vibrational spectroscopy of mixed stack organic semiconductors: Neutral and ionic phases of tetrathiafulvalene-chloranil (TTF-CA) charge transfer complex. J. Chem. Phys. 1983, 79, 1075-1085.

5. Jacobsen, C.S.; Torrance, J.B. Behavior of charge-transfer absorption upon passing through the neutral-ionic phase transition. J. Chem. Phys. 1983, 78, 112-115. 
6. Le Cointe-Buron, M.; Lemée-Cailleau, M.H.; Cailleau, H.; Luty, T. Condensation of Self-Trapped Charge-Transfer Excitations and Interplay between Quantum and Thermal Effects at the Neutral-to-Ionic Transition. J. Low Temp. Phys. 1998, 111, 677-691.

7. Cointe, M.L.; Lemée-Cailleau, M.; Cailleau, H.; Toudic, B. Structural aspects of the neutral-to-ionic transition in mixed-stack charge-transfer complexes. J. Mol. Struct. 1996, 374, 147-153.

8. Girlando, A.; Bozio, R.; Pecile, C.; Torrance, J.B. Discovery of vibronic effects in the Raman spectra of mixed-stack charge-transfer crystals. Phys. Rev. B 1982, 26, 2306-2309.

9. Okamoto, H.; Mitani, T.; Tokura, Y.; Koshihara, S.; Komatsu, T.; Iwasa, Y.; Koda, T.; Saito, G. Anomalous dielectric response in tetrathiafulvalene- $p$-chloranil as observed in temperature- and pressure-induced neutral-to-ionic phase transition. Phys. Rev. B 1991, 43, 8224-8232.

10. Ayache, C.; Torrance, J. Multiple specific heat anomalies at the neutral-ionic phase transition in TTF-chloranil. Solid State Commun. 1983, 47, 789-793.

11. Mitani, T.; Kaneko, Y.; Tanuma, S.; Tokura, Y.; Koda, T.; Saito, G. Electric conductivity and phase diagram of a mixed-stack charge-transfer crystal: Tetrathiafulvalene-p-chloranil. Phys. Rev. B 1987, 35, 427-429.

12. Tokura, Y.; Okamoto, H.; Koda, T.; Mitani, T.; Saito, G. Nonlinear electric transport and switching phenomenon in the mixed-stack charge-transfer crystal tetrathiafulvalene-p-chloranil. Phys. Rev. B 1988, 38, 2215-2218.

13. McConnell, H.M.; Hoffman, B.M.; Metzger, R.M. Charge Transfer in Molecular Crystals. Proc. Natl. Acad. Sci. USA 1965, 53, 46-50.

14. Torrance, J.B.; Vazquez, J.E.; Mayerle, J.J.; Lee, V.Y. Discovery of a Neutral-to-Ionic Phase Transition in Organic Materials. Phys. Rev. Lett. 1981, 46, 253-257.

15. Katan, C. First-Principles Study of the Structures and Vibrational Frequencies for Tetrathiafulvalene TTF and TTF- $d_{4}$ in Different Oxidation States. J. Phys. Chem. A 1999, 103, 1407-1413.

16. Lichtenberger, D.L.; Johnston, R.L.; Hinkelmann, K.; Suzuki, T.; Wudl, F. Relative electron donor strengths of tetrathiafulvene derivatives: Effects of chemical substitutions and the molecular environment from a combined photoelectron and electrochemical study. J. Am. Chem. Soc. 1990, 112, 3302-3307.

17. Sato, N.; Inokuchi, H.; Shirotani, I. Polarization energies of tetrathiafulvalene derivatives. Chem. Phys. 1981, 60, 327-333.

18. Batsanov, A.S.; Bryce, M.R.; Heaton, J.N.; Moore, A.J.; Skabara, P.J.; Howard, J.A.K.; Orti, E.; Viruela, P.M.; Viruela, R. New functionalized tetrathiafulvalenes: $\mathrm{X}$-ray crystal structures and physico-chemical properties of TTF-C(O)NMe $\mathrm{NM}_{2}$ and TTF-C(O)-O- $\mathrm{C}_{4} \mathrm{H}_{9}$ : A joint experimental and theoretical study. J. Mater. Chem. 1995, 5, 1689-1696.

19. Cooper, C.D.; Frey, W.F.; Compton, R.N. Negative ion properties of fluoranil, chloranil, and bromanil: Electron affinities. J. Chem. Phys. 1978, 69, 2367-2374.

20. Tanaka, S.; Aoki, S.; Nakayama, T.; Egusa, S. Effects of electrostatic field on the phase properties of mixed-stack organic charge-transfer compounds. Phys. Rev. B 1995, 52, 1549-1565.

21. Dengl, A.; Beyer, R.; Peterseim, T.; Ivek, T.; Untereiner, G.; Dressel, M. Evolution of ferroelectricity in tetrathiafulvalene- $p$-chloranil as a function of pressure and temperature. J. Chem. Phys. 2014, 140, 244511.

22. Nagaosa, N.; Takimoto, J.I. Theory of Neutral-Ionic Transition in Organic Crystals. I. Monte Carlo Simulation of Modified Hubbard Model. J. Phys. Soc. Jpn. 1986, 55, 2735-2744.

23. Nagaosa, N.; Takimoto, J.I. Theory of Neutral-Ionic Transition in Organic Crystals. II. Effect of the Intersite Coulomb Interaction. J. Phys. Soc. Jpn. 1986, 55, 2745-2753.

24. Soos, Z.G.; Painelli, A. Metastable domains and potential energy surfaces in organic charge-transfer salts with neutral-ionic phase transitions. Phys. Rev. B 2007, 75, 155119.

25. Mitani, T.; Tokura, Y.; Kaneko, Y.; Takaoka, K.; Koda, T.; Saito, G. Phase transition of the mixed-stack charge-transfer TTF-p-chloranil single crystals. Synth. Met. 1987, 19, 515-520.

26. Kambe, T.; Nogami, Y.; Oshima, K.; Takahashi, Y.; Sakai, H.; Koshihara, S. Soliton dynamics in TTF-CA. Synth. Met. 1999, 103, 1824.

27. Mitani, T.; Saito, G.; Tokura, Y.; Koda, T. Soliton Formation at the Neutral-to-Ionic Phase Transition in the Mixed-Stack Charge-Transfer Crystal Tetrathiafulvalene- $p$-Chloranil. Phys. Rev. Lett. 1984, 53, 842-845.

28. Takaoka, K.; Kaneko, Y.; Okamoto, H.; Tokura, Y.; Koda, T.; Mitani, T.; Saito, G. Infrared molecular-vibration spectra of tetrathiafulvalene-chloranil crystal at low temperature and high pressure. Phys. Rev. B 1987, $36,3884-3887$. 
29. Okamoto, H.; Koda, T.; Tokura, Y.; Mitani, T.; Saito, G. Pressure-induced neutral-to-ionic phase transition in organic charge-transfer crystals of tetrathiafulvalene-p-benzoquinone derivatives. Phys. Rev. B 1989, 39, 10693-10701.

30. Kaneko, Y.; Tanuma, S.; Tokura, Y.; Koda, T.; Mitani, T.; Saito, G. Optical reflectivity spectra of the mixed-stack organic charge-transfer crystal tetrathiafulvalene- $p$-chloranil under hydrostatic pressure. Phys. Rev. B 1987, 35, 8024-8029.

31. Tokura, Y.; Okamoto, H.; Koda, T.; Mitani, T. Pressure-induced neutral-to-ionic phase transition in TTF- $p$-chloranil studied by infrared vibrational spectrocopy. Solid State Commun. 1986, 57, 607-610.

32. Girlando, A.; Painelli, A. Regular-dimerized stack and neutral-ionic interfaces in mixed-stack organic charge-transfer crystals. Phys. Rev. B 1986, 34, 2131-2139.

33. Masino, M.; Girlando, A.; Brillante, A. Intermediate regime in pressure-induced neutral-ionic transition in tetrathiafulvalene-chloranil. Phys. Rev. B 2007, 76, 064114.

34. Lemée-Cailleau, M.H.; Le Cointe, M.; Cailleau, H.; Luty, T.; Moussa, F.; Roos, J.; Brinkmann, D.; Toudic, B.; Ayache, C.; Karl, N. Thermodynamics of the Neutral-to-Ionic Transition as Condensation and Crystallization of Charge-Transfer Excitations. Phys. Rev. Lett. 1997, 79, 1690-1693.

35. Luty, T.; Cailleau, H.; Koshihara, S.; Collet, E.; Takesada, M.; Lemée-Cailleau, M.H.; Cointe, M.B.L.; Nagaosa, N.; Tokura, Y.; Zienkiewicz, E.; et al. Static and dynamic order of cooperative multi-electron transfer. Europhys. Lett. 2002, 59, 619-625.

36. Engler, E.M.; Patel, V.V.; Andersen, J.R.; Tomkiewicz, Y.; Craven, R.A.; Scott, B.A.; Etemad, S. Synthetic Approaches to the Study of Organic Metals. Ann. N. Y. Acad. Sci. 1978, 313, 343-354.

37. Demaniets, L.N. Organic Crystals; Germanates; Semiconductors; Springer: Berlin/Heidelberg, Germany; New York, NY, USA, 1980.

38. Schwoerer, M.; Wolf, H. Organic Molecular Solids; Wiley-VCH: Weinheim, Germany, 2007.

39. Williams, J.M.; Ferraro, J.R.; Thorn, R.J.; Carlson, K.D.; Geiser, U.; Wang, H.H.; Kini, A.M.; Whangbo, M.H. Organic Superconductors (Including Fullerenes: Synthesis, Structure, Properties, and Theory); Prentice Hall: Englewood Cliffs, NJ, USA, 1992.

40. Yamada, J.; Sugimoto, T. (Eds.) TTF Chemistry: Fundamentals and Applications of Tetrathiafulvalene; Springer: Berlin/Heidelberg, Germany; New York, NY, USA, 2004.

41. Lebed, A. (Ed.) The Physics of Organic Superconductors and Conductors; Springer Series in Materials Science; Springe: Berlin, Germany, 2008; Volume 110.

42. Lin, C.Y.; George, M.W.; Gill, P.M.W. $\mathrm{EDF}_{2}$ : A Density Functional for Predicting Molecular Vibrational Frequencies. Aust. J. Chem. 2004, 57, 365-370.

43. Wavefunction, Inc. Spartan'14 for Windows, Maxintosh and Linux; Wavefunction, Inc.: Irvine, CA, USA, 2014.

44. Merrick, J.P.; Moran, D.; Radom, L. An Evaluation of Harmonic Vibrational Frequency Scale Factors. J. Phys. Chem. A 2007, 111, 11683-11700.

45. Ranzieri, P.; Masino, M.; Girlando, A. Charge-Sensitive Vibrations in $p$-Chloranil: The Strange Case of the C=C Antisymmetric Stretching. J. Phys. Chem. B 2007, 111, 12844-12848.

46. Liu, R.; Zhou, X.; Kasmai, H. Toward understanding the vibrational spectra of BEDT-TTF, a scaled density functional force field approach. Spectrochim. Acta A 1997, 53, 1241-1256.

47. Bozio, R.; Zanon, I.; Girlando, A.; Pecile, C. Vibrational spectroscopy of molecular constituents of one-dimensional organic conductors. Tetrathiofulvalene (TTF), $\mathrm{TTF}^{+}$, and $\left(\mathrm{TTF}^{+}\right)_{2}$ dimer. J. Chem. Phys. 1979, 71, 2282-2293.

48. Dressel, M.; Dumm, M.; Knoblauch, T.; Masino, M. Comprehensive Optical Investigations of Charge Order in Organic Chain Compounds (TMTTF) ${ }_{2}$ X. Crystals 2012, 2, 528-578.

49. Girlando, A.; Zanon, I.; Bozio, R.; Pecile, C. Raman and infrared frequency shifts proceeding from ionization of perhalo-p-benzoquinones to radical anions. J. Chem. Phys. 1978, 68, $22-31$.

50. Garcia, P.; Dahaoui, S.; Katan, C.; Souhassou, M.; Lecomte, C. On the accurate estimation of intermolecular interactions and charge transfer: The case of TTF-CA. Faraday Discuss. 2007, 135, 217-235.

51. Giannozzi, P.; Baroni, S.; Bonini, N.; Calandra, M.; Car, R.; Cavazzoni, C.; Ceresoli, D.; Chiarotti, G.L.; Cococcioni, M.; Dabo, I.; et al. QUANTUM ESPRESSO: A modular and open-source software project for quantum simulations of materials. J. Phys. Condens. Matter 2009, 21, 395502.

52. Perdew, J.P.; Burke, K.; Ernzerhof, M. Generalized Gradient Approximation Made Simple. Phys. Rev. Lett. 1996, 77, 3865-3868. 
53. Vanderbilt, D. Soft self-consistent pseudopotentials in a generalized eigenvalue formalism. Phys. Rev. B 1990, 41, 7892-7895.

54. Monkhorst, H.J.; Pack, J.D. Special points for Brillouin-zone integrations. Phys. Rev. B 1976, 13, 5188-5192.

55. Yu, P.Y.; Cardona, M. Fundamentals of Semiconductors: Physics and Materials Properties, 4th ed.; Graduate Texts in Physics; Springer: Berlin, Germany; London, UK, 2010.

56. Oison, V.; Katan, C.; Koenig, C. Intramolecular Electronic Redistribution Coupled to Hydrogen Bonding: An Important Mechanism for the "Neutral-to-Ionic" Transition. J. Phys. Chem. A 2001, 105, 4300-4307.

57. Dressel, M.; Drichko, N. Optical Properties of Two-Dimensional Organic Conductors: Signatures of Charge Ordering and Correlation Effects. Chem. Rev. 2004, 104, 5689-5716.

58. Tomic, S.; Dressel, M. Ferroelectricity in molecular solids: A review of electrodynamic properties. Rep. Prog. Phys. 2015, 78, 096501.

59. Girlando, A.; Pecile, C.; Brillante, A.; Syassen, K. High pressure optical studies of neutral-ionic phase transitions in organic charge-transfer crystals. Synth. Met. 1987, 19, 503-508.

60. Cointe, M.B.L.; Lemée-Cailleau, M.H.; Cailleau, H.; Toudic, B.; Moréac, A.; Moussa, F.; Ayache, C.; Karl, N. Thermal hysteresis phenomena and mesoscopic phase coexistence around the neutral-ionic phase transition in TTF-CA and TMB-TCNQ. Phys. Rev. B 2003, 68, 064103.

61. Ivek, T.; Korin-Hamzić, B.; Milat, O.; Tomić, S.; Clauss, C.; Drichko, N.; Schweitzer, D.; Dressel, M. Collective Excitations in the Charge-Ordered Phase of $\alpha$-(BEDT-TTF $)_{2} \mathbf{I}_{3}$. Phys. Rev. Lett. 2010, 104, 206406.

62. Ivek, T.; Korin-Hamzić, B.; Milat, O.; Tomić, S.; Clauss, C.; Drichko, N.; Schweitzer, D.; Dressel, M. Electrodynamic Response of the Charge Ordering Phase: Dielectric and Optical Studies of $\alpha$-(BEDT-TTF) ${ }_{2} \mathrm{I}_{3}$. Phys. Rev. B 2011, 83, 165128.

63. Alemany, P.; Pouget, J.P.; Canadell, E. Essential Role of Anions in the Charge Ordering Transition in $\alpha$-(BEDT-TTF) ${ }_{2} \mathrm{I}_{3}$. Phys. Rev. B 2012, 85, 195118.

64. Beyer, R.; Dengl, A.; Peterseim, T.; Wackerow, S.; Ivek, T.; Pronin, A.; Schweitzer, D.; Dressel, M. Pressure-dependent optical investigations of $\alpha$-(BEDT-TTF $)_{2} \mathrm{I}_{3}$ : Tuning charge order and narrow gap towards a Dirac semimetal. Phys. Rev. B 2016, 93, 195116.

65. Girlando, A.; Masino, M.; Painelli, A.; Drichko, N.; Dressel, M.; Brillante, A.; Della Valle, R.G.; Venuti, E. Direct evidence of overdamped Peierls-coupled modes in the temperature-induced phase transition in tetrathiafulvalene-chloranil. Phys. Rev. B 2008, 78, 045103.

66. Tadashi, S.; Kyuya, Y.; Haruo, K. Polarized Absorption Spectra of Single Crystals of Tetrathiafulvalenium Salts. Bull. Chem. Soc. Jpn. 1978, 51, 1041-1046.

67. Masashi, T. Electronic States of the Crystals of TCNE Complexes with Hexamethylbenzene, Acenaphthene, and Dibenzofuran. Bull. Chem. Soc. Jpn. 1977, 50, 2881-2884.

68. Masino, M.; Girlando, A.; Soos, Z.G. Evidence for a soft mode in the temperature induced neutral-ionic transition of TTF-CA. Chem. Phys. Lett. 2003, 369, 428-433.

69. Koshihara, S.; Tokura, Y.; Mitani, T.; Saito, G.; Koda, T. Photoinduced valence instability in the organic molecular compound tetrathiafulvalene-p-chloranil (TTF-CA). Phys. Rev. B 1990, 42, 6853-6856.

70. Koshihara, S.; Takahashi, Y.; Sakai, H.; Tokura, Y.; Luty, T. Photoinduced Cooperative Charge Transfer in Low-Dimensional Organic Crystals. J. Phys. Chem. B 1999, 103, 2592-2600.

71. Huai, P.; Zheng, H.; Nasu, K. Theory for Photoinduced Ionic-Neutral Structural Phase Transition in Quasi One-Dimensional Organic Molecular Crystal TTF-CA. J. Phys. Soc. Jpn. 2000, 69, 1788-1800.

72. Collet, E.; Lemée-Cailleau, M.H.; Cointe, M.B.L.; Cailleau, H.; Ravy, S.; Luty, T.; Bérar, J.F.; Czarnecki, P.; Karl, N. Direct evidence of lattice-relaxed charge transfer exciton strings. Europhys. Lett. 2002, 57, 67-73.

73. Iwai, S.; Tanaka, S.; Fujinuma, K.; Kishida, H.; Okamoto, H.; Tokura, Y. Ultrafast Optical Switching from an Ionic to a Neutral State in Tetrathiafulvalene- $p$-Chloranil (TTF-CA) Observed in Femtosecond Reflection Spectroscopy. Phys. Rev. Lett. 2002, 88, 057402.

74. Nasu, K. (Ed.) Photoinduced Phase Transition; Word Scientific: Singapore, 2004.

75. Tanimura, K. Femtosecond time-resolved reflection spectroscopy of photoinduced ionic-neutral phase transition in TTF-CA crystals. Phys. Rev. B 2004, 70, 144112.

76. Okamoto, H.; Ishige, Y.; Tanaka, S.; Kishida, H.; Iwai, S.; Tokura, Y. Photoinduced phase transition in tetrathiafulvalene- $p$-chloranil observed in femtosecond reflection spectroscopy. Phys. Rev. B 2004, 70, 165202. 
77. Guérin, L.; Collet, E.; Lemée-Cailleau, M.H.; Cointe, M.B.L.; Cailleau, H.; Plech, A.; Wulff, M.; Koshihara, S.Y.; Luty, T. Probing photoinduced phase transition in a charge-transfer molecular crystal by 100 picosecond X-ray diffraction. Chem. Phys. 2004, 299, 163-170.

78. Collet, E.; Cointe, M.B.L.; Cailleau, H. X-ray Diffraction Investigation of the Nature and the Mechanism of Photoinduced Phase Transition in Molecular Materials. J. Phys. Soc. Jpn. 2006, 75, 011002.

79. Tokura, Y. Photoinduced Phase Transition: A Tool for Generating a Hidden State of Matter. J. Phys. Soc. Jpn. 2006, 75, 011001.

80. Yonemitsu, K. Theory of Photoinduced Phase Transitions in Molecular Conductors: Interplay between Correlated Electrons, Lattice Phonons and Molecular Vibrations. Crystals 2012, 2, 56-77.

81. Iwai, S.; Okamoto, H. Ultrafast Phase Control in One-Dimensional Correlated Electron Systems. J. Phys. Soc. Jpn. 2006, 75, 011007.

82. Matsubara, Y.; Okimoto, Y.; Yoshida, T.; Ishikawa, T.; Koshihara, S.Y.; Onda, K. Photoinduced Neutral-to-Ionic Phase Transition in Tetrathiafulvalene- $p$-chloranil Studied by Time-Resolved Vibrational Spectroscopy. J. Phys. Soc. Jpn. 2011, 80, 124711.

83. Peterseim, T.; Haremski, P.; Dressel, M. Random-walk annihilation process of photo-induced neutral-ionic domain walls in TTF-CA. Europhys. Lett. 2015, 109, 67003.

84. Peterseim, T.; Dressel, M. Molecular Dynamics at Electrical- and Optical-Driven Phase Transitions: Time-Resolved Infrared Studies Using Fourier-Transform Spectrometers. arXiv 2016, arXiv:1604.00977.

85. Suzuki, T.; Sakamaki, T.; Tanimura, K.; Koshihara, S.; Tokura, Y. Ionic-to-neutral phase transformation induced by photoexcitation of the charge-transfer band in tetrathiafulvalene-p-chloranil crystals. Phys. Rev. B 1999, 60, 6191-6193.

86. Tanimura, K.; Koshihara, S. Photo-induced ionic-to-neutral phase transition in tetrathiafulvalen- $p$-chloranil crystals. Phase Transit. 2001, 74, 21-34.

87. Iwai, S.; Ishige, Y.; Tanaka, S.; Okimoto, Y.; Tokura, Y.; Okamoto, H. Coherent Control of Charge and Lattice Dynamics in a Photoinduced Neutral-to-Ionic Transition of a Charge-Transfer Compound. Phys. Rev. Lett. 2006, 96, 057403.

88. Tanimura, K.; Akimoto, I. Femtosecond time-resolved spectroscopy of photoinduced ionic-to-neutral phase transition in tetrathiafulvalen-p-chloranil crystals. J. Lumin. 2001, 94-95, 483-488.

89. Tokura, Y.; Koda, T.; Mitani, T.; Saito, G. Neutral-to-ionic transition in tetrathiafulvalene- $p$-chloranil as investigated by optical reflection spectra. Solid State Commun. 1982, 43, 757-760.

90. Hünig, S.; Kießlich, G.; Quast, H.; Scheutzow, D. Über zweistufige Redoxsysteme, $X^{1}$ Tetrathio-äthylene und ihre höheren Oxidationsstufen. Justus Liebigs Ann. Chem. 1973, 1973, 310-323.

91. Wudl, F.; Kruger, A.A.; Kaplan, M.L.; Hutton, R.S. Unsymmetrical dimethyltetrathiafulvalene. J. Org. Chem. 1977, 42, 768-770.

92. Coffen, D.L.; Chambers, J.Q.; Williams, D.R.; Garrett, P.E.; Canfield, N.D. Tetrathioethylenes. J. Am. Chem. Soc. 1971, 93, 2258-2268.

93. Andre, J.; Weill, G. Optical spectrum of the chloranil radical anion. Mol. Phys. 1968, 15, 97-99.

94. Nobuko, S.; Ichimin, S.; Shigeru, M. The Effect of Pressure on the Electronic Absorption Spectra of Alkali Metal Cation-Chloranil and -Bromanil Anion Radical Salts, and the Dimerization of the Chloranil Anion Radical in Solution. Bull. Chem. Soc. Jpn. 1971, 44, 675-679.

95. Bieber, A.; Andre, J. Some electronic properties of TMPD, CA and TCNQ molecules and their mono- and diions. Chem. Phys. 1974, 5, 166-182.

96. Pope, M.; Swenberg, C.E. Electronic Processes in Organic Crystals and Polymers, 2nd ed.; Monographs on the Physics and Chemistry of Materials; Oxford University Press: New York, NY, USA, 1999; Volume 56.

97. Hoshino, M.; Nozawa, S.; Sato, T.; Tomita, A.; Adachi, S.I.; Koshihara, S.Y. Time-resolved X-ray crystal structure analysis for elucidating the hidden 'over-neutralized' phase of TTF-CA. RSC Adv. 2013, 3, 16313-16317.

98. Dressel, M.; Grüner, G. Electrodynamics of Solids; Cambridge University Press: Cambridge, UK, 2002.

99. Nagahori, A.; Kubota, N.; Itoh, C. Time-resolved Fourier-transform infrared reflection study on photoinduced phase transition of tetrathiafulvalene-p-chloranil crystal. Eur. Phys. J. B 2013, 86, 109-142.

100. Djurek, D.; Jerome, D.; Bechgaard, K. Thermal transport properties of organic conductors: $\left(\mathrm{TMTSF}_{2} \mathrm{PF}_{6}\right.$ and (TMTSF $)_{2} \mathrm{ClO}_{4}$. J. Phys. C Solid State Phys. 1984, 17, 4179-4192.

101. Bechtel, J.H. Heating of solid targets with laser pulses. J. Appl. Phys. 1975, 46, 1585-1593. 
102. Salamon, M.B.; Bray, J.W.; DePasquali, G.; Craven, R.A.; Stucky, G.; Schultz, A. Thermal conductivity of tetrathiafulvalene-tetracyanoquinodimethane (TTF-TCNQ) near the metal-insulator transition. Phys. Rev. B 1975, 11, 619-622.

103. Matsukawa, M.; Hashimoto, K.; Yoshimoto, N.; Yoshizawa, M.; Kashiwaba, Y.; Noto, K. Thermal Conductivity in the $a b$-Plane of the Organic Conductor $\alpha$-BEDT-TTF) $)_{2} \mathrm{I}_{3}$. J. Phys. Soc. Jpn. 1995, 64, 2233-2234.

104. Nasu, K. Relaxations of Excited States and Photo-Induced Structural Phase Transitions; Springer Series in Solid-State Sciences; Springer: Berlin/Heidelberg, Germany, 1997; Volume 124.

105. Kuroda, N.; Wakabayashi, Y.; Nishida, M.; Wakabayashi, N.; Yamashita, M.; Matsushita, N. Decay Kinetics of Long-Lived Photogenerated Kinks in an MX Chain Compound. Phys. Rev. Lett. 1997, 79, 2510-2513.

106. Tabata, Y.; Kuroda, N. Decay Kinetics of Photoinduced Solitons on a One-Dimensional Lattice with Traps. J. Phys. Soc. Jpn. 2009, 78, 034704.

107. Koshihara, S.; Tokura, Y.; Sarukura, N.; Segawa, Y.; Koda, T.; Takeda, K. Nanosecond and picosecond dynamics of photo-induced phase-transition in low dimensional organic crystals. Synth. Met. 1995, 70, 1225-1226.

108. Bleier, H.; Roth, S.; Shen, Y.Q.; Schäfer-Siebert, D.; Leising, G. Photoconductivity in trans-polyacetylene: Transport and recombination of photogenerated charged excitations. Phys. Rev. B 1988, 38, 6031-6040.

109. Shank, C.V.; Yen, R.; Fork, R.L.; Orenstein, J.; Baker, G.L. Picosecond Dynamics of Photoexcited Gap States in Polyacetylene. Phys. Rev. Lett. 1982, 49, 1660-1663.

110. Privman, V. Nonequilibrium Statistical Mechanics in One Dimension; Cambridge University Press: Cambridge, UK; New York, NY, USA, 1997.

111. Torney, D.C.; McConnell, H.M. Diffusion-limited reactions in one dimension. J. Phys. Chem. 1983, 87, 1941-1951.

112. Lushnikov, A. Binary reaction $1+1 \rightarrow 0$ in one dimension. Phys. Lett. A 1987, 120, 135-137.

113. Balding, D.; Clifford, P.; Green, N. Invasion processes and binary annihilation in one dimension. Phys. Lett. A 1988, 126, 481-483.

114. Sasaki, K.; Nakagawa, T. Exact Results for a Diffusion-Limited Pair Annihilation Process on a One-Dimensional Lattice. J. Phys. Soc. Jpn. 2000, 69, 1341-1351.

115. Ben Avraham, D. Computer simulation methods for diffusion-controlled reactions. J. Chem. Phys. 1988, 88, 941-948.

116. bwGRiD, Member of the German D-Grid Initiative, Funded by the Ministry for Education and Research (Bundesministerium für Bildung und Forschung) and the Ministry for Science, Research and Arts Baden-Wuerttemberg (Ministerium für Wissenschaft, Forschung und Kunst Baden-Württemberg). Available online: http:/ / www.bw-grid.de (accessed on 4 January 2017).

117. Fano, U. Effects of Configuration Interaction on Intensities and Phase Shifts. Phys. Rev. 1961, 124, $1866-1878$.

118. Sedlmeier, K.; Elsässer, S.; Neubauer, D.; Beyer, R.; Wu, D.; Ivek, T.; Tomić, S.; Schlueter, J.A.; Dressel, M. Absence of charge order in the dimerized $\kappa$-phase BEDT-TTF salts. Phys. Rev. B 2012, 86, 245103.

119. Gordon, M.S.; Schmidt, M.W. Advances in electronic structure theory: \{GAMESS\} a decade later. In Theory and Applications of Computational Chemistry; Dykstra, C.E., Frenking, G., Kim, K.S., Scuseria, G.E., Eds.; Elsevier: Amsterdam, The Netherlands, 2005; Chapter 41, pp. 1167-1189.

120. Schmidt, M.W.; Baldridge, K.K.; Boatz, J.A.; Elbert, S.T.; Gordon, M.S.; Jensen, J.H.; Koseki, S.; Matsunaga, N.; Nguyen, K.A.; Su, S.; et al. General atomic and molecular electronic structure system. J. Comput. Chem. 1993, 14, 1347-1363.

121. Perdew, J.P.; Schmidt, K. Jacob's ladder of density functional approximations for the exchange-correlation energy. In Proceedings of the Conference on Density Functional Theory and Its Application To Materials, Antwep, Belgium, 8-10 June 2000.

122. Becke, A.D. Density-functional exchange-energy approximation with correct asymptotic behavior. Phys. Rev. A 1988, 38, 3098-3100.

123. Lee, C.; Yang, W.; Parr, R.G. Development of the Colle-Salvetti correlation-energy formula into a functional of the electron density. Phys. Rev. B 1988, 37, 785-789.

124. Perdew, J.P.; Wang, Y. Accurate and simple analytic representation of the electron-gas correlation energy. Phys. Rev. B 1992, 45, 13244-13249.

125. Perdew, J.P.; Burke, K.; Ernzerhof, M. Generalized Gradient Approximation Made Simple. Phys. Rev. Lett. $1997,78,1396$. 
126. Becke, A.D. Density-functional thermochemistry. III. The role of exact exchange. J. Chem. Phys. 1993, 98, 5648-5652.

127. Becke, A.D. A new mixing of Hartree-Fock and local density-functionalq theories. J. Chem. Phys. 1993, 98, 1372-1377.

128. Stephens, P.J.; Devlin, F.J.; Chabalowski, C.F.; Frisch, M.J. Ab Initio Calculation of Vibrational Absorption and Circular Dichroism Spectra Using Density Functional Force Fields. J. Phys. Chem. 1994, 98, 11623-11627.

129. Adamo, C.; Barone, V. Toward reliable density functional methods without adjustable parameters: The PBE0 model. J. Chem. Phys. 1999, 110, 6158-6170.

130. Jensen, F. Introduction to Computational Chemistry, 2nd ed.; John Wiley \& Sons: Chichester, UK; Hoboken, NJ, USA, 2007.

131. Andersson, M.P.; Uvdal, P. New Scale Factors for Harmonic Vibrational Frequencies Using the B3LYP Density Functional Method with the Triple- $\zeta$ Basis Set 6-311+G(d,p). J. Phys. Chem. A 2005, 109, 2937-2941.

132. Carbonniere, P.; Lucca, T.; Pouchan, C.; Rega, N.; Barone, V. Vibrational computations beyond the harmonic approximation: Performances of the B3LYP density functional for semirigid molecules. J. Comput. Chem. 2005, 26, 384-388.

133. Gisslén, L.; Scholz, R. Crystallochromy of perylene pigments: Interference between Frenkel excitons and charge-transfer states. Phys. Rev. B 2009, 80, 115309.

134. Kokalj, A. XCrySDen-A new program for displaying crystalline structures and electron densities. J. Mol. Graph. Model. 1999, 17, 176-179.

135. Benassi, A. PWscf's Epsilon.x User's Manual. Available online: https://www.google.de/url?sa=t\&rct=j\&q= \&esrc=s\&source=web\&cd=1\&cad=rja\&uact=8\&ved=0ahUKEwj15MbqqanRAhWFwBQKHWOMA_ 4QFggaMAA\&url=https\%3A\%2F\%2Fwww.researchgate.net\%2Ffile.PostFileLoader.html\%3Fid\% 3D567d1ab17eddd3eb328b4574\%26assetKey\%3DAS\%253A310495943299072\%25401451039409313\& usg=AFQjCNGjbPOaQq47wPrCah_71TeU8DCUpw\&sig2=D8sZeomhzyoi9HayKAfzDw (accessed on 30 November 2016).

(C) 2017 by the authors; licensee MDPI, Basel, Switzerland. This article is an open access article distributed under the terms and conditions of the Creative Commons Attribution (CC-BY) license (http://creativecommons.org/licenses/by/4.0/). 